\title{
A Gross-Kohnen-Zagier type theorem for higher-codimensional Heegner cycles
}

\author{
Shaul Zemel
}

\section{Correspondence:}

zemels@math.huji.ac.il

Einstein Institute of Mathematics, the Hebrew University of Jerusalem, Edmund Safra Campus, Jerusalem 91904, Israel

\begin{abstract}
We prove that the Heegner cycles of codimension $m+1$ inside Kuga-Sato type varieties of dimension $2 m+1$ are coefficients of modular forms of weight $3 / 2+m$ in the appropriate quotient group. The main technical tool for generating the necessary relations is a Borcherds style theta lift with polynomials. We also show how this lift defines a new singular Shimura-type correspondence from weakly holomorphic modular forms of weight $1 / 2-m$ to meromorphic modular forms of weight $2 m+2$.
\end{abstract}

\section{Introduction}

A celebrated theorem of Gross, Kohnen, and Zagier in [15] states that the Heegner divisors on modular curves correspond, in parts of the Jacobian variety of the modular curve, to coefficients of a modular form of weight $\frac{3}{2}$. This result was proved using height evaluations. Borcherds gave in [3] another proof of this theorem, using his singular theta lift from [2]. The latter proof generalizes to Shimura curves, and the argument extends to yield the modularity of Hirzebruch-Zagier divisors from [16] on Hilbert modular surfaces, of Humbert surfaces on Siegel threefolds, etc. The goal of this paper is to establish a modularity result for cycles of higher codimension inside the universal families arising from the moduli interpretation of Shimura and modular curves.

Shimura curves parametrize Abelian surfaces with quaternionic multiplication. This implies (under some technical assumptions) the existence of universal families of Abelian surfaces over these curves. By taking the $m$ th symmetric power, we obtain a KugaSato type variety, of dimension $2 m+1$, in which the fiber over a point in the Shimura curve is the product of $m$ copies of the Abelian surface it represents. In correspondence with universal families of elliptic curves, we denote this variety $W_{2 m}$. The CM points on the Shimura curve correspond to those Abelian surfaces whose endomorphism ring, or equivalently whose group of cycles, is larger than the generic one. The $m$ th power of such an additional, normalized cycle has dimension $m$, and considering it as a subvariety of the fiber of $W_{2 m}$, it has codimension $m+1$ in the latter variety. One defines the Heegner cycles to be certain combinations of these cycles in $W_{2 m}$. As these cycles are cohomologically trivial, one would like to investigate their images in the intermediate Jacobian of $W_{2 m}$, or in Hodge components of this Jacobian. There are several results indicating the modularity of related objects in related groups (see, e.g., [31] or [17]). This paper is proving another result of this type, namely

(c) 2015 Zemel. Open Access This article is distributed under the terms of the Creative Commons Attribution 4.0 International License (http://creativecommons.org/licenses/by/4.0/), which permits unrestricted use, distribution, and reproduction in any medium, provided you give appropriate credit to the original author(s) and the source, provide a link to the Creative Commons license, and indicate if changes were made. 
Theorem. The images of the $(m+1)$-codimensional Heegner cycles in $W_{2 m}$ correspond, in an appropriate quotient group, to the coefficients of a modular form of weight $\frac{3}{2}+m$.

Before discussing the method of proof, we mention that the CM cycles are defined in the modular case as actual cycles (normalized graphs of CM isogenies), but in the general case only cohomology classes are considered (see, e.g., Section 5 of [1]). A first aim of this paper is to describe the CM cycles also in the non-split case as actual CM cycles inside the Abelian surface (see Definition 1.16). We then evaluate the fundamental cohomology classes of these cycles explicitly, as well as simplify the proofs of several assertions from [1], using de-Rham cohomology. These CM cycles form, together with the generic cycles in case the quaternion algebra is trivial, all the Abelian subvarieties of Abelian surfaces with QM. However, the main result is proved for the corresponding cohomology classes, or Hodge vectors in variations of Hodge structures.

Our method of proof follows [3] closely, but using the more general theta lift from [2], allowing the theta kernel of the even lattice $L$ of signature $\left(2, b_{-}\right)$to involve certain homogenous polynomials on $L_{\mathbb{R}}$. The resulting functions are not meromorphic on the complex manifold $G\left(L_{\mathbb{R}}\right)$, but are eigenfunctions of eigenvalue $-2 m b_{-}$with respect to the weight $m$ Laplacian on $G\left(L_{\mathbb{R}}\right)$, with known singularities. In the case $b_{-}=1$ of Shimura and modular curves, the images of these theta lifts under the weight raising operators are meromorphic (with known poles). This establishes a new singular Shimura-type correspondence, as stated in the following

Theorem. Let $m$ be an even positive integer and let $f=\sum_{n} c_{n} q^{n}$ be a weakly holomorphic modular form of weight $\frac{1}{2}-m$ with respect to $\Gamma_{0}(4)$ which lies in the Kohnen plus-space. Then the function

$$
\frac{i^{m}}{2} \delta_{2 m} \Phi_{L, m, m, 0}(v, F)=\sum_{r=1}^{\infty}\left(\sum_{d \mid r} d^{m+1} c_{d^{2}}\right) r^{m} q^{r}
$$

is a meromorphic modular form of weight $2 m+2$ with respect to $S L_{2}(\mathbb{Z})$. Its poles are all of order $m+1$, and they lie at CM points on the upper half-plane. More explicitly, $\frac{i^{m}}{2} \delta_{2 m} \Phi_{L, m, m, 0}(v, F)$ has a pole at a CM point of negative discriminant $-D$ if and only if the Fourier coefficient $c_{-D}$ off does not vanish.

The method is developed here in a way aiming for proving similar results also for higher-dimensional base spaces. Indeed, the subsequent work investigates the results thus obtained for the case $b_{-}=2$. It requires a non-trivial analysis of the theta lift, which is based on the machinery from [2], but has to be examined explicitly since these particular functions do not appear in that reference. The differential properties are established by arguments similar to those appearing in [6]. Additional useful properties of our theta lifts are studied in [29].

This paper is divided into 4 sections. In Section 1 we discuss universal families over Shimura curves and the corresponding variations of Hodge structures, as well as the CM cycles and their properties. In Section 2 we derive a differential equation which is satisfied by Siegel theta functions. Section 3 presents the singular theta lift which we are using, as well as its meromorphic image in the case $b_{-}=1$ (the singular Shimura-type correspondence stated above). Finally, Section 4 defines the relations which we consider between 
the $\mathrm{CM}$ (or Heegner) cycles in question, proves the main result of this paper, and suggests possible connections between our results and existing theorems and conjectures.

I am deeply indebted to my Ph.D. advisor R. Livné, for proposing the questions discussed here, for many enlightening discussions, and for being a constant source of support during the work on this project. I would also like to thank H. Farkas for his help during that time. Many thanks are due to J. Bruinier for numerous comments and suggestions, which greatly improved this paper relative to the original version. The help of S. Ehlen in the evaluation and verification of several explicit examples of the singular Shimuratype lift is also acknowledged. My special thanks are delivered to the anonymous referee, whose suggestions greatly improved the readability of this paper.

\section{CM Cycles in universal families}

In this Section we present universal families of Abelian surfaces over Shimura (and modular) curves, and the variations of Hodge structures arising from them. We give the definition of general CM cycles inside such surfaces having also CM, and show how to evaluate their fundamental cohomology classes.

\subsection{Variations of Hodge structures over Shimura curves}

Let $\mathcal{H}$ denote the upper half plane $\{\tau=x+i y \in \mathbb{C} \mid \Im \tau>0\}$. The Shimura variation of Hodge structure over $\mathcal{H}$, denoted $V_{1}$, is defined (see also Section 5 of [1] and Section 3 of [10]) as follows. The real local system is just $\mathcal{H} \times \mathbb{R}^{2}$. Tensoring the fiber over $\tau \in \mathcal{H}$ with $\mathbb{C}$, we fix $\left(\begin{array}{l}\tau \\ 1\end{array}\right)$ to have Hodge weight $(1,0)$ and $\left(\begin{array}{l}\bar{\tau} \\ 1\end{array}\right)$ to have Hodge weight $(0,1)$ there. The determinant of $2 \times 2$ matrices defines a map $\mathbb{R}^{2} \times \mathbb{R}^{2} \rightarrow \mathbb{R},(u, v) \mapsto \operatorname{det}((u, v))$, which polarizes $V_{1}$. Let $V_{m}$ be be the $m$ th symmetric power of $V_{1}$, elements of whose fibers we write as homogenous polynomials of degree $m$ in the fiber of $V_{1}$. Then the vector $\left(\begin{array}{l}\tau \\ 1\end{array}\right)^{m_{+}}\left(\begin{array}{l}\bar{\tau} \\ 1\end{array}\right)^{m_{-}}$, with $m_{+}+m_{-}=m$, has Hodge type $\left(m_{+}, m_{-}\right)$in the complexification of the fiber of $V_{m}$ over $\tau \in \mathcal{H}$.

Given $\tau \in \mathcal{H}$, we define

$$
M_{\tau}=\left(\begin{array}{c}
\tau-\tau^{2} \\
1-\tau
\end{array}\right), \quad J_{\tau}=\frac{1}{y}\left(\begin{array}{cc}
x & -|\tau|^{2} \\
1 & -x
\end{array}\right), \quad \text { and } \quad M_{\bar{\tau}}=\left(\begin{array}{c}
\bar{\tau}-\bar{\tau}^{2} \\
1-\bar{\tau}
\end{array}\right) .
$$

Recall that the action of $A=\left(\begin{array}{ll}a & b \\ c & d\end{array}\right) \in G L_{2}^{+}(\mathbb{R})$ sends $\tau \in \mathcal{H}$ to $A \tau=\frac{a \tau+b}{c \tau+d}$, with automorphy factor $j(A, \tau)=c \tau+d$. We have the useful equation

$$
A\left(\begin{array}{l}
\tau \\
1
\end{array}\right)=j(A, \tau)\left(\begin{array}{c}
A \tau \\
1
\end{array}\right)
$$

as well as the relations

$$
A M_{\tau} \operatorname{adj} A=j(A, \tau)^{2} M_{A \tau} \text { and } A J_{\tau} A^{-1}=J_{A \tau} .
$$

Here $\operatorname{adj} A=\operatorname{det} A \cdot A^{-1}=\left(\begin{array}{cc}d & -b \\ -c & a\end{array}\right)$ for $A$ as above. More generally, Eq. (1) holds for every $A \in M_{2}(\mathbb{R})$ and $\tau \in \mathbb{C}$ for which the natural extension of $j(A, \tau)$ does not vanish (so that $A \tau$ makes sense). In addition, Eq. (2) extends to the case of $A \in G L_{2}(\mathbb{R})$ and $\tau \in \mathcal{H} \cup \overline{\mathcal{H}}$ (where $J_{\bar{\tau}}=-J_{\tau}$ ). We have

\section{Proposition 1.1. The following assertions are true:}

(i) Multiplication of $\left(\begin{array}{l}\tau \\ 1\end{array}\right)$ by any non-zero complex number can be achieved by the action of $G L_{2}^{+}(\mathbb{R})$. 
(ii) $V_{1}$, hence also $V_{m}$ for every $m$, is $G L_{2}^{+}(\mathbb{R})$-equivariant. $S L_{2}(\mathbb{R})$ preserves the polarization as well. Hence they descent to variations of Hodge structures on quotients of $\mathcal{H}$ by Fuchsian groups.

Proof. Part ( $i$ ) follows by Eq. (1) from the fact that $M=d I+c J_{\tau}$ satisfies $M \tau=\tau$ and $j(M, \tau)=c i+d$. Part (ii) is an immediate consequence of Eq. (1). This proves the proposition.

Let $B$ be a rational quaternion algebra, i.e., a 4-dimensional central simple algebra over $\mathbb{Q}$, with reduced trace $\operatorname{Tr}: B \rightarrow \mathbb{Q}$, reduced norm $N: B^{\times} \rightarrow \mathbb{Q}^{\times}$, and main involution $x \mapsto \bar{x}$. For background on (rational) quaternion algebras we refer the reader to [27], for example. Assume that $B$ splits over $\mathbb{R}$, and fix an isomorphism $i: B_{\mathbb{R}} \stackrel{\sim}{\rightarrow} M_{2}(\mathbb{R})$. We identify $B_{\mathbb{R}}$ with $M_{2}(\mathbb{R})$ via $i$, hence omit $i$ from the notation. Recall that an ideal in a rational quaternion algebra is a finitely generated subgroup of full rank in $B$, and an order is an ideal which is a subring of $B$. An order is maximal if it is not contained in any larger order in $B$, and is Eichler if it is the intersection of two maximal orders. Given an order $\mathcal{M}$ in $B$, we consider Abelian surfaces $A$ with quaternionic multiplication (QM) from $\mathcal{M}$. To be precise, the latter statement means not only that $\mathcal{M}$ is embedded into $A$, but also that the intersection of $\operatorname{End}(A)$ with $B \subseteq \operatorname{End}(A)_{\mathbb{Q}}$ is $\mathcal{M}$ (i.e., if $\widetilde{\mathcal{M}}$ is a larger order in $B$ which is embedded into $\operatorname{End}(A)$ we say that $A$ has QM from $\widetilde{\mathcal{M}}$, and not from $\mathcal{M})$. As an example of such a surface, take $\tau \in \mathcal{H} \cup \overline{\mathcal{H}}$ and an ideal $I \subseteq B$ such that the order $L(I)=\{x \in B \mid x I \subseteq I\}$ is equal to $\mathcal{M}$, and define $A_{\tau}=\mathbb{C}^{2} / I\left(\begin{array}{l}\tau \\ 1\end{array}\right) . \mathcal{M}$ embeds into $\operatorname{End}\left(A_{\tau}\right)$ by identifying $\mathbb{C}^{2}$ with $M_{2}(\mathbb{R})\left(\begin{array}{l}\tau \\ 1\end{array}\right)$ and letting $\mathcal{M}$ operate on $I$ and $M_{2}(\mathbb{R})$. The Shimura curve associated with $I$ is the quotient of $\mathcal{H}$ by the group $R(I)_{+}^{\times}$of (invertible) elements of reduced norm 1 in the order $R(I)=\{x \in B \mid I x \subseteq I\}$. We now have

Lemma 1.2. Let $\tau$ and $\sigma$ be elements of $\mathcal{H} \cup \overline{\mathcal{H}}$, and let I and J be ideals in B such that $L(I)=L(J)=\mathcal{M}$. Then the maps $\mathbb{C}^{2} / J\left(\begin{array}{l}\sigma \\ 1\end{array}\right) \rightarrow \mathbb{C}^{2} / I\left(\begin{array}{l}\tau \\ 1\end{array}\right)$ which commute with the action of $\mathcal{M}$ are in one-to-one correspondence with elements $M \in B$ satisfying $M \tau=\sigma$ and $J M \subseteq I$. Every such non-zero map is an isogeny, and it is an isomorphism if and only if JM $=I$.

Proof. Any map between these two Abelian surfaces gives a linear map from $\mathbb{C}^{2}=$ $M_{2}(\mathbb{R})\left(\begin{array}{l}\sigma \\ 1\end{array}\right)$ to $\mathbb{C}^{2}=M_{2}(\mathbb{R})\left(\begin{array}{l}\tau \\ 1\end{array}\right)$ between the tangent spaces. Commutation with the (left) action of $\mathcal{M}$ means that this map is described by right multiplication on $M_{2}(\mathbb{R})$ by some matrix $M$. Considering the $H_{1}$ groups shows that $M$ must satisfy $J M \subseteq I$. In particular $M \in B$. On the complex vector spaces, the only maps which commute with $\mathcal{M}$ are multiplication by scalars, which yields the equality $M\left(\begin{array}{l}\tau \\ 1\end{array}\right)=j\left(\begin{array}{l}\sigma \\ 1\end{array}\right)$. If $j=0$ then $M=0$ and we are done. Otherwise Eq. (1) yields $\sigma=M \tau$ (and $j=j(M, \tau)$ ). This implies $M \in B^{\times}$(for $M \neq 0)$, so that a certain integral multiple of $M^{-1}$ yields a map in the other direction. As one may use $M^{-1}$ itself for the map $\mathbb{C}^{2} / I\left(\begin{array}{l}\tau \\ 1\end{array}\right) \rightarrow \mathbb{C}^{2} / J\left(\begin{array}{l}\sigma \\ 1\end{array}\right)$ if and only if $J M=I$, this completes the proof of the lemma.

The geometric interpretation of Shimura curves is given in

Proposition 1.3. The moduli space of Abelian surfaces having $Q M$ from $\mathcal{M}$ is a finite disjoint union of Shimura curves. If $\mathcal{M}$ is an Eichler order, this space is a single Shimura curve. 
Proof. As a real manifold, an Abelian surface $A$ with QM from $\mathcal{M}$ is $V / \Lambda$ with $\Lambda=$ $H_{1}(A, \mathbb{Z})$ and $V=T_{0}(A)=\Lambda_{\mathbb{R}}$. Both $\Lambda$ and $V$ are $\mathcal{M}$-modules. As $B$ has exactly one module of every $\mathbb{Q}$-dimension divisible by $4, \Lambda_{\mathbb{Q}}$ may be identified with $B$ (hence $V$ with $M_{2}(\mathbb{R})$ ), and $\Lambda$ with some ideal $I \subseteq B$ such that $L(I)=\mathcal{M}$. The complex structure commutes with the action of $\mathcal{M}$, hence is defined through right multiplication by a matrix in $M_{2}(\mathbb{R})$. This matrix must thus be of the form $J_{\tau}$ for some $\tau \in \mathcal{H} \cup \overline{\mathcal{H}}$. Identifying elements of the holomorphic tangent space of $A$ with their first column multiplied by $i y$ shows that $A$ is isomorphic to $A_{\tau}$ defined above. Now, Lemma 1.2 yields an isomorphism $\mathbb{C}^{2} / I M^{-1}\left(\begin{array}{c}M \tau \\ 1\end{array}\right) \rightarrow \mathbb{C}^{2} / I\left(\begin{array}{l}\tau \\ 1\end{array}\right)$ for any $M \in B^{\times}$. Applying this to $M$ with $N(M)<0$ shows that every $A_{\tau}$ with $\tau \in \overline{\mathcal{H}}$ is isomorphic to some $A_{\sigma}$ with $\sigma \in \mathcal{H}$ (and perhaps a different ideal). Hence we may restrict attention to indices from $\mathcal{H}$ alone. The same argument shows that we may also assume that the ideal $I$ belongs to a set of representatives for the classes of ideals $I$ with $L(I)=\mathcal{M}$ modulo right multiplication from $B^{\times}$. There are finitely many such classes, and if $\mathcal{M}$ is an Eichler order then there is only one such class (see [27]). In addition, for fixed $I$, Lemma 1.2 shows that if $\tau$ and $\sigma$ are in $\mathcal{H}$ then $A_{\tau} \cong A_{\sigma}$ if and only if $\sigma=M \tau$ for $M \in R(I)^{\times}$. Such $M$ must also have positive reduced norm since $\tau$ and $M \tau$ are both in $\mathcal{H}$. This proves the proposition.

Let $\pi: \mathcal{A} \rightarrow \mathcal{H}$ be the universal Abelian surface with $\mathrm{QM}$ from the order $\mathcal{M}$ (say with ideal $I$ ) as in, e.g., Section 4 of [1]. This means that the fiber $\pi^{-1}(\tau)$ is $A_{\tau}$. Let $\Gamma \subseteq R(I)_{+}^{\times}$ be of finite index, and let $X(\Gamma)$ be the associated Shimura curve. This is just $\Gamma \backslash \mathcal{H}$ if $B$ is not split. The map in which $M \in \Gamma$ takes $v+\left.I\left(\begin{array}{c}\tau \\ 1\end{array}\right)\right|_{\tau}\left(\right.$ with $\left.v \in \mathbb{C}^{2}\right)$ to $\frac{v}{j(M, \tau)}+\left.I\left(\begin{array}{c}M \tau \\ 1\end{array}\right)\right|_{M \tau}$ is a well-defined action of $\Gamma$ on $\mathcal{A}$ over $\mathcal{H}$ (see the proof of Lemma 1.2), yielding a universal Abelian surface with QM over $X(\Gamma)$ (at least when $\Gamma$ has no elements with fixed points in $\mathcal{H})$, as well as variations of Hodge structures on $X(\Gamma)$, which we describe below. As such a universal family is the quotient of $\mathcal{H} \times \mathbb{C}^{2}$ by the semi-direct product of $\Gamma$ and $I$, this suggests the existence of a theory of Jacobi-like forms on $\mathcal{H} \times \mathbb{C}^{2}$ in which $\Gamma$ is the group acting on $\mathcal{H}$.

Let $I$ be an ideal in $B$, with discriminant $\operatorname{disc}(I)$, and consider the corresponding Abelian surface $A_{\tau}$ with $\tau \in \mathcal{H}$. As $H_{1}\left(A_{\tau}, \mathbb{Z}\right)=I$, the group $H^{2}\left(A_{\tau}, \mathbb{Q}\right)$ is identified with as the space of alternating rational-valued bilinear maps on $I_{\mathbb{Q}}=B$. For an element $b$ in the space $B_{0}$ of traceless elements of $B,[1]$ defines two elements of $H^{2}(A, \mathbb{Q})$ by

$$
\iota(b):(x, y) \mapsto \operatorname{Tr}(b x \bar{y}), \quad \tilde{\iota}(b):(x, y) \mapsto \operatorname{Tr}(b \bar{x} y)
$$

(with $x$ and $y$ in $I$, or in $B$ ). We quote Theorem 3.10 of [1]:

Theorem 1.4. The following assertions hold:

(i) We have $H^{2}\left(A_{\tau}, \mathbb{Q}\right)=\iota\left(B_{0}\right) \oplus \tilde{\iota}\left(B_{0}\right)$, an orthogonal direct sum with respect to the cup product.

(ii) Given $b$ and $c$ in $B_{0}$, the pairing of $\iota(b)$ and $\iota(c)$ is $\operatorname{disc}(I) \operatorname{Tr}(b \bar{c})$, while that of $\tilde{\iota}(b)$ and $\tilde{\imath}(c)$ to $-\operatorname{disc}(I) \operatorname{Tr}(b \bar{c})$.

(iii) $\quad \iota\left(B_{0}\right) \subseteq H^{1,1}\left(A_{\tau}\right)$, while $\tilde{\iota}\left(B_{0}\right)_{\mathbb{C}}$ is isomorphic to the fiber of $V_{2}$ over $\tau$.

(iv) For $a \in \mathcal{M} \subseteq \operatorname{End}\left(A_{\tau}\right)$ we have $a^{*} \iota(b)=\iota(a b \bar{a})$ and $a^{*} \tilde{\imath}(b)=N(a) \tilde{\iota}(b)$.

(v) Let $\tau, \sigma, I, J$, and $M$ as in Lemma 1.2 be given. Then the equalities $M^{*} \iota(b)=N(M) \iota(b)$ and $M^{*} \tilde{\iota}(b)=\tilde{\iota}(M b \bar{M})$ hold. 
Part (iii) of Theorem 1.4 also includes assertions from Theorem 5.8 and Proposition 5.12 of [1]. Part (v) is a generalization of part (6) of Theorem 3.10 of [1], but the (straightforward) proof extends to this case. We shall later consider these cohomology classes in the de-Rham setting, which will also simplify the proofs of the first three parts of Theorem from Theorem 1.4 we also deduce

Corollary 1.5. Let $\tau, I$, and $A_{\tau}$ be as above.

(i) $\quad$ embeds $\Lambda=\left\{b \in B_{0} \mid \iota(b)(I, I) \subseteq \mathbb{Z}\right\}$ into the Néron-Severi group $N S\left(A_{\tau}\right)$ of $A_{\tau}$.

(ii) $\tilde{\Lambda}=\left\{b \in B_{0} \mid \tilde{\imath}(b)(I, I) \subseteq \mathbb{Z}\right\}$ is an even lattice of signature $(2,1)$.

(iii) Any element of the form $\frac{J_{\sigma}}{\sqrt{\operatorname{disc(I)}}} \in B$, with $\sigma \in \mathcal{H}$, yields via ८ a principal polarization of $A_{\tau}$.

(iv) All the possible polarizations are related by the action of $\mathcal{M}$.

Proof. Part (i) follows from parts (iii) and (v) of Theorem 1.4 together with the Lefschetz Theorem on $(1,1)$ classes. Part (ii) is a consequence of part (ii) of Theorem 1.4. For part (iii) we apply parts (ii) and (iii) of Theorem 1.4 and the positivity condition $-\operatorname{Tr}(v x J \cdot \bar{x})>0$ for $0 \neq x \in B$. Part (iv) follows from part (iv) of that theorem. This proves the corollary.

Part (iii) of Corollary 1.5 justifies, a fortiori, our reference to $A_{\tau}$ as an Abelian surface (rather than just a complex torus). On the other hand, part (iv) shows that the polarization cannot be characterized further than coming from $\iota(\Lambda)$.

Consider now the universal family $\pi: \mathcal{A} \rightarrow \mathcal{H}$, as well as the ones over $X(\Gamma)$ for $\Gamma$ without fixed points. Theorem 1.4 then yields

Corollary 1.6. The variation of Hodge structure $R^{2} \pi_{*} \mathbb{C}$ is the orthogonal direct sum of two variations of Hodge structures. One is the constant variation of Hodge structure $\left(B_{0}\right)_{\mathbb{C}}[1]$. The other one is isomorphic to $V_{2}$, but with the polarization multiplied by disc $(I)$. $\iota$ embeds the lattice $\Lambda$ also into $N S(\mathcal{A})$.

In the case where $B=M_{2}(\mathbb{Q})$ and $\mathcal{M}=I=M_{2}(\mathbb{Z})$, Corollary 1.6 is already established in Section 3 of [10]. Indeed, we have $A_{\tau}=E_{\tau} \times E_{\tau}$ with $E_{\tau}=\mathbb{C} /(\mathbb{Z} \tau \oplus \mathbb{Z})$, and $\mathcal{A}$ is $\mathcal{E} \times \mathcal{H} \mathcal{E}$ for the universal elliptic curve $\pi^{\mathcal{E}}: \mathcal{E} \rightarrow \mathcal{H}$. The group $H^{2}\left(E_{\tau} \times E_{\tau}\right)$ decomposes as

$$
\left(H^{2}\left(E_{\tau}\right) \otimes H^{0}\left(E_{\tau}\right)\right) \oplus\left(H^{0}\left(E_{\tau}\right) \otimes H^{2}\left(E_{\tau}\right)\right) \oplus \bigwedge^{2} H^{1}\left(E_{\tau}\right) \oplus \operatorname{Sym}^{2} H^{1}\left(E_{\tau}\right)
$$

(Künneth and the action of $S_{2}$ on $H^{1}\left(E_{\tau}\right)^{\otimes 2}$ ), where the lattice $\Lambda$ from part $(i)$ of Corollary 1.5 is the direct sum of the first three terms. The lattice $\tilde{\Lambda}$ from part (ii) of that Corollary is the last term. Since $V_{1}=R^{1} \pi_{*}^{\mathcal{E}} \mathbb{C}$, it follows that

$$
R^{2} \pi_{*} \mathbb{C}=\left(R^{2} \pi_{*}^{\mathcal{E}} \mathbb{C} \otimes R^{0} \pi_{*}^{\mathcal{E}} \mathbb{C}\right) \oplus\left(R^{0} \pi_{*}^{\mathcal{E}} \mathbb{C} \otimes R^{2} \pi_{*}^{\mathcal{E}} \mathbb{C}\right) \oplus \bigwedge^{2} V_{1} \oplus V_{2},
$$

with the first three variations of Hodge structures being constant of type $(1,1)$. The cycles from Corollaries 1.5 and 1.6 which are associated with the decompositions are the axes $\{0\} \times E_{\tau}$ and $E_{\tau} \times\{0\}$ (or their unions $\bigcup_{\tau \in \mathcal{H}}\{0\} \times\left. E_{\tau}\right|_{\tau}$ and $\bigcup_{\tau \in \mathcal{H}} E_{\tau} \times\left.\{0\}\right|_{\tau}$ inside the universal family $\mathcal{E} \times \mathcal{H}_{\mathcal{H}} \mathcal{E}$ ) and the normalized diagonal $\widetilde{\Delta}_{E_{\tau}}=\Delta_{E_{\tau}}-\{0\} \times E_{\tau}-E_{\tau} \times\{0\}$ 
(or $\left.\bigcup_{\tau \in \mathcal{H}} \widetilde{\Delta}_{E_{\tau}}\right|_{\tau} \subseteq \mathcal{E} \times \mathcal{H} \mathcal{E}$ ), where $\Delta_{E_{\tau}}$ is the imae of diagonal embedding of $E_{\tau}$ into $A_{\tau}$. More generally, if $\mathcal{M}$ (and $I$ ) is the classical Eichler order of level $N$ in $M_{2}(\mathbb{Q}$ ), so that $\Gamma=\Gamma_{0}(N)$, then the Abelian surface $A_{\tau}$ is $E_{\tau} \times E_{N \tau}$. Then the first axis is $\{0\} \times E_{N \tau}$, and $\widetilde{\Delta}_{E_{\tau}}$ is replaced by the normalized graph of the classical isogeny $E_{\tau} \rightarrow E_{N \tau}$ descending from multiplication by $N$ on $\mathbb{C}$.

\subsection{De-Rham cohomology classes}

The complex multiplication (CM) cycles inside Abelian surfaces with QM are defined in Section 5 of [1] as certain cohomology classes. We shall later define them as actual cycles, generalizing the classical graphs of $\mathrm{CM}$ endomorphisms from the split case. Evaluating the cohomology classes of our cycles is most easily carried out in the de-Rham setting, since it has the advantage that the calculation is independent of the choice of quaternion algebra $B$ with which we work. This is so, since $H_{d R}^{k}\left(A_{\tau}\right) \cong H^{k}\left(A_{\tau}, \mathbb{R}\right)$ is the space of algebraic $k$-forms on the space $T_{0}\left(A_{\tau}\right)=M_{2}(\mathbb{R})$, which is independent of $B$. The Hodge decomposition of the complexification of this space is also independent of $B$. This approach also has the advantage of extending to some base spaces of higher dimensions (indeed, in a subsequent paper we present these results for some 2-dimensional base spaces). Now, writing matrices in $M_{2}(\mathbb{R})$ as $\left(\begin{array}{ll}a & b \\ c & d\end{array}\right)$ defines $a, b, c$, and $d$ as algebraic 1 -forms on $M_{2}(\mathbb{R})$, and we have

Lemma 1.7. Extending scalars to $\mathbb{R}$, the maps $\iota$ and $\tilde{\iota}$ on $\left(B_{0}\right)_{\mathbb{R}}=M_{2}(\mathbb{R})_{0}$ may be evaluated by the equalities

$$
\begin{aligned}
& \iota\left(\begin{array}{cc}
0 & -1 \\
0 & 0
\end{array}\right)=d \wedge c, \quad \iota\left(\begin{array}{cc}
1 & 0 \\
0 & -1
\end{array}\right)=a \wedge d-b \wedge c, \quad \iota\left(\begin{array}{ll}
0 & 0 \\
1 & 0
\end{array}\right)=b \wedge a, \\
& \tilde{\iota}\left(\begin{array}{cc}
0 & -1 \\
0 & 0
\end{array}\right)=c \wedge a, \quad \tilde{\iota}\left(\begin{array}{cc}
1 & 0 \\
0 & -1
\end{array}\right)=d \wedge a+c \wedge b, \quad \tilde{\iota}\left(\begin{array}{ll}
0 & 0 \\
1 & 0
\end{array}\right)=d \wedge b .
\end{aligned}
$$

The proof is straightforward and simple. For the cup product, we prove

Lemma 1.8. The form $a \wedge b \wedge c \wedge d=b \wedge a \wedge d \wedge c=d \wedge c \wedge b \wedge a$ on $A_{\tau}$ is oriented. If $A_{\tau}$ is defined by the ideal I in $B$ then the integral of this form over $A_{\tau}$ equals disc(I).

Proof. The assertion about orientation is immediate. For the integral, we first observe that for $I=M_{2}(\mathbb{Z})$ it indeed equals 1 . Moreover, $M_{2}(\mathbb{Z})$ is self-dual with respect to the pairing $(x, y) \mapsto \operatorname{Tr}(x \bar{y})$ on $M_{2}(\mathbb{R})$. For the general case, let $T \in M_{4}(\mathbb{R})$ be the matrix representing a basis of $I$ over $\mathbb{Z}$ by $a, b, c$, and $d$. The dual basis for $I^{*}=\{x \in$ $\left.M_{2}(\mathbb{R}) \mid(x, I) \subseteq \mathbb{Z}\right\}$ is represented by $\left(T^{t}\right)^{-1}$, so that the definition of the discriminant yields $\operatorname{disc}(I)^{2}=\operatorname{det} T / \operatorname{det}\left(T^{t}\right)^{-1}=(\operatorname{det} T)^{2}$. As the integral in question equals $|\operatorname{det} T|$, the lemma follows.

Tensoring with $\mathbb{C}$ we obtain the Hodge types, which are determined by the following

Lemma 1.9. If $d z_{1}$ and $d z_{2}$ are the holomorphic 1-forms on the holomorphic tangent space $\mathbb{C}^{2}$ of $A_{\tau}$ defined by writing its elements as $\left(\begin{array}{l}z_{2} \\ z_{2}\end{array}\right)$, then the equalities

$$
\begin{aligned}
& d \wedge c=\frac{d z_{2} \wedge d \bar{z}_{2}}{-2 i y}, \quad b \wedge a=\frac{d z_{1} \wedge d \bar{z}_{1}}{-2 i y}, \quad a \wedge d-b \wedge c=\frac{d z_{2} \wedge d \bar{z}_{1}+d z_{1} \wedge d \bar{z}_{2}}{2 i y}, \\
& d z_{2} \wedge d z_{1}=\tilde{\imath}\left(M_{\tau}\right), \quad d \bar{z}_{2} \wedge d \bar{z}_{1}=\tilde{\imath}\left(M_{\bar{\tau}}\right), \quad \text { and } \quad \frac{d z_{2} \wedge d \bar{z}_{1}-d z_{1} \wedge d \bar{z}_{2}}{2}=\tilde{\imath}\left(y J_{\tau}\right)
\end{aligned}
$$

hold. 
Proof. The identification $\mathbb{C}^{2}=M_{2}(\mathbb{R})\left(\begin{array}{l}\tau \\ 1\end{array}\right)$ gives

$$
d z_{1}=a \tau+b, \quad d z_{2}=c \tau+d, \quad d \bar{z}_{1}=a \bar{\tau}+b, \quad \text { and } \quad d \bar{z}_{2}=c \bar{\tau}+d .
$$

The first three equalities follow from a direct calculation. The other equalities are consequences of Lemma 1.7. This proves the lemma.

As Lemma 1.8 shows that the cup product of $\eta$ and $\omega$ in $H^{2}\left(A_{\tau}, \mathbb{R}\right)$ is $N \operatorname{disc}(I)$ if $\eta \wedge$ $\omega=N a \wedge b \wedge c \wedge d$, Lemmas 1.7, 1.8, and 1.9 prove the first three parts of Theorem 1.4 directly. The proof of part (iii) also makes use of the explicit isomorphism between the representations $S y m^{2} \mathbb{R}^{2}$ and $M_{2}(\mathbb{R})_{0}$ of $S L_{2}(\mathbb{R})$ (the action on the latter is by conjugation) given by

$$
\left(\begin{array}{l}
1 \\
0
\end{array}\right)^{2} \longleftrightarrow\left(\begin{array}{rr}
0 & -1 \\
0 & 0
\end{array}\right), \quad\left(\begin{array}{l}
1 \\
0
\end{array}\right)\left(\begin{array}{l}
0 \\
1
\end{array}\right) \longleftrightarrow \frac{1}{2}\left(\begin{array}{rr}
1 & 0 \\
0 & -1
\end{array}\right), \quad \text { and }\left(\begin{array}{l}
0 \\
1
\end{array}\right)^{2} \longleftrightarrow\left(\begin{array}{ll}
0 & 0 \\
1 & 0
\end{array}\right) .
$$

Similar considerations also prove

Corollary 1.10. The fundamental (cohomology) class of a 1-codimensional cycle $C$ in $A_{\tau}$ is represented by

$$
\begin{aligned}
& \frac{1}{\operatorname{disc}(I)}\left[\left(\int_{C} d \wedge c\right) b \wedge a+\left(\int_{C} b \wedge a\right) d \wedge c-\left(\int_{C} d \wedge b\right) c \wedge a+\right. \\
& \left.-\left(\int_{C} c \wedge a\right) d \wedge b+\left(\int_{C} c \wedge b\right) d \wedge a+\left(\int_{C} d \wedge a\right) c \wedge b\right] .
\end{aligned}
$$

Proof. the cycle $C$ is represented, in terms of Poincaré duality, by the 2-form $\omega$, if and only if the equality

$$
\int_{C} \eta=\int_{A_{\tau}} \omega \wedge \eta=\int_{A_{\tau}} \eta \wedge \omega \text { holds for every } \eta \in H^{2}\left(A_{\tau}, \mathbb{C}\right) .
$$

The corollary now follows from Lemma 1.8.

We first recover the expressions for the fundamental classes of the graphs of the generic maps between elliptic curves. Take $I=\mathcal{M}$ to be the classical Eichler order of level $N$ in $B=M_{2}(\mathbb{Q})$. For any $M \in N \mathbb{Z}$ we define $\varphi_{M}: E_{\tau} \rightarrow E_{N \tau}$ to be the map descending from multiplication by $M$ on $\mathbb{C}$. Similarly, if $L \in \mathbb{Z}$ then $\psi_{L}: E_{N \tau} \rightarrow E_{\tau}$ descends from multiplication by $L$ on $\mathbb{C}$. The graphs of these maps are cycles in $A_{\tau}=E_{\tau} \times E_{N \tau}$, for which we indeed obtain

Proposition 1.11. The fundamental class of the graph of $\varphi_{M}$ in $A_{\tau}$ is

$$
\frac{M^{2}}{N} b \wedge a+\frac{M}{N}(a \wedge d-b \wedge c)+\frac{d \wedge c}{N}=\iota\left(\begin{array}{c}
M / N-1 / N \\
M^{2} / N-M / N
\end{array}\right) .
$$

For the graph of $\psi_{L}$ it is

$$
b \wedge a+L(a \wedge d-b \wedge c)+L^{2} d \wedge c=\iota\left(\begin{array}{cc}
L & -L^{2} \\
1 & -L
\end{array}\right) .
$$

Note that the first class here lies in $H^{2}\left(A_{\tau}, \mathbb{Z}\right)$ since $\frac{c}{N} \in H^{1}\left(A_{\tau}, \mathbb{Z}\right)$. 
Proof. The equalities $d z_{2}=M d z_{1}$ and $d \bar{z}_{2}=M d \bar{z}_{1}$ hold along the graph of $\varphi_{M}$, which is defined by the relation $z_{2}=M z_{1}$. This gives $c=M a$ and $d=M b$ by the proof of Lemma 1.9. The integral of $b \wedge a$ over this cycle coincides with its integral over $E_{\tau}$, which equals 1 . Using the relations $c=M a$ and $d=M b$ on this cycle we can evaluate the other integrals appearing in Corollary 1.10. This gives the desired result since $\operatorname{disc}(I)=N$. For the graph of $\psi_{L}$ we get the equalities $a=L c$ and $b=L d$, while the integral of $d \wedge c$ over $E_{N \tau}$ (and over the cycle) is $N$. A similar argument now completes the proof of the proposition.

In particular, the axes $E_{\tau} \times\{0\}$ and $\{0\} \times E_{N \tau}$ are the graphs of $\varphi_{0}$ and $\psi_{0}$ respectively. Proposition 1.11 assigns to these the classes $\frac{d \wedge c}{N}=\iota\left(\begin{array}{cc}0 & -1 / N \\ 0 & 0\end{array}\right)$ and $b \wedge a=\iota\left(\begin{array}{ll}0 & 0 \\ 1 & 0\end{array}\right)$ respectively. The third "basis element" $\iota\left(\begin{array}{cc}1 & 0 \\ 0 & -1\end{array}\right)=a \wedge d-b \wedge c$ is indeed obtained by subtracting the appropriate multiples of the axes from the graphs of either $\varphi_{N}$ or $\psi_{1}$ (which are dual isogenies). Hence for split $B$ the generic subgroup of $N S\left(A_{\tau}\right)$ is generated by classes of Abelian subvarieties of $A_{\tau}$. This is never the case if $B$ is a division algebra-see Theorem 1.19 below.

Next, we consider the classical CM isogenies. Let an imaginary quadratic field $\mathbb{K}=$ $\mathbb{Q}(\sqrt{-D})$ be given. For any $\tau \in \mathcal{H} \cap \mathbb{K}$, both $E_{\tau}$ and $E_{N \tau}$ have $C M$ from an order $\mathcal{O}$ in $\mathbb{K}$. Multiplying $D$ by an appropriate square, we may assume that $\sqrt{-D} \in \mathcal{O}$. Over such an element $\tau$ of $\mathcal{H}$ one can define the $\mathrm{CM}$ isogenies "multiplication by $N \sqrt{-D}$ " from $E_{\tau}$ to $E_{N \tau}$ and "multiplication by $\sqrt{-D}$ " from $E_{N \tau}$ to $E_{\tau}$. Let us reproduce the results about their fundamental classes in $A_{\tau}=E_{\tau} \times E_{N \tau}$ :

Proposition 1.12. The fundamental class of the graph of the latter isogeny is

$$
b \wedge a+D d \wedge c+\frac{\sqrt{D}}{y}\left[|\tau|^{2} c \wedge a+x(d \wedge a+c \wedge b)+d \wedge b\right]=\iota\left(\begin{array}{cc}
0 & -D \\
1 & 0
\end{array}\right)+\tilde{\iota}\left(\sqrt{D} J_{\tau}\right) .
$$

For the former, we get

$$
\frac{d \wedge c}{N}+N D b \wedge a-\frac{\sqrt{D}}{y}\left[|\tau|^{2} c \wedge a+x(d \wedge a+c \wedge b)+d \wedge b\right]
$$

which equals $\iota\left(\begin{array}{cc}0 & -1 / N \\ N D & 0\end{array}\right)-\tilde{\imath}\left(\sqrt{D} J_{\tau}\right)$.

Proof. Along the graph of the CM isogeny $E_{N \tau} \rightarrow E_{\tau}$ we have $d z_{1}=\sqrt{-D} d z_{2}$ and $d \bar{z}_{1}=-\sqrt{-D} d \bar{z}_{2}$, which translate to $\left(\begin{array}{ll}a & b\end{array}\right)=\left(\begin{array}{ll}c & d\end{array}\right) \sqrt{D} J_{\tau}$ (as row vectors of length 2). The integral of $d \wedge c$ over the cycle is $N$ (like with $\psi_{L}$ from Proposition 1.11). Evaluating the other integrals along the cycle by relating the 2-forms to $d \wedge c$ and substituting the results as well as the value $N$ of $\operatorname{disc}(I)$ into Corollary 1.10 yields the asserted value. The equalities satisfied along the graph of the other CM isogeny are $d z_{2}=N \sqrt{-D} d z_{1}$ and $d \bar{z}_{2}=-N \sqrt{-D} d \bar{z}_{1}$, whence $\left(\begin{array}{ll}c & d\end{array}\right)=\left(\begin{array}{ll}a & b\end{array}\right) N \sqrt{D} J_{\tau}$. The integral of $b \wedge a$ along this graph is 1 (as for $\varphi_{M}$ in Proposition 1.11), and again we obtain the required fundamental class. For the description using $\iota$ and $\tilde{\iota}$ we apply Lemma 1.7. This proves the proposition.

The generic parts in the fundamental classes appearing in Proposition 1.12 are the multiples of the axes $E_{\tau} \times\{0\}$ and $\{0\} \times E_{N \tau}$ coming from the intersection numbers. In view of Theorem 1.17 below, we mention that they are equal $\iota\left(\sqrt{D} J_{\sqrt{-D}}\right)$ and $\iota\left(\sqrt{D} J_{-1 / N \sqrt{-D}}\right)$ respectively. The normalized fundamental classes are the transversal classes $\pm \tilde{\imath}\left(\sqrt{D} J_{\tau}\right)$, 
indeed of Hodge type $(1,1)$ by part (iii) of Theorem 1.4. The different signs are related to the fact that these isogenies are not dual to one another, but to minus one another.

\subsection{CM points and $\mathrm{CM}$ cycles}

Let $B$ now be arbitrary, with $i, \mathcal{M}, I, \tau, A_{\tau}$, and $\pi: \mathcal{A} \rightarrow \mathcal{H}$ as above. For every $\tau \in$ $\mathcal{H}, N S\left(A_{\tau}\right)$ contains the generic part $\iota(\Lambda)$ (hence is of rank at least 3 ), but it cannot be of rank exceeding $\operatorname{dim}_{\mathbb{C}} H^{1,1}\left(A_{\tau}\right)=4$. The CM points, which depend on $B$ and on the isomorphism $i$, are characterized by the following extension of Lemma 7.2 of [1]:

Proposition 1.13. For $\tau \in \mathcal{H}$, the following conditions are equivalent:

(i) There exists $b \in B \backslash \mathbb{Q}$ with $N(b)>0$ such that $b=i(b) \in G L_{2}^{+}(\mathbb{R})$ fixes $\tau$.

(ii) Some (real) multiple of $J_{\tau}$ lies in $B=i(B)$.

(iii) The rank of $N S\left(A_{\tau}\right)$ is 4 (rather than the generic 3).

(iv) There is a non-trivial endomorphism of $A_{\tau}$ commuting with the action of $\mathcal{M}$.

Proof. The equivalence of ( $i$ ) and (ii) follows from the fact that the stabilizer of $\tau$ in $G L_{2}^{+}(\mathbb{R})$ consists of the matrices of the form $d I+c J_{\tau}$ with $c$ and $d$ real. (ii) and (iii) are equivalent since $H^{1,1}\left(A_{\tau}\right) \cap \iota\left(B_{0}\right)^{\perp}$ consists of multiples of $\tilde{\iota}\left(J_{\tau}\right)$, using the Lefschetz Theorem on $(1,1)$ classes. Now, Lemma 1.2 implies that endomorphisms of $A_{\tau}$ which commute with the action of $\mathcal{M}$ are given by right multiplication by a matrix from $R(I)$ whose action preserves $\tau$. As such a non-zero element must have a positive reduced norm, the equivalence of (ii) and (iv) also follows. This proves the proposition.

The proof of Proposition 1.13 shows that if $A_{\tau}$ has $\mathrm{CM}$ then $\operatorname{End}_{\mathcal{M}}\left(A_{\tau}\right)$ is generated by some element of the form $b=c J_{\tau}$ of $B$, whose square $-c^{2}=-N(b)$ is rational and negative. Hence $\operatorname{En} d_{\mathcal{M}}\left(A_{\tau}\right)$ is an order in an imaginary quadratic field, which embeds into $B$. This imaginary quadratic field thus splits $B$.

Fixing an imaginary quadratic field $\mathbb{K}$ which splits $B$, we need to normalize certain embeddings. Fix one embedding of $\mathbb{K}$ into $\mathbb{C}$ (which we write just as inclusion), so that henceforth $\sqrt{-D} \in \mathbb{K}$ with positive $D \in \mathbb{Q}$ will mean the element of $\mathbb{K} \cap \mathcal{H}$ via this inclusion. Any embedding of $\mathbb{K}$ into $B \subseteq M_{2}(\mathbb{R})$ sends $\sqrt{-D} \in \mathbb{K}$ to $\pm \sqrt{D} J_{\tau}$ for some $\tau \in \mathcal{H}$. We call this embedding normalized if the sign is positive. Considering the action of the endomorphism $\sqrt{D} J_{\tau}$ of $A_{\tau}$ appearing in part (iv) of Proposition 1.13 on $H^{1,0}\left(A_{\tau}\right)$, one easily verifies that our normalization is equivalent to the one given in Section 7 of [1]. All the embeddings henceforth will be assumed to be normalized.

Recall that in the split case, all the points from $\mathcal{H} \cap \mathbb{K}$ for a fixed imaginary quadratic field $\mathbb{K}$ are related by the action of $G L_{2}^{+}(\mathbb{Q})$. We now extend this assertion to any quaternion algebra $B$. Let $\tau_{0} \in \mathcal{H}$ be the fixed point of a normalized embedding of some imaginary quadratic field $\mathbb{K}$ (of discriminant $-D$ ) into $B$. Thus $A_{\tau_{0}}$ has $C M$ from an order in $\mathbb{K}$ and $\sqrt{D} J_{\tau_{0}} \in B$ by Proposition 1.13. We now prove

Lemma 1.14. For a point $\tau \in \mathcal{H}$, the following are equivalent:

(i) The Abelian surface $A_{\tau}$ (with $Q M$ from $\mathcal{M}$ ) has $C M$ from an order in $\mathbb{K}$.

(ii) $\tau_{0}=\gamma \tau$ for some $\gamma \in B$ with positive norm. 
Proof. Proposition 1.13 shows that condition (i) is equivalent to $\sqrt{D} J_{\tau}$ being in $B$. Combining Eq. (2), the Skolem-Noether Theorem, and the fact that $J_{\tau}$ determines $\tau$ with the fact that det $\gamma$ must be positive if $\tau_{0}=\gamma \tau$ holds with $\tau$ and $\tau_{0}$ from $\mathcal{H}$ now yields the desired equivalence. This proves the lemma.

Scalar multiplication from $\mathbb{Z}$ can take the element $\gamma$ in condition (ii) of Lemma 1.14 to any order or ideal in $B$ of our choice. Moreover, Lemma 1.14 extends to $\tau_{0} \in \overline{\mathcal{H}}$ by allowing $\gamma$ to have negative reduced norm. This will be useful for defining the most general CM cycles in $A_{\tau}$ below.

We consider the graphs of $\mathrm{CM}$ isogenies from the split $B$ case as the images in $A_{\tau}$ of the lines $\mathbb{C}\left(\begin{array}{c}1 \\ N \sqrt{-D}\end{array}\right)$ and $\mathbb{C}\left(\begin{array}{c}\sqrt{-D} \\ 1\end{array}\right)$ in the universal cover $\mathbb{C}^{2}$ respectively. Multiplying $D$ by an integral square changes the slopes of these lines, yielding many CM cycles in $A_{\tau}$. The graphs of the generic maps $\varphi_{M}$ with $M \in N \mathbb{Z}$ or $\psi_{L}$ for $L \in \mathbb{Z}$ are described similarly, using the lines $\mathbb{C}\left(\begin{array}{l}1 \\ M\end{array}\right)$ and $\mathbb{C}\left(\begin{array}{l}L \\ 1\end{array}\right)$ respectively. With this motivation, let $B$ (and $i$ ) be general, let $\tau_{0} \in \mathcal{H} \cup \overline{\mathcal{H}}$ and $\mathbb{K}$ be as in (the extended version of) Lemma 1.14, and assume that the Abelian surface $A_{\tau}$ with QM has also CM from an order in $\mathbb{K}$. We now prove

Proposition 1.15. The image of the line $\mathbb{C}\left(\begin{array}{c}\tau_{0} \\ 1\end{array}\right) \subseteq \mathbb{C}^{2}$ in $A_{\tau}$ is an Abelian subvariety of $A_{\tau}$. Furthermore, it is isomorphic to an elliptic curve with $C M$ from $\mathbb{K}$.

Proof. It suffices to show that the intersection $I\left(\begin{array}{l}\tau \\ 1\end{array}\right) \cap \mathbb{C}\left(\begin{array}{c}\tau_{0} \\ 1\end{array}\right)$ is a full lattice in that line. Lemma 1.14 produces an element $\gamma \in B$ such that $\gamma \tau=\tau_{0}$, which we assume to lie in $I$ and be primitive there. Hence $\gamma\left(\begin{array}{l}\tau \\ 1\end{array}\right) \in I\left(\begin{array}{l}\tau \\ 1\end{array}\right)$ lies in $\mathbb{C}\left(\begin{array}{c}\tau_{0} \\ 1\end{array}\right)$ by Eq. (1). But $\sqrt{D} J_{\tau} \in B$ by Proposition 1.13, and by renormalizing $D$ we may assume that this element lies in $R(I)$. As $\sqrt{D} J_{\tau}$ multiplies $\left(\begin{array}{l}\tau \\ 1\end{array}\right)$ by $\sqrt{-D} \in \mathbb{C} \backslash \mathbb{R}$ (Eq. (1) again), we find that $\gamma \cdot \sqrt{D} J_{\tau}\left(\begin{array}{l}\tau \\ 1\end{array}\right)$ is another element of $\mathbb{C}\left(\begin{array}{c}\tau_{0} \\ 1\end{array}\right)$, which is linearly independent of the previous one over $\mathbb{R}$. Hence the image of $\mathbb{C}\left(\begin{array}{c}\tau_{0} \\ 1\end{array}\right)$ in $A_{\tau}$ is an Abelian subvariety of $A_{\tau}$, which is an elliptic curve. As $\gamma$ is primitive in $I$, we may find integers $g>0$ and $h$ such that $\gamma\left(\begin{array}{l}\tau \\ 1\end{array}\right)$ and $\gamma \frac{\sqrt{D} J_{\tau}-h}{g}\left(\begin{array}{l}\tau \\ 1\end{array}\right)$ generate the lattice $I\left(\begin{array}{l}\tau \\ 1\end{array}\right) \cap \mathbb{C}\left(\begin{array}{c}\tau_{0} \\ 1\end{array}\right)$. Our elliptic curve is thus isomorphic to $E_{\tilde{\tau}}$ for $\tilde{\tau}=\frac{\sqrt{-D}-h}{g} \in \mathcal{H}$, indeed having $C M$ by $\sqrt{-D} \in \mathbb{K}$. This proves the proposition.

Proposition 1.15 allows us to make the following

Definition 1.16. Given $\tau$ and $\tau_{0}$ as above, we define the CM cycle corresponding to $\tau_{0}$ in $A_{\tau}$ to be the cycle described in Proposition 1.15.

Definition 1.16 includes the case of graphs of CM isogenies in the split case from above, as the cases $\tau_{0}=\sqrt{-D} \in \mathcal{H}$ and $\tau_{0}=\frac{1}{N \sqrt{-D}} \in \overline{\mathcal{H}}$ respectively. The graphs of the generic maps may be seen as an extension of Definition 1.16 to some rational (or infinite) $\tau_{0}-$ more on this below.

Let $\tau_{0}=x_{0}+i y_{0} \in \mathcal{H} \cup \overline{\mathcal{H}}$ be as above. We prove

Theorem 1.17. The fundamental class of the CM cycle corresponding to $\tau_{0}$ equals $\operatorname{sgn}\left(y_{0}\right)\left[\iota\left(\beta \sqrt{D} J_{x_{0}+i\left|y_{0}\right|}\right)+\tilde{\iota}\left(\beta \sqrt{D} J_{\tau}\right)\right]$ for some positive $\beta \in \mathbb{Q}$.

Proof. The proof of Proposition 1.15 shows that as a subset of $A_{\tau}$, the CM cycle in question is $\mathbb{C}\left(\begin{array}{c}\tau_{0} \\ 1\end{array}\right) / j(\gamma, \tau)(\mathbb{Z} \tilde{\tau} \oplus \mathbb{Z})\left(\begin{array}{c}\tau_{0} \\ 1\end{array}\right)$ (recall the coefficient $j(\gamma, \tau)$ in Eq. (1)). Along this 
cycle the relations $d z_{1}=\tau_{0} d z_{2}$ and $d \bar{z}_{1}=\bar{\tau}_{0} d \bar{z}_{2}$ hold, and in $A_{\tau}$ these relations are equivalent to $\left(\begin{array}{ll}a & b\end{array}\right)=\left(\begin{array}{ll}c & d\end{array}\right)\left(x_{0} I+y_{0} J_{\tau}\right)$. As in Propositions 1.11 and 1.12, evaluating the integral of $d \wedge c$ over our cycle suffices for determining the required fundamental class. Using the first formula in Lemma 1.9, we evaluate the integral of $d z_{2} \wedge d \bar{z}_{2}$ instead. Now, the isomorphism of our cycle with $E_{\tilde{\tau}}$ is given by the second coordinate divided by $j(\gamma, \tau)$. Hence we may substitute $d z_{2}=j(\gamma, \tau) d z$ and $d \bar{z}_{2}=\overline{j(\gamma, \tau)} d \bar{z}$, where $z$ is the coordinate of $E_{\tilde{\tau}}$. A straightforward evaluation now gives

$$
\int_{\left(\mathbb{C}\left(\begin{array}{c}
\tau_{0} \\
1
\end{array}\right) \rightarrow A_{\tau}\right)} d z_{2} \wedge d \bar{z}_{2}=|j(\gamma, \tau)|^{2} \int_{E_{\tilde{\tau}}} d z \wedge d \bar{z}=-2 i|j(\gamma, \tau)|^{2} \Im \tilde{\tau}=-2 i y \frac{\operatorname{det} \gamma \cdot \Im \tilde{\tau}}{y_{0}}
$$

where in the last step we have used the equality $y_{0}=\frac{\operatorname{det} \gamma \cdot y}{|j(\gamma, \tau)|^{2}}$ (recall that $\tau_{0}=\gamma \tau$, and $\operatorname{det} \gamma$ is not necessarily 1 ). Dividing by $-2 i y$ to get the integral of $d \wedge c$ and evaluating the other integrals appearing in Corollary 1.10 as in the proofs of Propositions 1.11 and 1.12 gives the expression

$$
\frac{\operatorname{det} \gamma \cdot \Im \tilde{\tau}}{\operatorname{disc}(I)}\left[\frac{b \wedge a}{y_{0}}+\frac{\left|\tau_{0}\right|^{2}}{y_{0}} d \wedge c+\frac{|\tau|^{2}}{y} c \wedge a+\frac{d \wedge b}{y}+\left(\frac{x}{y}-\frac{x_{0}}{y_{0}}\right) d \wedge a+\left(\frac{x}{y}+\frac{x_{0}}{y_{0}}\right) c \wedge b\right]
$$

for the fundamental class in question. Lemma 1.7 reduces the latter expression to $\frac{\operatorname{det} \gamma \cdot \widetilde{\tau} \tilde{\tau}}{\operatorname{disc}(I)}\left(\iota\left(J_{\tau_{0}}\right)+\tilde{\iota}\left(J_{\tau}\right)\right)$. Now, the coefficient $\frac{\operatorname{det} \gamma \cdot \Im \tilde{\tau}}{\operatorname{disc}(I)}$ equals $\tilde{\beta} \sqrt{D}$ for some $\tilde{\beta} \in \mathbb{Q}$ having the same sign as det $\gamma$ as well as $y_{0}$. Putting $\beta=|\tilde{\beta}|$ and using the relation $J_{\tau_{0}}=-J_{\overline{\tau_{0}}}$ if $\tau_{0} \in \overline{\mathcal{H}}$ completes the proof of the theorem.

The proofs of Proposition 1.15 and Theorem 1.17 involve the parameter $\tilde{\tau}$, which depends on the choice of the primitive element $\gamma \in I$ taking $\tau$ to $\tau_{0}$. However, choosing another such element $\delta$ and taking the appropriate $g$ and $h$ can be seen to yield the index $\alpha \tilde{\tau}$ for some $\alpha \in S L_{2}(\mathbb{Z})$. Moreover, $\gamma^{-1} \delta$ has reduced norm $|j(\alpha, \tilde{\tau})|^{2}$ in this case. As $E_{\alpha \tilde{\tau}} \cong E_{\tilde{\tau}}$ and $\operatorname{det} \delta \cdot \Im \alpha \tilde{\tau}=\operatorname{det} \gamma \cdot \Im \tilde{\tau}$, both the description of the CM cycle and the coefficient we wrote as $\tilde{\beta} \sqrt{D}$ are intrinsic. The actual value of $\beta$ is probably the minimal positive rational number such that both terms give elements of $H^{2}\left(A_{\tau}, \mathbb{Z}\right)$, but this point requires further investigation.

We remark that the generic cycles in the split $B$ case can be obtained as the case $\tau_{0} \in$ $\mathbb{P}^{1}(\mathbb{R})$ of Proposition 1.15 and Theorem 1.17. Indeed, by keeping $|j(\gamma, \tau)|^{2}$ instead of the (here undefined) expression $\frac{\operatorname{det} \gamma}{y_{0}}$ in Eq. (3), the same argument works if det $\gamma=0$ and $\tau_{0} \in \mathbb{R}$. Similar manipulations extend the assertion to $\tau_{0}=\infty$ as well. Note that the sign of the normalized cycle from Theorem 1.17 distinguishes between lines having slopes in $\mathcal{H}$ from those with slopes in $\overline{\mathcal{H}}$ (whence the signs in Proposition 1.12). The vanishing of the $\tilde{\iota}$ part for $\tau_{0} \in \mathbb{P}^{1}(\mathbb{R})$ in Proposition 1.11 corresponds to the fact that $\mathbb{P}^{1}(\mathbb{R})$ lies between $\mathcal{H}$ and $\overline{\mathcal{H}}$.

We remark that this method of calculating fundamental classes of CM cycles is equivalent to the more direct approach of finding intersection numbers of the cycles themselves (see, e.g., the proof of Corollary 1.10). However, it has the advantage of avoiding the need to analyze the structure of the specific quaternion algebra $B$. We also note that our settheoretic presentation depends on the embedding $i$. However, by the Skolem-Noether Theorem, this makes no essential difference algebraically.

An Abelian surface $A_{\tau}$ with QM having $C M$ from an order in a field $\mathbb{K}$ contains infinitely many different $\mathrm{CM}$ cycles. For these we prove 
Proposition 1.18. For $\tau$ as above we have

(i) The action of $\mathcal{M} \subseteq \operatorname{End}\left(A_{\tau}\right)$ relates all the $C M$ cycles in $A_{\tau}$ to one another.

(ii) Given $M \in R(I)_{+}^{\times}$, the isomorphism $A_{M \tau} \rightarrow A_{\tau}$ from Lemma 1.2 takes the $C M$ cycle corresponding to $\tau_{0}$ in $A_{M \tau}$ to the one in $A_{\tau}$.

(iii) If $A_{\tau}$ and its $Q M$ and $C M$ endomorphisms are defined over a number field $\mathbb{F}$ then all the $C M$ cycles are also defined over $\mathbb{F}$.

(iv) $A_{\tau}$ is isogenous, over $\mathbb{F}$, to the self-product of an elliptic curve with $C M$ from an order in $\mathbb{K}$.

Proof. Part (i) follows from Lemma 1.14. For part (ii), Lemma 1.2 shows that the isomorphism in question multiplies the holomorphic tangent space $\mathbb{C}^{2}$ by a scalar. Thus, this map preserves lines in $\mathbb{C}^{2}$, hence also CM cycles. To prove part (iii) note that Eq. (1) characterizes $\mathbb{C}\left(\begin{array}{c}\tau_{0} \\ 1\end{array}\right)$ as those vectors in $\mathbb{C}^{2}$ on which the QM endomorphism $\sqrt{D} J_{\tau_{0}} \in \mathcal{M} \subseteq B$ acts like the $\mathrm{CM}$ endomorphism $\sqrt{-D} \in \mathcal{O} \subseteq \mathbb{K} \subseteq \mathbb{C}$ (with positive imaginary part). By connectedness, the CM cycle corresponding to $\tau_{0}$ is thus the connected component of the kernel of the endomorphism $\sqrt{D} J_{\tau_{0}}-\sqrt{-D} \in \mathcal{M} \otimes \mathcal{O}=$ $\operatorname{End}\left(A_{\tau}\right)$. The kernel itself is clearly defined over $\mathbb{F}$. For the connected component, observe that any automorphism of $\mathbb{C} / \mathbb{F}$ gives an isomorphism of Abelian varieties from $A_{\tau}$ to itself, preserves the kernel in question, takes connected components to connected components, and maps 0 to 0 . Hence any such automorphism preserves the CM cycle in question (this argument was shown to me by Philipp Habegger), which completes the proof of part (iii). For part (iv) we recall that embedding two different CM cycles into $A_{\tau}$ defines an isogeny between the product of these CM cycles and $A_{\tau}$, and that any two elliptic curves with $C M$ from $\mathbb{K}$ are isogenous. The desired assertion now follows from part (iii) and Proposition 1.15. This proves the proposition.

Part (i) of Proposition 1.18 shows that like with polarizations, there is no "canonical" choice of a CM cycle in $A_{\tau}$. Part (ii) implies that the CM cycles are well-defined also on fibers over $X(\Gamma)$ for $\Gamma \subseteq R(I)_{+}^{\times}$. They may therefore be considered as cycles of codimension 2 in the (algebraic) universal family $\mathcal{A}$ over $X(\Gamma)$. Parts (iii) and (iv) essentially appear in Section 7 of [1], but our proof is simpler and more elementary. The first three assertions of Proposition 1.18 extend to the generic cycles in the split $B$ case, when part $(i)$ is interpreted appropriately.

We now have two kinds of (1-dimensional) Abelian subvarieties of an Abelian surface $A$ with QM: The split $B$ case gives rise to generic cycles (graphs of generic correspondences), while the CM case (with general $B$ ) introduces $\mathrm{CM}$ cycles. On the other hand, we can prove

Theorem 1.19. For $\tau \in \mathcal{H}$ we have

(i) If the Abelian surface $A_{\tau}$ with $Q M$ has also $C M$ then the lines with finite non-real slopes in $\mathbb{C}^{2}$ which give Abelian subvarieties of $A_{\tau}$ are exactly the lines descending to $C M$ cycles as in Proposition 1.15.

(ii) If $A_{\tau}$ has no $C M$ then no lines of non-real slopes give Abelian subvarieties of $A_{\tau}$.

(iii) If $B=M_{2}(\mathbb{Q})$ then a line of slope from $\mathbb{P}^{1}(\mathbb{R})$ gives an Abelian subvariety of $A_{\tau}$ if and only if the slope is in $\mathbb{P}^{1}(\mathbb{Q})$.

(iv) If $B$ is not split then no lines of real or infinite slope give Abelian subvarieties of $A_{\tau}$. 
Proof. We need to find all slopes $\tau_{0}$ such that $I\left(\begin{array}{l}\tau \\ 1\end{array}\right)$ intersects $\mathbb{C}\left(\begin{array}{c}\tau_{0} \\ 1\end{array}\right)$ in a full lattice in this line. Equivalently, by Eq. (1) and its extensions we need two linearly independent elements of $B$ which take $\tau$ to $\tau_{0}$. Now, for part (i) we must have $\tau_{0}=\gamma \tau$ for $\gamma \in B^{\times}$, and the assertion follows as in Proposition 1.13 and Lemma 1.14. Now, for two elements $\gamma$ and $\delta$ sending $\tau$ to $\tau_{0}, \gamma^{-1} \delta$ stabilizes $\tau$. Hence part (ii) follows from Proposition 1.13 as well. In addition, the equality $\tau_{0}=\gamma \tau$ with $\tau \in \mathcal{H}, \gamma \neq 0$, and $\tau_{0} \in \mathbb{P}^{1}(\mathbb{R})$ implies $N(\gamma)=0$. Part (iv) is thus also established. For part (iii), recall that an element $0 \neq \gamma \in M_{2}(\mathbb{Q})$ satisfies $\operatorname{det} \gamma=0$ if and only if its rows are rational multiples of one another. Hence only slopes from $\mathbb{P}^{1}\left(\mathbb{Q}\right.$ ) may be obtained. Finally, for $\tau_{0}=\frac{L}{M} \in \mathbb{P}^{1}\left(\mathbb{Q}\right.$ ) (with $\infty=\frac{1}{0}$ ), the elements $\left(\begin{array}{ll}0 & L \\ 0 & M\end{array}\right)$ and $\left(\begin{array}{ll}L & 0 \\ M & 0\end{array}\right)$ of $M_{2}(\mathbb{Q})$ send $\left(\begin{array}{l}\tau \\ 1\end{array}\right)$ to $\left(\begin{array}{l}L \\ M\end{array}\right)$ and $\tau\left(\begin{array}{l}L \\ M\end{array}\right)$, respectively. Thus, lines with such slopes do give Abelian subvarieties, which proves (iii). This completes the proof of the theorem.

Theorem 1.19 shows that the only Abelian subvarieties of an Abelian surface $A$ with QM are the CM cycles (if they exist), plus the generic cycles if $B$ splits. This result is related to rational points on Brauer-Severi varieties (the algebra is $\operatorname{End}(A)_{\mathbb{Q}}$ considered over its center, the latter being $\mathbb{K}$ in the case of CM from $\mathbb{K}$ and $\mathbb{Q}$ if $A$ has no CM). Recall that any Abelian subvariety $C$ of an Abelian variety $A$ admits a "complementary" subvariety $\widetilde{C}$ of $A$ such that $A$ is isogenous to $C \times \widetilde{C}$ (see, for example, the proof of Proposition 10.1 of [20]). Expressing the latter result in terms of (connected components of) kernels of endomorphisms of $A$ shows, together with the proof of part (iii) of Proposition 1.18 here (extended also to the generic cycles), that our Theorem 1.19 agrees with the expected result. However, it also provides an explicit, set-theoretic description of the Abelian subvarieties in this case of Abelian surfaces with QM.

Let $\mathcal{A}^{m}$ be the fibered product of $\mathcal{A}$ with itself $m$ times over $\mathcal{H}$, with the projection $\pi_{m}: \mathcal{A}^{m} \rightarrow \mathcal{H}$. Given a CM cycle $C \subseteq A_{\tau}$, we define its $m$ th power to be

$$
C^{m}=\left\{\left(z_{1}, \ldots, z_{m}\right) \in A_{\tau}^{m} \mid z_{j} \in C, 1 \leq j \leq m\right\} \subseteq A_{\tau}^{m}
$$

This $m$-cycle in $A_{\tau}^{m}$ is also called a CM cycle. We consider $C^{m}$ as a vertical cycle in $\mathcal{A}^{m}$, which is algebraic in $A_{\tau}^{m}$ as well as in $\mathcal{A}^{m}$ over $X(\Gamma)$. Such a cycle has a fundamental cohomology class in $H^{2 m}\left(A_{\tau}^{m}\right)$, or more precisely in the $S_{m}$-invariant part $\operatorname{Sym}^{m} H^{2}\left(A_{\tau}\right)$ of the Künneth component $H^{2}\left(A_{\tau}\right)^{\otimes m}$ of the latter cohomology group. By letting $\tau$ vary, the normalized fundamental classes of these cycles give elements of the fiber of the symmetric part $\operatorname{Sym}^{m} V_{2}$ of the subvariation of Hodge structure $V_{2}^{\otimes m} \subseteq\left(R^{2} \pi_{*} \mathbb{C}\right)^{\otimes m}$ of $R^{2 m} \pi_{m, *} \mathbb{C}$ over $\mathcal{H}$ or over $X(\Gamma)$. Now, $\operatorname{Sym}^{m} V_{2}$ admits a smaller rational subvariation of Hodge structure which is isomorphic to $V_{2 m}$ (it is defined as the kernel of a certain Laplacian map to $S y m^{m-2} V_{2}$ ). The objects referred to as CM cycles in Section 5 of [1] are the images of the normalized fundamental classes of our $m$-dimensional $C M$ cycles under the projection onto this subvariation of Hodge structure (denoted $P$ in that reference). Theorem 4.6 below proves the modularity of our cycles in the larger subvariation of Hodge structure $\operatorname{Sym}^{m} V_{2}$. We note that [13] and [14] have related modularity results, but with the local system $V_{2 m}$ (using other means like the Shintani and Kudla-Millson lifts). For the split $B$ case, the $m$-codimensional cycles arising from $\tau_{0}=\sqrt{-D}$ resemble the cycles defined in page 123 of [31] (for $m=k-1$ ). The action of the larger group $S_{2 m}$ (rather than our $S_{m}$ ) appearing in [31] is the incarnation of the projector $P$ in this case. 


\section{Differential operators on theta functions}

In this Section we prove a relation between the actions of two Laplacians on Siegel theta functions with polynomials of a specific type. This relation is a crucial step in showing that the theta lifts considered in the next Section are eigenfunctions of the Laplacian on the Grassmannian. This property is central in the following applications.

\subsection{The basic summand in a theta function}

Let $V$ be a real vector space with a bilinear form $(\cdot, \cdot): V \times V \rightarrow \mathbb{R}$, which is nondegenerate with signature $\left(b_{+}, b_{-}\right)$. We shorthand $(\lambda, \lambda)$ to $\lambda^{2}$. Hence the associated quadratic form, denoted $q$ in [6], is $\lambda \mapsto \frac{\lambda^{2}}{2}$. Consider the Grassmannian

$$
G(V)=\left\{V=v_{+} \oplus v_{-} \mid v_{+} \gg 0, v_{-} \ll 0, v_{+} \perp v_{-}\right\}
$$

(i.e., $v_{+}$is positive definite and $v_{-}$is negative definite) of $V$, in elements of which we have $\operatorname{dim} v_{ \pm}=b_{ \pm}$. Given $\lambda \in V$, its projection onto $v_{ \pm}$in a given element of $G(V)$ is denoted $\lambda_{v_{ \pm}}$. The Laplacian of $v_{ \pm}$is $\Delta_{v_{ \pm}}= \pm \sum_{h=1}^{b_{ \pm}} \frac{\partial^{2}}{\partial \lambda_{h}^{2}}$ using an orthonormal basis for $v_{ \pm}$. The Laplacian $\Delta_{V}$ of $V$ is $\Delta_{v_{+}}+\Delta_{v_{-}}$(this is independent of the choice of the decomposition), and the Laplacian corresponding to $v \in G(V)$ is $\Delta_{v}=\Delta_{v_{+}}-\Delta_{v_{-}}$(this operator is based on the majorant corresponding to $v$ ). For $\tau \in \mathcal{H}, v \in G(V)$, a polynomial $p$ on $V$, and $\lambda \in V$, define the function

$$
F(\tau, v, p, \lambda)=e^{-\Delta_{v} / 8 \pi y}(p)(\lambda) \mathbf{e}\left(\tau \frac{\lambda_{v_{+}}^{2}}{2}+\bar{\tau} \frac{\lambda_{v_{-}}^{2}}{2}\right)
$$

where $\mathbf{e}(z)=e^{2 \pi i z}$ for any complex number $z$. The polynomial $p$ involved with these functions will usually be homogenous of degree $\left(m_{+}, m_{-}\right)$with respect to $v$. This means that for $\lambda \in V$, which decomposes as $\lambda_{v_{+}}+\lambda_{v_{-}}$with respect to $v$, and two numbers $\alpha_{ \pm} \in \mathbb{R}$, we have $p\left(\alpha_{+} \lambda_{v_{+}}+\alpha_{-} \lambda_{\nu_{-}}\right)=\alpha_{+}^{m_{+}} \alpha_{-}^{m_{-}} p(\lambda)$.

An even lattice in $V$ is a discrete subgroup $L \subseteq V$ of maximal rank $b_{+}+b_{-}$such that $\lambda^{2} \in 2 \mathbb{Z}$ for any $\lambda \in L$. The dual lattice $L^{*}=\operatorname{Hom}(L, \mathbb{Z})$ is a subgroup of $V$ which contains $L$ with finite index $\Delta_{L}$. The Siegel theta function of $L$, with respect to the element $v \in G(V)$ and a polynomial $p$ which is homogenous of degree $\left(m_{+}, m_{-}\right)$with respect to $v$, is the $\mathbb{C}\left[L^{*} / L\right]$-valued function

$$
\Theta_{L}(\tau, v, p)=\sum_{\gamma \in L^{*} / L} \theta_{\gamma}(\tau, v, p) e_{\gamma}, \quad \theta_{\gamma}(\tau, v, p)=\sum_{\lambda \in L+\gamma} F(\tau, v, p, \lambda) .
$$

Here $e_{\gamma}$ is the canonical basis element of $\mathbb{C}\left[L^{*} / L\right]$ which corresponds to the element $\gamma \in L^{*} / L$.

The group $S L_{2}(\mathbb{R})$ admits a double cover, known as the The metaplectic group $M p_{2}(\mathbb{R})$. Its elements are pairs $(M, \varphi)$ with $M \in S L_{2}(\mathbb{R})$ and $\varphi: \mathcal{H} \rightarrow \mathbb{C}$ holomorphic such that $\varphi^{2}(\tau)=j(M, \tau)$ for every $\tau \in \mathcal{H}$. The product rule in $M p_{2}(\mathbb{R})$ takes $(M, \varphi)$ and $(N, \psi)$ to $(M N,(\varphi \circ N) \cdot \psi)$. Let $M p_{2}(\mathbb{Z})$ denote the inverse image of $S L_{2}(\mathbb{Z})$ in $M p_{2}(\mathbb{R})$. It is generated by the two elements $T=\left(\left(\begin{array}{cc}1 & 1 \\ & 1\end{array}\right), 1\right)$ and $S=\left(\left({ }^{-1}{ }^{-1}\right), \sqrt{\tau} \in \mathcal{H}\right)$, satisfying the relation $S^{2}=(S T)^{3}$. This common element $Z=(-I, i)$ has order 4 and generates the center of $M p_{2}(\mathbb{Z})$ (as well as that of $M p_{2}(\mathbb{R})$ ). The kernel of the projection to $S L_{2}(\mathbb{R})$ is generated by $Z^{2}=(I,-1)$. Let $\rho_{V}$ be the Weil representation of $M p_{2}(\mathbb{R})$ on the space $\mathcal{S}(V)$ of Schwartz functions on $V$. Explicitly, let $\left(M=\left(\begin{array}{ll}a & b \\ c & d\end{array}\right), \varphi\right)$ and a Schwartz function 
$\Phi$ on $V$ be given. If $c=0$, so that $\varphi(\tau)$ is the constant $\delta \sqrt{|d|}$ for some $\delta \in\{ \pm 1, \pm i\}$, then $\rho_{V}(M, \varphi) \Phi$ is the function

$$
\lambda \mapsto \delta^{b_{-}-b_{+}}|a|^{\left(b_{+}+b_{-}\right) / 2} \Phi(a \lambda) \mathbf{e}\left(\frac{a b \lambda^{2}}{2}\right) .
$$

Otherwise, it gives the function sending $\lambda \in V$ to

$$
\left(\operatorname{sgn}(\Re \varphi(\tau)) \zeta_{8}^{\operatorname{sgn}(c)}\right)^{b_{-}-b_{+}}|c|^{-\left(b_{+}+b_{-}\right) / 2} \int_{V} \Phi(\mu) \mathbf{e}\left[\frac{d \mu^{2}}{2 c}-\frac{(\lambda, \mu)}{c}+\frac{a \lambda^{2}}{2 c}\right] d \mu .
$$

Here $\zeta_{8}=\mathbf{e}\left(\frac{1}{8}\right)$ is the basic 8th root of unity. Furthermore, we denote $\rho_{L}$ the Weil representation of $M p_{2}(\mathbb{Z})$ on $\mathbb{C}\left[L^{*} / L\right]$, which is defined on the generators by the wellknown formulae

$$
\begin{aligned}
& \rho_{L}(T)\left(e_{\gamma}\right)=\mathbf{e}\left(\gamma^{2} / 2\right) e_{\gamma}, \\
& \rho_{L}(S)\left(e_{\gamma}\right)=\frac{\zeta_{8}^{b_{-}-b_{+}}}{\sqrt{\Delta_{L}}} \sum_{\delta \in L^{*} / L} \mathbf{e}(-(\gamma, \delta)) e_{\delta}
\end{aligned}
$$

(see, for example, Section 4 of [2] or Section 2 of [3]). The number $\zeta_{8}^{b_{+}-b_{-}}$is the Weil index of $V$ arising from its quadratic structure and the character $t \mapsto \mathbf{e}(t)$ of $\mathbb{R}$, as well as of the discriminant form $L^{*} / L$. This representation factors through a double cover of $S L_{2}(\mathbb{Z} / N \mathbb{Z})$ for some integer $N$ called the level of $L$, or through $S L_{2}(\mathbb{Z} / N \mathbb{Z})$ itself if the signature of $L$ is even. The action of a general element of $M p_{2}(\mathbb{Z})$ via $\rho_{L}$ is given in in [22] for even signature and [26] or [28] for the general case.

Let $K$ be the stabilizer of $i \in \mathcal{H}$ in $M p_{2}(\mathbb{R})$. It is a maximal compact subgroup of $M p_{2}(\mathbb{R})$. It consists of the elements $k_{\theta}=\left(\left(\begin{array}{cc}\cos \theta & -\sin \theta \\ \sin \theta & \cos \theta\end{array}\right), \varphi\right)$, where $\varphi(i)=e^{i \theta / 2}$, for $\theta \in \mathbb{R} / 4 \pi \mathbb{Z}$. Some properties of the functions $F$ and $\Theta_{L}$, which will turn out useful in this paper, are summarized in the following

Theorem 2.1. Assume that the polynomial $p$ is homogenous of degree $\left(m_{+}, m_{-}\right)$with respect to $v$.

(i) For any $M \in M p_{2}(\mathbb{R})$ we have the equality

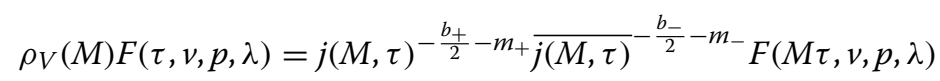

of functions of $\lambda \in V$.

(ii) The action of $\rho_{V}\left(k_{\theta}\right)$ multiplies $F(i, v, p, \lambda)$ by $\left(e^{i \theta}\right)^{\frac{b_{-}}{2}+m_{-}-\frac{b_{+}}{2}-m_{+}}$.

(iii) If $M \in M p_{2}(\mathbb{Z})$ then the equality

$$
\rho_{L}(M) \Theta_{L}(\tau, v, p)=j(M, \tau)^{-\frac{b_{+}}{2}-m_{+} \overline{j(M, \tau)}-\frac{b_{-}}{2}-m_{-}} \Theta_{L}(M \tau, v, p)
$$

holds in $\mathbb{C}\left[L^{*} / L\right]$.

Proof. Part (i) is essentially Lemma 1.2 of [25] (in fact, only the case $b_{+}=2$ and certain harmonic polynomials of homogeneity degree $\left(m_{+}, 0\right)$ are considered there, but using the results of Section 3 of [2] the proof extends to the general case). Part (ii) is the special case in which we take $M=k_{\theta} \in K$ and $\tau=i$ in part ( $i$ ). Part (iii) is the case $\alpha=\beta=0$ in Theorem 4.1 of [2]. This proves the theorem. 
Given real $r$ and $s$, let $\widetilde{\Delta}_{r, s}$ denote the operator

$$
y^{2}\left(\partial_{x}^{2}+\partial_{y}^{2}\right)-i y(r-s) \partial_{x}+y(r+s) \partial_{y}=4 y^{2} \partial_{\tau} \partial_{\bar{\tau}}-2 i r y \partial_{\bar{\tau}}+2 i s \partial_{\tau}
$$

on functions on $\mathcal{H}$. The operator $\Delta_{r, s}=\widetilde{\Delta}_{r, s}+(r-1) s$ is the weight $(r, s)$ Laplacian on $\mathcal{H}$, and the equality $\Delta_{r, s} y^{t}=y^{t} \Delta_{r+t, s+t}$ holds for any $t$. The weight $r$ Laplacian is just $\Delta_{r}=\Delta_{r, 0}$. On the other hand, let $\Delta_{S_{b_{+}, b_{-}}^{+}}$denote the Laplacian of $O(V)$, i.e., the Casimir element of the universal enveloping algebra of $\mathfrak{s o}_{b_{+}, b_{-}}$. We shall need the following

Proposition 2.2. Let $\tau \in \mathcal{H}, \lambda \in V$, and $v \in G(V)$ be as above, and let $p$ be as in Theorem 2.1. Denote $\frac{b_{+}}{2}+m_{+}-\frac{b_{-}}{2}-m_{-}$by $k$. Then we have the equality

$$
\begin{aligned}
& \Delta_{S O_{b_{+}, b_{-}}^{+}, \lambda} y^{\frac{b_{-}}{2}+m_{-}} F(\tau, v, p, \lambda)=4 \Delta_{k, \tau} y^{\frac{b_{-}}{2}+m_{-}} F(\tau, v, p, \lambda)+ \\
& +\left[\left(m_{+}-m_{-}\right)\left(m_{+}-m_{-}+b_{+}-b_{-}-2\right)-\left(b_{+}-2\right) b_{-}\right] y^{\frac{b_{-}}{2}+m_{-}} F(\tau, v, p, \lambda) .
\end{aligned}
$$

Proof. Given $\tau \in \mathcal{H}$ define $g_{\tau}=\left(\frac{1}{\sqrt{y}}\left(\begin{array}{cc}y & x \\ 1\end{array}\right), \varphi \equiv+\frac{1}{\sqrt[4]{y}}\right) \in M p_{2}(\mathbb{R})$. Part (i) of Theorem 2.1 yields $\rho_{V}\left(g_{\tau}\right) F(i, v, p, \lambda)=y^{\frac{b_{+}}{4}+\frac{m_{+}}{2}+\frac{b_{-}}{4}+\frac{m_{-}}{2}} F(\tau, v, p, \lambda)$. On the other hand, after extending the Weil representation $\rho_{V}$ to the Lie algebra $\mathfrak{s l}_{2}(\mathbb{R})$ of $M p_{2}(\mathbb{R})$ and then to its universal enveloping algebra, Lemma 1.4 of [25] (with $m=2 k$ ) and part (ii) of Theorem 2.1 show that

$$
\rho_{V}(C) y^{\frac{k}{2}+\frac{b_{-}}{2}+m_{-}} F(\tau, v, p, \lambda)=4 \widetilde{\Delta}_{\frac{k}{2},-\frac{k}{2}, \tau} y^{\frac{k}{2}+\frac{b_{-}}{2}+m_{-}} F(\tau, v, p, \lambda),
$$

where $C=2 E F+2 F E+H^{2}$ is the Casimir element of the universal enveloping algebra of $\mathfrak{s l}_{2}(\mathbb{R})$. Now, the right hand side of Eq. (4) can be written as $y^{\frac{k}{2}}\left(4 \Delta_{k, \tau}+k(k-\right.$ 2)) $y^{\frac{b_{-}}{2}+m_{-}} F(\tau, v, p, \lambda)$. Moreover, Lemma 1.5 of [25] shows that the action of $\rho_{V}(C)$ coincides with that of $\Delta_{S_{b_{+}, b_{-}}^{+}}+\frac{\left(b_{+}+b_{-}\right)\left(b_{+}+b_{-}-4\right)}{4}$. Substituting the value of $k$ in the constant completes the proof of the proposition.

In the case $p=1$, Proposition 2.2 suffices to prove Proposition 4.5 of [6]. Indeed, in this case the action of $\mathrm{SO}_{b_{+}, b_{-}}^{+}$in the $\lambda$ variable coincides with its action on the $v$ variable. Thus the actions of the Laplacians coming from these two operations of $\mathrm{SO}_{b_{+}, b_{-}}^{+}$coincide, so that one only needs to find the form of $\Delta_{\mathrm{SO}_{b_{+}, b_{-}}^{+}}$in the variable of the Grassmannian $G(V)$. Here we consider the case of a non-trivial polynomial. There is thus no evident connection between these two actions (and Laplacians). Indeed, $p$ depends on $v$ by the homogeneity condition, and this dependence affects strongly the form which $\Delta_{\mathrm{SO}_{b_{+}, b_{-}}^{+}, v}$ attains. However, we now present a particular case in which the actions can be related.

\subsection{Grassmannians with complex structures}

We take $b_{+}=2$, where the Grassmannian carries the structure of a complex manifold, which we now briefly describe (see Section 13 of [2] or Section 3.2 of [6] for more details). Indeed, in this case $G(V)$ is diffeomorphic to a connected component of the analytically open subset which is defined by the inequality $\left(Z_{V}, \overline{Z_{V}}\right)>0$ on the conic $\mathbb{P}\left(V_{\mathbb{C}}\right)_{0}=$ $\left\{\left[Z_{V}\right] \in \mathbb{P}\left(V_{\mathbb{C}}\right) \mid Z_{V}^{2}=0\right\}$ (here $\mathbb{P}$ stands for the associated projective space). The inverse image $P$ of this variety in $V_{\mathbb{C}}$ corresponds, by taking the real and imaginary parts of a complex vector, to the set of oriented, orthogonal pairs of vectors of the same positive 
norm. Such a pair of vectors forms a basis for $v_{+}$in the image of that point in $G(V)$. We may view $P$ as a $\mathbb{C}^{*}$-bundle over $G(V)$.

Given an indefinite vector space $V$, pick an isotropic vector $z \in V$. Then $K_{\mathbb{R}}=z^{\perp} / \mathbb{R} z$ is non-degenerate of signature $\left(b_{+}-1, b_{-}-1\right)$. By choosing $\zeta \in V$ with $(z, \zeta)=1$, we identify $K_{\mathbb{R}}$ with $\{z, \zeta\}^{\perp}$. Vectors of $V$ may then be written as triplets $(\eta, a, b)$, with $\eta \in K_{\mathbb{R}}$ and $a$ and $b$ in $\mathbb{R}$. This symbol stands for the vector $\eta+a \zeta+b z$ (under the identification of $K_{\mathbb{R}}$ with $\{z, \zeta\}^{\perp}$ ), which has norm $\eta^{2}+a^{2} \zeta^{2}+2 a b$. If $b_{+}=2$ then the choice of $z$ defines a holomorphic section $G(V) \rightarrow P$ by picking the element pairing to 1 with $z$. Moreover, $K_{\mathbb{R}}$ is then Lorentzian, so that the set of positive norm vectors is the disjoint union of two cones. The choice of $z$ and of the orientation on the bases determine one cone $C$ to be the positive one. Our section then yields a biholomorphic diffeomorphism between $G(V)$ and the tube domain $K_{\mathbb{R}}+i C$. Under this identification, the section maps $Z=X+i Y \in K_{\mathbb{R}}+i C$ to the element $Z_{v, V}=\left(Z, 1, \frac{-Z^{2}-\zeta^{2}}{2}\right)$ of $P$ (the inverse map subtracts $\zeta$ and projects to $K_{\mathbb{C}}$ ). The real and imaginary parts of this vector are $X_{v, V}=\left(X, 1, \frac{Y^{2}-X^{2}-\zeta^{2}}{2}\right)$ and $Y_{v, V}=(Y, 0,-(X, Y))$ respectively. We have $X_{v, V} \perp Y_{v, V}$ and $X_{v, V}^{2}=X_{v, V}^{2}=Y^{2}>0$. Here $v$ stands for the element of $G\left(L_{\mathbb{R}}\right)$ in which $v_{+}$is spanned by the latter two vectors.

For non-negative integers $r, s$, and $t$, let $P_{r, s, t}(Z, \lambda)=\frac{\left(\lambda, Z_{v, V}\right)^{r}\left(\lambda, \overline{Z_{v, V}}\right)^{t}}{\left(Y^{2}\right)^{s}}$. It is homogenous of degree $(r+t, 0)$ with respect to $v$. Hence the constant in Proposition 2.2 reduces, for $p=P_{r, s, t}$, to $(r+t)\left(r+t-b_{-}\right)$. Given an even lattice $L$ in $V$, we denote the theta function $\Theta_{L}\left(\tau, v, P_{r, s, t}\right)$ by $\Theta_{L, r, s, t}(\tau, v)$.

Let $O^{+}(V)$ be the subgroup of $O(V)$ in which the orientation on the positive definite part is preserved. It operates on $G(V) \cong K_{\mathbb{R}}+i C$, with a factor of automorphy which we denote $j(\sigma, Z)$ for $\sigma \in O^{+}(V)$ and $Z \in K_{\mathbb{R}}+i C$. It is defined by the equation

$$
\sigma\left(Z_{v, V}\right)=j(\sigma, Z) Z_{\sigma v, V}, \quad \text { or equivalently } j(\sigma, Z)=\left(\sigma\left(Z_{v, V}\right), z\right) .
$$

Given integers $k$ and $l$ and a discrete subgroup $\Gamma$ of $O^{+}(V)$, we say that a function $\Phi: K_{\mathbb{R}}+i C \rightarrow \mathbb{C}$ (or on $G(V)$ ) is an automorphic form of weight $(k, l)$ with respect to $\Gamma$ if the equality

$$
\Phi(\sigma Z)=j(\sigma, Z)^{k} \overline{j(\sigma, Z)}^{l} \Phi(Z)
$$

holds for every $Z \in K_{\mathbb{R}}+i C$ and $\sigma \in \Gamma$. A standard argument shows that this is equivalent to the definition of automorphy appearing in [2], using homogeneity and $\Gamma$-invariance on $P$. We now prove

Proposition 2.3. For fixed $\tau \in \mathcal{H}$, the function $v \mapsto \Theta_{L, r, s, t}(\tau, v)$ is automorphic of weight $(s-r, s-t)$ with respect to the discriminant group $\Gamma$ of $L$, namely the kernel of the natural map from Aut ${ }^{+}(L)=\operatorname{Aut}(L) \cap O^{+}(L)$ to $\operatorname{Aut}\left(L^{*} / L\right)$.

Proof. The definition of $j(\sigma, Z)$ and the equality $(\Im(\sigma Z))^{2}=\frac{Y^{2}}{|j(\sigma, Z)|^{2}}$ from (the simple) Lemma 3.20 of [6] imply the equality

$$
P_{r, s, t}(\sigma Z, \lambda)=j(\sigma, Z)^{s-r} \overline{j(\sigma, Z)}^{s-t} P_{r, s, t}\left(Z, \sigma^{-1} \lambda\right)
$$

for all $Z \in K_{\mathbb{R}}+i C, \lambda \in V$, and $\sigma \in O^{+}(V)$. Now, $\Delta_{v} P_{r, s, t}=\Delta_{v_{+}} P_{r, s, t}$ is just $4 r t P_{r-1, s-1, t-1}$. As this operation preserves the differences $s-r$ and $s-t$, Eq. (5) continues to hold if we replace $P_{r, s, t}$ by $e^{-\Delta_{v} / 8 \pi y} P_{r, s, t}$. By the usual automorphic properties of the expression 
$\mathbf{e}\left(\tau \frac{\lambda_{v_{+}}^{2}}{2}+\bar{\tau} \frac{\lambda_{v_{-}}^{2}}{2}\right)$, the validity of Eq. (5) extends also to $F_{r, s, t}(\tau, Z, \lambda)=F\left(\tau, v, P_{r, s, t}, \lambda\right)$. Now, as the operation of $\sigma \in \Gamma$ preserves absolutely convergent sums on cosets of $L$ inside $L^{*}$, this completes the proof of the proposition.

For $\sigma \in O^{+}(V)$, let $[\sigma]_{s-r, s-t}$ denote the slash operator of weight $(k, l)$ :

$$
\Phi[\sigma]_{k, l}(Z)=\Phi(\sigma Z) j(\sigma, Z)^{-k} \overline{j(\sigma, Z)}^{-l}
$$

Equation (5) relates the usual action of $O^{+}(V)$ on the $\lambda$ variable in $P_{r, s, t}$ or $F_{r, s, t}$ to its action on $Z$ via the slash operators of weight $(s-r, s-t)$. As the operator $\Delta_{S O_{b_{+}, b_{-}}^{+}, \lambda}$ has order 2 and it commutes with the action of $\mathrm{SO}_{b_{+}, b_{-}}^{+}$on $\lambda$, its action must be related to an operator (of order 2) in the $Z$ variable which commutes with the action of these slash operators. The standard theory of Laplacians and Casimir operators in simple Lie groups shows that there should be only one such second order differential operator (up to multiplicative and additive constants). Let $\Delta^{G}$ be the operator which in an orthonormal basis for $K_{\mathbb{R}}$ takes the form

$$
8 \sum_{g, h} y_{g} y_{h} \partial_{g} \bar{\partial}_{h}-4 Y^{2}\left(\partial_{1} \bar{\partial}_{1}-\sum_{k>1} \partial_{k} \bar{\partial}_{k}\right)
$$

(this is 8 times the operator $\Delta_{1}$ of [21] or $8 \Omega$ in the notation of [6]), and define $D^{*}=$ $\sum_{h} y_{h} \partial_{h}$ and $\overline{D^{*}}=\sum_{h} y_{h} \bar{\partial}_{h}$. The differential operator we are looking for is given in the following

\section{Lemma 2.4. The combination}

$$
\widetilde{\Delta}_{k, l}^{G}=\Delta^{G}-4 i k \overline{D^{*}}+4 i l D^{*}
$$

commutes with the slash operators $[\sigma]_{k, l}$ for every $\sigma \in O^{+}(V)$.

Proof. [21] has shown that $O^{+}(V)$ is generated by the elements $p_{\xi}$ for $\xi \in K_{\mathbb{R}}, k_{a, A}$ for $a \in \mathbb{R}^{*}$ and $A \in O^{\operatorname{sgn}(a)}\left(K_{\mathbb{R}}\right)$, and $w$. In the $K_{\mathbb{R}}+\mathbb{R} \zeta+\mathbb{R} z$ notation, under the assumption $\zeta^{2}=0$ (which is made in [21] and can always be satisfied by replacing $\zeta$ by $\zeta-\frac{\zeta^{2}}{2} z$ ), these elements take the form

$$
p_{\xi}=\left(\begin{array}{ccc}
I & 0 & -\xi^{*} \\
\xi & 1 & -\frac{\xi^{2}}{2} \\
0 & 0 & 1
\end{array}\right), \quad k_{a, A}=\left(\begin{array}{ccc}
A & 0 & 0 \\
0 & \frac{1}{a} & 0 \\
0 & 0 & a
\end{array}\right), \quad w=\left(\begin{array}{ccc}
\tilde{w} & 0 & 0 \\
0 & 0 & -1 \\
0 & -1 & 0
\end{array}\right),
$$

where $\xi^{*}: K_{\mathbb{R}} \rightarrow \mathbb{R}$ is defined by pairing with $\xi$ and $\widetilde{w}$ is the reflection with respect to the hyperplane perpendicular to a pre-fixed positive norm vector in $K_{\mathbb{R}}$. The sign condition on $A$ ensures that $k_{a, A} \in O^{+}(V)$, and $w$ lies in $S O^{+}(V)$. Hence it suffices to verify the commutativity of $\widetilde{\Delta}_{k, l}^{G}$ with $[\sigma]_{k, l}$ for $\sigma$ being one of the elements $p_{\xi}, k_{a, A}$, or $w$.

For $\sigma=p_{\xi}$ and for $\sigma=k_{a, A}$ the automorphy factor $j(\sigma, Z)$ is a constant function of $Z$ ( 1 for $p_{\xi}, \frac{1}{a}$ for $k_{a, A}$ ). Hence the assertion follows easily from the fact that the three operators $\Delta^{G}, D^{*}$, and $\overline{D^{*}}$ are invariant under such $\sigma$. For $\sigma=w$, the action of the part $-4 i k \overline{D^{*}}+4 i l D^{*}$ of $\widetilde{\Delta}_{k, l}^{G}$ on $F[\sigma]_{k, l}$ yields

$$
-4 i k\left[\overline{D^{*}}(F \circ w) \bar{j}^{-l}+(F \circ w){\overline{D^{*} j}}^{-l}\right] j^{-k}+4 i l\left[D^{*}(F \circ w) j^{-k}+(F \circ w) D^{*} j^{-k}\right] \bar{j}^{-l} \text {. }
$$


On the other hand, $\Delta^{G}$ is the sum of two operators, so that apart from the expression $\Delta^{G}(F \circ w) j^{-k_{j}^{-l}}$, the combination $\Delta^{G}\left(F[\sigma]_{k, l}\right)$ involves

$$
8 \overline{D^{*}}(F \circ w) D^{*} j^{-k} \bar{j}^{-l}+8 D^{*}(F \circ w) j^{-k}{\overline{D^{* j}}}^{-l}+8(F \circ w) D^{*} j^{-k}{\overline{D^{* j}}}^{-l}
$$

from the action of the operator $\sum_{g, h} y_{j} y_{h} \partial_{g} \bar{\partial}_{h}$ and similar three expressions from the action of the other operator. The automorphy factor $j(w, Z)$ is $\frac{Z^{2}}{2}$. Evaluating $D^{*} j^{-k}$, $\overline{D^{*}} \bar{j}^{-l}$, and the other derivatives of $j^{k}$ and $\bar{j}^{-l}$ thus shows that $\widetilde{\Delta}_{k, l}^{G}\left(F[\sigma]_{k, l}\right)$ equals

$$
\begin{aligned}
& \Delta^{G}(F \circ w) j^{-k} \bar{j}^{-l}-2 i k \bar{Z}^{2} \overline{D^{*}}(F \circ w) j^{-k-1} \bar{j}^{-l}+2 i l Z^{2} D^{*}(F \circ w) j^{-k} \bar{j}^{-l-1}+ \\
& +4 k Y^{2} \bar{D}(F \circ w) j^{-k-1} \bar{j}^{-l}+4 l Y^{2} D(F \circ w) j^{-k} \bar{j}^{-l-1}
\end{aligned}
$$

(and the coefficients of $F(w Z) j^{-k-1} \bar{j}^{-l-1}$ cancel out). Now, the Theorem of [21] shows that $\Delta^{G}(F \circ w)(Z)=\left(\Delta^{G} F\right)(w Z)$, and the formulae concerning $D^{*}$ and $\overline{D^{*}}$ in [21] translate to

$$
D^{*}(F \circ w)(Z)=\frac{W^{2}}{\bar{W}^{2}}\left(D^{*} F\right)(w Z)-2 i \frac{(\Im W)^{2}}{\bar{W}^{2}}(D F)(w Z)
$$

and

$$
\overline{D^{*}}(F \circ w)(Z)=\frac{\bar{W}^{2}}{W^{2}}\left(\overline{D^{*}} F\right)(w Z)+2 i \frac{(\Im W)^{2}}{W^{2}}(\bar{D} F)(w Z),
$$

with $W=w(Z)$ (recall that the expressions denoted $\delta$ and $d$ in [21] are $\frac{Z^{2}}{2}$ and $\frac{Y^{2}}{2}$ respectively). One also verifies that $D(F \circ w)(Z)=-(D F)(w Z)$ and $\bar{D}(F \circ w)(Z)=-(\bar{D} F)(w Z)$, while $\frac{W^{2}}{2}=\frac{2}{Z^{2}}, \frac{\bar{W}^{2}}{2}=\frac{2}{\bar{Z}^{2}}$, and $(\Im W)^{2}=\frac{4 Y^{2}}{Z^{2} \bar{Z}^{2}}$. Therefore, $\widetilde{\Delta}_{k, l}^{G}\left(F[\sigma]_{k, l}\right)(Z)$ equals

$$
\left(\Delta^{G} F\right)(w Z) j^{-k_{j}^{-l}}-4 i k \overline{D^{*}} F(w Z) j^{-k} \bar{j}^{-l}+4 i l D^{*} F(w Z) j^{-k_{j}^{-l}},
$$

which agrees with the value $\left(\widetilde{\Delta}_{k, l}^{G} F\right)[\sigma]_{k, l}(Z)$. This proves the lemma.

The weight $(k, l)$ Laplacian on $G(V)$ is the operator $\Delta_{k, l}^{G}=\widetilde{\Delta}_{k, l}^{G}-2\left(2 k-b_{-}\right) l$. Lemma 3.20 of [6] shows that multiplication by $\left(Y^{2}\right)^{t}$ takes an automorphic form of weight $(k+$ $t, l+t)$ to an automorphic form of weight $(k, l)$. One also verifies that the operators $\Delta_{k, l}^{G}$ satisfy the relation $\Delta_{k, l}^{G}\left(Y^{2}\right)^{t}=\left(Y^{2}\right)^{t} \Delta_{k+t, l+t}^{G}$.

We remark that the results of [21] are stated for $b_{-}$(or $q$ in the notation of [21]) being at least 3. However, the proof holds equally well for $q=2$, and the same applies for our Lemma 2.4. For $b_{-}=1$ the Grassmannian $K_{\mathbb{R}}+i C$ is $\mathcal{H}$, the operator $\Delta^{G}$ is the usual Laplacian $4 y^{2} \partial \bar{\partial}$ on $\mathcal{H}$, and $\Delta_{k, l}^{G}$ is $\Delta_{2 k, 2 l}$. Therefore, Lemma 2.4 holds for any value of $b_{-}$.

\subsection{A differential equation for $\Theta_{L, r, s, t}$}

The following generalization of Proposition 4.5 of [6] will turn out very important for our purposes:

Proposition 2.5. Let $L$ be an even lattice in the space $V$ of signature $\left(2, b_{-}\right)$, and let $k=1+\frac{b_{-}}{2}+r+t$. Then the theta function $\Theta_{L, r, s, t}$ satisfies the differential equation

$$
4 \Delta_{k, \tau} y^{\frac{b_{-}}{2}} \Theta_{L, r, s, t}(\tau, Z)=\left[\Delta_{s-r, s-t, Z}^{G}+2 r\left(b_{-}-2 t\right)\right] y^{\frac{b_{-}}{2}} \Theta_{L, r, s, t}(\tau, Z) .
$$

Proof. We follow the proof of Proposition 4.5 of [6], with the necessary adjustments. It suffices to prove that the basic summand $F_{r, s, t}(\tau, Z, \lambda)$ satisfies this differential equation for any $\lambda$. Let $\rho_{k, l}$ be the representation of $O^{+}(V)$ on $C^{\infty}(G(V))$ using the weight $(k, l)$ 
slash operators, namely $\rho_{k, l}(\sigma) \Phi=\Phi\left[\sigma^{-1}\right]_{k, l}$, and extend it to the universal enveloping algebra of $\mathfrak{s o}(V)$. Lemma 2.4 shows that the action of the Casimir operator of $O^{+}(V)$ via $\rho_{k, l}$ must be the same as that of $\alpha \Delta_{k, l}^{G}+\beta$ for some constants $\alpha$ and $\beta$. Eq. (5) and the paragraph following it imply the equality

$$
F_{r, s, t}\left(\tau, Z, \sigma^{-1} \lambda\right)=\rho_{s-r, s-t}(\sigma) F_{r, s, t}(\tau, Z, \lambda)
$$

for every $\tau \in \mathcal{H}, Z \in K_{\mathbb{R}}+i C, \lambda \in V$, and $\sigma \in O^{+}(V)$. Since the Casimir operator of $\mathrm{O}^{+}(V)$ acts on functions of $\lambda$ as $\Delta_{S O_{2, b_{-}}^{+}}$, we obtain

$$
\Delta_{S O_{2, b_{-}}^{+}, \lambda} F_{r, s, t}(\tau, Z, \lambda)= \pm\left(\alpha \Delta_{s-r, s-t}^{G}+\beta\right) F_{r, s, t}(\tau, Z, \lambda) .
$$

Proposition 2.2 now yields

$$
4 \Delta_{k, \tau} y^{\frac{b_{-}}{2}} F_{r, s, t}(\tau, Z, \lambda)=\left(\widetilde{\alpha} \Delta_{s-r, s-t}^{G}+\widetilde{\beta}\right) y^{\frac{b_{-}}{2}} F_{r, s, t}(\tau, Z, \lambda)
$$

for some constants $\widetilde{\alpha}$ and $\widetilde{\beta}$ which are independent of $\lambda$. Choose a basis for $K_{\mathbb{R}}$ in which the first two basis elements span a hyperbolic plane and the rest are orthonormal (or with common norm -2 , as in [6] and [21]), and take $\lambda$ to be the second basis element. Then evaluating $e^{-\Delta_{v} / 8 \pi y}\left(P_{r, s, t}\right)$ shows that

$$
F_{r, s, t}(\tau, Z, \lambda)=\sum_{j=0}^{\min \{r, t\}} \frac{j !}{(-2 \pi)^{j}}\left(\begin{array}{l}
r \\
j
\end{array}\right)\left(\begin{array}{l}
t \\
j
\end{array}\right) \frac{z_{1}^{r-j} \overline{z_{1}} t-j}{\left(Y^{2}\right)^{s-j}} e^{-2 \pi y \frac{\left|z_{1}\right|^{2}}{Y^{2}}},
$$

with $z_{1}$ being the first coordinate of $Z$ in this basis. A straightforward computation shows that $\widetilde{\alpha}=1$ and $\widetilde{\beta}=2 r\left(b_{-}-2 t\right)$. This proves the proposition.

We remark that all these results extend to the case where $P_{r, s, t}$ is multiplied by $\left(\lambda_{v_{-}}^{2}\right)^{h}$ for some integer $h$ (powers of $\lambda_{v_{+}}^{2}$ just change the indices of $P_{r, s, t}$, hence produce no new functions). Then the weight is $k=1+\frac{b_{-}}{2}+r+t-2 h$, and the constant $\tilde{\beta}$ from Proposition 2.5 becomes $2(r-h)\left(b_{-}-2(t-h)\right)$. However, we shall not need this generalization in this paper.

In fact, as we are concerned with explicit functions and operators, Eq. (4) and Propositions 2.2 and 2.5 can be obtained by direct evaluation of the corresponding derivatives. In addition, it is expedient to evaluate the images of $y^{\frac{b_{-}}{2}+m_{-}} F(\tau, v, p, \lambda)$ under the weight raising operator $R_{k}=2 i \frac{\partial}{\partial \tau}+\frac{k}{y}$ and the weight lowering operator $L=-2 i y^{2} \frac{\partial}{\partial \bar{\tau}}$ (note the sign difference relative to [6]!) in general. The resulting functions are

$$
-2 \pi y^{\frac{b_{-}}{2}+m_{-}} F\left(\tau, v, \lambda_{v_{+}}^{2} p, \lambda\right)-\frac{1}{8 \pi} y^{\frac{b_{-}}{2}+m_{-}-2} F\left(\tau, v, \Delta_{v_{-}} p, \lambda\right)
$$

and

$$
2 \pi y^{2+\frac{b_{-}}{2}+m_{-}} F\left(\tau, v, \lambda_{v_{-}}^{2} p, \lambda\right)+\frac{1}{8 \pi} y^{\frac{b_{-}}{2}+m_{-}} F\left(\tau, v, \Delta_{v_{+}} p, \lambda\right)
$$

respectively. Thus similar formulae describe the action of these operators on arbitrary theta functions with polynomials. One verifies that the action of $\Delta_{S O_{b_{+}, b_{-}}^{+}, \lambda}$ on $y^{\frac{b_{-}}{2}+m_{-}} F(\tau, v, p, \lambda)$ takes it to $y^{\frac{b_{-}}{2}+m_{-}} q(\lambda) \mathbf{e}\left(\tau \frac{\lambda_{v_{+}}^{2}}{2}+\bar{\tau} \frac{\lambda_{\nu_{-}}^{2}}{2}\right)$ with $q$ being the image of $e^{-\Delta_{v} / 8 \pi y}(p)$ under the operator

$$
\Delta_{S O_{b_{+}, b_{-}}^{+}}+8 \pi y \lambda_{v_{-}}^{2} I_{v_{+}}-8 \pi y \lambda_{v_{+}}^{2} I_{v_{-}}+4 \pi y b_{+} \lambda_{v_{-}}^{2}-4 \pi y b_{-} \lambda_{v_{+}}^{2}-16 \pi^{2} y^{2} \lambda_{v_{+}}^{2} \lambda_{v_{-}}^{2} .
$$


The symbol $I_{U}$, for a vector space $U$, stands for the homogeneity operator $\sum_{j} u_{j} \frac{\partial}{\partial u_{j}}$ in some (hence any) basis $\left(u_{j}\right)_{j=1}^{\operatorname{dim} U}$ of $U$. Evaluating the action of $\Delta_{k}=R_{k-2} \cdot L$ for $k=$ $\frac{b_{+}}{2}+m_{+}-\frac{b_{-}}{2}-m_{-}$on $y^{\frac{b_{-}}{2}+m_{-}} F(\tau, v, p, \lambda)$ yields the function described in Eq. (7) up to the constant multiple of $y^{\frac{b_{-}}{2}+m_{-}} F(\tau, v, p, \lambda)$ appearing in Proposition 2.2. In the case $b_{+}=2$ and the polynomial $P_{r, s, t}$, one can check directly that the operator from Eq. (7) takes $P_{r, s, t}$ to

$$
-4 \lambda_{-}^{2} a_{r, t} P_{r-1, s-1, t-1}-4 \pi y b_{-} P_{r+1, s+1, t+1}+2 t b_{-} P_{r, s, t},
$$

where $a_{r, t}$ stands for

$$
\left(r-2 \pi y \lambda_{v_{+}}^{2}\right)\left(t-2 \pi y \lambda_{v_{+}}^{2}\right)-2 \pi y \lambda_{v_{+}}^{2}=r t-(r+t+1) 2 \pi y \lambda_{v_{+}}^{2}+\left(2 \pi y \lambda_{v_{+}}^{2}\right)^{2} .
$$

A direct evaluation of the action of the operator $\Delta_{s-r, s-t}^{G}+(r-t)^{2}+b_{-}(r-t)$ on $P_{r, s, t}(Z, \lambda) \mathbf{e}\left(\tau \frac{\lambda_{v_{+}}^{2}}{2}+\bar{\tau} \frac{\lambda_{v_{-}}^{2}}{2}\right)$ gives the expression from Eq. (8) multiplied by $\mathbf{e}\left(\tau \frac{\lambda_{v_{+}}^{2}}{2}+\bar{\tau} \frac{\lambda_{\nu_{-}}^{2}}{2}\right)$. Recall that $\Delta_{v_{+}}^{j} P_{r, s, t}$ is some constant multiple of $P_{r-j, s-j, t-j}$ for any $j \leq \min \{r, t\}$, and the difference $(r-j)-(t-j)$ equals $r-t$. It follows that $\Delta_{s-r, s-t}^{G}+(r-t)^{2}+b_{-}(r-t)$ and $\Delta_{S_{b_{+}, b_{-}, \lambda}^{+}}$give the same result also on $F_{r, s, t}$. This argument suggests an alternative proof of Eq. (4) and of Propositions 2.2 and 2.5 (as well as of Proposition 4.5 of [6]), a proof which is independent of Theorem 2.1, the results of [25], and theorems about actions of Casimir operators and Laplacians in general.

\section{Theta lifts with polynomials}

In this Section we evaluate the theta lifts of certain almost weakly holomorphic modular forms, which will give the main tool for the arithmetic application later (see Definitions 4.3 and 4.4 below). Many parts of this Section are very technical, and skipping most of it except the statements of Theorem 3.9 for general $b_{-}$and Theorem 3.10 for $b_{-}=1$ may suffice on the first reading.

\subsection{Modular forms and their theta lifts}

Let $\Gamma \subseteq M p_{2}(\mathbb{R})$ be a Fuchsian group of the first kind, and let $\rho$ be a representation of $\Gamma$ on some finite-dimensional complex vector space $V_{\rho}$. Given $k$ and $l$ in $\frac{1}{2} \mathbb{Z}$, a modular form of weight $(k, l)$ and representation $\rho$ with respect to $\Gamma$ is a real-analytic function $f: \mathcal{H} \rightarrow V_{\rho}$ which satisfies the functional equation

$$
f(M \tau)=j(M, \tau)^{k} \overline{j(M, \tau)}^{l} \rho(M) f(\tau)
$$

for every $\tau \in \mathcal{H}$ and $M \in \Gamma$ (the metaplectic data makes the half-integral powers well-defined). For example, part (iii) of Theorem 2.1 states that $\Theta_{L}$ is modular of weight $\left(\frac{b_{+}}{2}+m_{+}, \frac{b_{-}}{2}+m_{-}\right)$and representation $\rho_{L}$ with respect to $M p_{2}(\mathbb{Z})$. A function $f: \mathcal{H} \rightarrow V_{\rho}$ is called almost holomorphic if it takes the form $f(\tau)=\sum_{k=0}^{k_{\max }} \frac{f_{k}(\tau)}{y^{k}}$, for some integer $k_{\text {max }}$, with the $f_{k}$ holomorphic functions. This condition is stable under the action of $M p_{2}(\mathbb{R})$. In case $\Gamma$ has cusps, a weakly holomorphic modular form is a modular form which is holomorphic on $\mathcal{H}$ but may have poles at the cusps. Relaxing the requirement of holomorphicity on $\mathcal{H}$ to almost holomorphicity yields almost weakly holomorphic modular forms. The weight raising operator $R_{k}$ increases the weight of a modular form of weight $k$ by 2 , while the weight lowering operator $L$ reduces it by 2 . Both preserve almost 
holomorphicity and almost weak holomorphicity, with the latter operator annihilating (weakly) holomorphic modular forms.

A modular form $f$ of any weight $(k, l)$ and representation $\rho_{L}$ with respect to $M p_{2}(\mathbb{Z})$ has a Fourier expansion

$$
f(\tau)=\sum_{\gamma \in L^{*} / L} \sum_{n \in \mathbb{Q}} c_{\gamma, n}(y) q^{n} e_{\gamma}
$$

with the standard notation $q=\mathbf{e}(\tau)$. Here $c_{\gamma, n}$ are smooth functions of $y$ which vanish unless $n \in \mathbb{Z}+\frac{\gamma^{2}}{2}$. If $f$ is almost weakly holomorphic then the functions $c_{\gamma, n}$ are polynomials of bounded degree in $\frac{1}{y}$, and if $f$ is weakly holomorphic then they are constants. In both cases they vanish unless $n \gg-\infty$.

Let $L$ be an even lattice of signature $\left(b_{+}, b_{-}\right)$. We take, for every $v \in G\left(L_{\mathbb{R}}\right)$, a polynomial $p_{v}$ on $L_{\mathbb{R}}$ which is homogenous of degree $\left(m_{+}, m_{-}\right)$with respect to $v$. Assume that $p_{v}$ depends smoothly on $v$. Let $F$ be a (not necessarily holomorphic) modular form of weight $\frac{b_{+}}{2}+m_{+}-\frac{b_{-}}{2}-m_{-}$and representation $\rho_{L}$. Considering $\mathbb{C}\left[L^{*} / L\right]$ as a unitary space in which the canonical basis is orthonormal, the function $\tau \mapsto y^{\frac{b_{+}}{2}+m_{+}}\left\langle F(\tau), \Theta_{L}\left(\tau, v, p_{v}\right)\right\rangle_{\rho_{L}}$ is $M p_{2}(\mathbb{Z})$-invariant for every $v$. Following [2] and others, we define the theta lift of $F$ as the integral

$$
\Phi_{L}\left(v, F, p_{v}\right)=\int_{X(1)} y^{\frac{b_{+}}{2}+m_{+}}\left\langle F(\tau), \Theta_{L}\left(\tau, v, p_{v}\right)\right\rangle_{\rho_{L}} \frac{d x d y}{y^{2}}
$$

as a function of $v \in G\left(L_{\mathbb{R}}\right)$. If $F$ decreases exponentially towards the cusp, then the integral in Eq. (10) converges for every such $v$, yielding a smooth function on $G\left(L_{\mathbb{R}}\right)$ (depending on the polynomial $p: v \mapsto p_{v}$ ). If $F$ grows exponentially toward the cusp, then the integral in Eq. (10) diverges, but can be regularized as follows. Let

$$
D=\{\tau \in \mathcal{H}|| \Re \tau|\leq 1 / 2,| \tau \mid \geq 1\}
$$

be the classical fundamental domain for $S L_{2}(\mathbb{Z})$, and let $D_{w}$ be the compact domain $\{\tau \in$ $D \mid y \leq w\}$ for every $w>1$. Multiply the integrand from Eq. (10) by $y^{-s}$, carry out the integration over $D_{w}$, and take the limit as $w \rightarrow \infty$. For $\lambda \in L^{*}$ with $\lambda^{2}<0$ we define the sub-Grassmannian

$$
\lambda^{\perp}=\left\{v \in G\left(L_{\mathbb{R}}\right) \mid \lambda \in v_{-}\right\} \subseteq G\left(L_{\mathbb{R}}\right)
$$

of $G\left(L_{\mathbb{R}}\right)$. It has real codimension $b_{+}$in $G\left(L_{\mathbb{R}}\right)$, and in the case $b_{+}=2$ it has complex codimension 1 there. The results of Sections 6 and 7 of [2] give the following

Theorem 3.1. Assume that the coefficients $c_{\gamma, n}(y)$ in Eq. (9) satisfy the condition that for any two real numbers $\alpha>0$ and $\beta \geq 0$, the integral $\int_{0}^{\infty} c_{\gamma, n}(y) e^{-\alpha / y-\beta y} y^{-t} d y$ converges for $t \in \mathbb{C}$ with $\Re t \gg-\infty$ to give a function of $t$ which can be meromorphically continued to all $t \in \mathbb{C}$. Then the limit from above produces, for any $v \in G\left(L_{\mathbb{R}}\right)$, the restriction of a meromorphic function of $s \in \mathbb{C}$ to some right half-plane in $\mathbb{C}$. The constant term of this function at $s=0$ yields a function on $G\left(L_{\mathbb{R}}\right)$, which is smooth outside a locally finite union of the sub-Grassmannians $\lambda^{\perp}$ defined above, and has singularities along these subGrassmannians.

Note that a function is considered "singular" along a submanifold if it is not smooth there, not only if it is discontinuous. 
Assume now that the modular form $F$ is almost weakly holomorphic, so that Eq. (9) becomes

$$
F(\tau)=\sum_{\gamma \in L^{*} / L} \sum_{k=0}^{k_{\max }} \sum_{n \gg-\infty} c_{\gamma, n, k} \frac{q^{n}}{y^{k}} e_{\gamma}
$$

for some integer $k_{\max }$ with $c_{\gamma, n, k} \in \mathbb{C}$. The polar part of $F$ is a finite sum, and we have

Theorem 3.2. An almost weakly holomorphic modular form satisfies the condition of Theorem 3.1. If such a modular form is expanded as above, then given $j$ and $k$ we define $\beta=\frac{b_{+}}{2}+m_{+}-j-k-1$. The singularity of the regularized theta lift $\Phi_{L}$ from Theorem 3.1 along $\lambda^{\perp}$ then looks like

$$
\sum_{\alpha \lambda \in L^{*}} \sum_{j, k} \frac{c_{\alpha \lambda, \frac{\alpha^{2} \lambda^{2}}{2}, k} \Delta_{\nu}^{j}\left(\overline{p_{v}}\right)(\alpha \lambda)}{(-8 \pi)^{j} j !}\left\{\begin{array}{cc}
\frac{\Gamma(\beta)}{\left(2 \pi \alpha^{2} \lambda_{v_{+}}^{2}\right)^{\beta}} & \beta \notin-\mathbb{N} \\
\frac{\ln \left(\alpha^{2} \lambda_{v_{+}}^{2}\right)}{\left(-2 \pi \alpha^{2} \lambda_{v_{+}}^{2}\right)^{\beta}(-\beta) !} & \beta \in-\mathbb{N} .
\end{array}\right.
$$

The set $\mathbb{N}$ is assumed to include 0 in Theorem 3.2.

Proof. The fact that $F$ satisfies the condition of Theorem 3.1 follows from Lemmas 7.2 and 7.3 of [2] (note the difference in conventions arising from the fact that in Eq. (9) the coefficients $c_{\gamma, n}(y)$ multiply $q^{n} e_{\gamma}$ while in [2] the notation $c_{\gamma, n}(y)$ is used for the coefficient of $\left.\mathbf{e}(n x) e_{\gamma}\right)$. The form of the singularity is now given in Theorem 6.2 of [2]. This proves the theorem.

The sums in Theorem 3.2 are essentially finite: For $\alpha$ we consider only coefficients from the (finite) polar part of $F$, for $j$ we need $\Delta_{v}^{j}\left(\overline{p_{v}}\right)$ not to vanish, and $0 \leq k \leq k_{\max }$.

If $p_{v}$ is homogenous of degree $\left(m_{+}, m_{-}\right)$with respect to $v$ and $\sigma \in O^{+}(V)$ then $p_{v} \circ \sigma^{-1}$ has the same homogeneity degree with respect to $\sigma v$. Moreover, one has $\Delta_{\sigma v}\left(p \circ \sigma^{-1}\right)=$ $\left(\Delta_{\nu} p\right) \circ \sigma^{-1}$. Section 6 of [2] states that the equality

$$
\Phi_{L}\left(\sigma v, F, p_{\nu} \circ \sigma^{-1}\right)=\Phi_{L}\left(v, f, p_{v}\right)
$$

holds wherever the regularized theta lift of $F$ from Theorem 3.1 is well-defined and $\sigma$ lies in the discriminant kernel $\Gamma$ from Proposition 2.3. For $p=1$ this gives the $\Gamma$-invariance of $\Phi_{L}$. For a general polynomial $p_{v}$ the automorphic property of $\Phi_{L}$ depends on the behavior of $v \mapsto p_{v}$ under $\Gamma$. The latter may be complicated in general, but for the case $b_{+}=2$ and the polynomials $P_{r, s, t}$ defined above (or even for $\left.P_{r, s, t}\left(\lambda_{v_{-}}^{2}\right)^{h}\right)$, Proposition 2.3 implies

$$
\Phi_{L, r, s, t}(\sigma v, F)=j(\sigma, Z)^{s-t} \overline{j(\sigma, Z)}^{s-r} \Phi_{L, r, s, t}(v, F)
$$

for any $Z \in K_{\mathbb{R}}+i C$ and $\sigma \in \Gamma$ (the complex conjugation on $\Theta_{L}$ in $\Phi_{L}$ interchanges the powers of $j$ and $\bar{j})$. Here and throughout, $\Phi_{L, r, s, t}(v, F)$ denotes $\Phi_{L}\left(v, F, P_{r, s, t}\right)$.

At this point we need to sort out some inaccuracies in the proofs of [2] which are relevant for our discussion. In particular, the argument based on Lemma 14.1 of this reference (specifically Corollary 6.3 and Corollary 14.2 there) can be replaced by the following

Lemma 3.3. Let $C$ be a positive integer, and let $p$ be a polynomial of degree smaller than C. Then $\sum_{j=0}^{C}(-1)^{j}\left(\begin{array}{l}C \\ j\end{array}\right) p(j)=0$. 
Proof. Let $\left(\begin{array}{l}x \\ r\end{array}\right)$ is the polynomial $\prod_{i=0}^{r-1}(x-i) / r$ !, of degree $r$. Since we can write $p(x)$ as $\sum_{r=0}^{\operatorname{deg} p} a_{r}\left(\begin{array}{l}x \\ r\end{array}\right)$, it suffices to prove the claim for $p(x)=\left(\begin{array}{l}x \\ r\end{array}\right)$ with $0 \leq r<C$. In this case only terms with $j \geq r$ contribute, and the contribution is given by $\left(\begin{array}{l}C \\ j\end{array}\right)\left(\begin{array}{l}j \\ r\end{array}\right)=\left(\begin{array}{l}C \\ r\end{array}\right)\left(\begin{array}{l}C-r \\ j-r\end{array}\right)$. Hence we find that

$$
\sum_{j=r}^{C}(-1)^{j}\left(\begin{array}{l}
C \\
j
\end{array}\right)\left(\begin{array}{l}
j \\
r
\end{array}\right)=(-1)^{r}\left(\begin{array}{l}
C \\
r
\end{array}\right) \sum_{i=0}^{C-r}(-1)^{i}\left(\begin{array}{c}
C-r \\
i
\end{array}\right)=0
$$

as $r<C$. This proves the lemma.

Indeed, both Corollary 6.3 and Corollary 14.2 of [2] involve sums over binomial coefficients of the form $\left(\begin{array}{c}A+D-2 j \\ A-2 j\end{array}\right)=\left(\begin{array}{c}A+D-2 j \\ D\end{array}\right)$ for $A$ and $D$ non-negative integers with $D<C$ which are independent of $j$. This expression is a polynomial function of $j$ of degree $D<C$, so that our Lemma 3.3 applies for both cases.

Somewhat more disturbing are the assertions in Corollary 6.3 and Theorem 10.3 of this reference which state that certain expressions are polynomials in an oriented norm 1 vector $v_{1}$ in a Lorentzian space. Indeed, in our notation (with $v \mapsto p_{v}$ ), the "wall crossing formula" from Corollary 6.3 states that the difference between the values of the theta lift on two adjacent Weyl chambers $W$ and $\widetilde{W}$ with separating "wall" $\lambda^{\perp}$ for $(\lambda, W)>0$ is

$$
\sum_{x \lambda \in L^{*}} \sum_{j, k} \frac{4 c_{x \lambda, \frac{x^{2} \lambda^{2}}{2}, k} \Delta_{v}^{j}\left(\overline{p_{v}}\right)(x \lambda) \Gamma\left(m_{+}-j-k-\frac{1}{2}\right)}{(-8 \pi)^{j} j !}\left(\sqrt{2 \pi} x\left(\lambda, v_{1}\right)\right)^{1+2 j+2 k-2 m_{+}},
$$

with only $x>0$ and with $v_{1}$ being an oriented norm 1 vector spanning $v_{+}$. The proof there shows that only positive powers of $\left(\lambda, v_{1}\right)$ appear. However, this is not necessarily a polynomial in $v_{1}$ because of the unknown dependence of $p_{v}$ on $v$ (or on $v_{1}$ ). The fact that in [2] one does not work with $v \in G(M)$ but with $v$ an isometry from $L_{\mathbb{R}}$ to $\mathbb{R}^{b_{+}, b_{-}}$ does not overcome this problem, since then the expression in Eq. (12) depends on $v$ as an isometry and not only on its image in $G\left(L_{\mathbb{R}}\right)$. Hence this expression cannot be described as a function of $v_{1}$ alone. Only the smoothness of this difference as a function on all of $G\left(L_{\mathbb{R}}\right)$ survives (at least in our conventions with $\left.p_{v}\right)$. On the other hand, when $p_{v}(\lambda)=\left(\lambda, v_{1}\right)^{m_{+}}$ of homogeneity degree $\left(m_{+}, 0\right)$ (and no multiplying constant), the expression in Eq. (12) is indeed the restriction of a polynomial on $L_{\mathbb{R}}$ of the asserted degree to the set of vectors of the form $v_{1}$.

The decomposition of $p$ appearing just above Lemma 5.1 of [2] takes, in our notation, the following form. Given $v \in G\left(L_{\mathbb{R}}\right)$, a polynomial $p_{v}$ with the usual homogeneity property, and natural numbers $h_{+}$and $h_{-}$, there is a (unique) polynomial $p_{v, h_{+}, h_{-}}$on $K_{\mathbb{R}}$ such that the equality

$$
p_{\nu}(\lambda)=\sum_{h_{+}, h_{-}}\left(\lambda, z_{v_{+}}\right)^{h_{+}}\left(\lambda, z_{v_{-}}\right)^{h_{-}} p_{v_{,} h_{+}, h_{-}}\left[\left(\lambda-\frac{(\lambda, z) z_{v_{+}}}{z_{v_{+}}^{2}}\right) / \mathbb{R} z\right]
$$

holds for every $\lambda \in L_{\mathbb{R}} \cdot p_{v, h_{+}, h_{-}}$is homogenous of degree $\left(m_{+}-h_{+}, m_{-}-h_{-}\right)$with respect to the decomposition $w$ of $K_{\mathbb{R}}$ into the images $w_{ \pm}$of $z_{v_{ \pm}}^{\perp} \subseteq v_{ \pm}$in $K_{\mathbb{R}}$. Note that $p_{v, h_{+}, h_{-}}$depends on $v$ (not only on $w$ ), and the map $v \mapsto w$ is not injective. The map $\lambda$ $\mapsto\left(\lambda-\frac{(\lambda, z) z_{v_{+}}}{z_{v_{+}}^{2}}\right) / \mathbb{R} z$ corresponds to the map denoted $w$ in [2]. A careful verification of Sections 5 and 7 of [2] shows that the reduction formula in Theorem 7.1 of [2] holds in our notation with each $p_{w, h_{+}, h_{-}}$replaced by $p_{v, h_{+}, h_{-}}$. Now, Theorem 10.3 of [2] implies that the theta lift $\Phi_{L}$ is a smooth function inside any Weyl chamber. However, the assertion 
that it is a polynomial does not necessarily hold, because of the same problem discussed in the previous paragraph. Only in the special case in which $p_{v}(\lambda)=\left(\lambda, v_{1}\right)^{m_{+}}$, where we have $h_{-}=0$ and $p_{v, m_{+}, 0}=1$, the assertion of Theorem 10.3 of [2] holds as stated. This is related to the fact that the equality $p_{v} \circ \sigma^{-1}=p_{\sigma \nu}$ holds for this $p_{v}$, since $\Gamma$-invariance is used in the proof of that theorem.

These remarks show that one must be careful when investigating properties of lifts of modular forms using theta functions with polynomials. However, in the applications appearing in [2] one considers only the case $p=1$ (in Sections 11, 13, and 15 there), or some multiple of $\eta \mapsto\left(\eta, v_{1}\right)^{m_{+}}$(in Section 14). Hence the results of these sections hold as stated.

\subsection{Differential properties of certain theta lifts}

Let

$$
\delta_{l}=-\frac{R_{l}}{4 \pi}=\frac{\partial_{\tau}}{2 \pi i}-\frac{l}{4 \pi y}
$$

be the (normalized) weight raising operator for weight $l$, where $\partial_{\tau}$ denotes $\frac{\partial}{\partial \tau}$ from now on. A well-known formula for the composition of these operators, which is easily proved by induction, states that

$$
\delta_{l}^{m}=\delta_{l+2 m-2} \circ \ldots \circ \delta_{l}=\sum_{k}\left(\begin{array}{l}
m \\
k
\end{array}\right)\left[\prod_{s=m-k}^{m-1}(l+s)\right]\left(\frac{-1}{4 \pi y}\right)^{k}\left(\frac{\partial_{\tau}}{2 \pi i}\right)^{m-k}
$$

(see, for example, Equation (56) in [9]). Moreover, $\delta_{l}$ sends eigenfunctions of (minus) the weight $l$ Laplacian $\Delta_{l}$ having eigenvalue $\lambda$ to eigenfunctions of (minus) $\Delta_{l+2}$ with eigenvalue $\lambda+l$. Hence the $\delta_{l}^{m}$-images of the former functions have the eigenvalue $\lambda+m(l+m-1)$. In particular, if $f=\sum_{\gamma, n} c_{\gamma, n} q^{n} e_{\gamma}$ is weakly holomorphic of weight $1-\frac{b_{-}}{2}-m$ and representation $\rho_{L}$ for some even lattice $L$ of signature $\left(2, b_{-}\right)$then

$$
F=\delta_{1-\frac{b_{-}-m}{2}}^{m} f=\sum_{\gamma, n, k} c_{\gamma, n, k} \frac{q^{n}}{y^{k}} e_{\gamma}, \quad c_{\gamma, n, k}=\left(\begin{array}{c}
m \\
k
\end{array}\right) n^{m-k} \cdot \prod_{r=0}^{k-1}\left(r+\frac{b_{-}}{2}\right) \cdot \frac{c_{\gamma, n}}{(4 \pi)^{k}}
$$

is an almost weakly holomorphic modular form of weight $1-\frac{b_{-}}{2}+m$ and representation $\rho_{L}$ which is an eigenfunction of (minus) $\Delta_{1-\frac{b_{-}}{2}+m}$ with eigenvalue $-\frac{m b_{-}}{2}$.

An important feature of $\frac{i^{m}}{2} \Phi_{L, m, m, 0}(v, F)$ is that it is an eigenfunction of (minus) the operator $\Delta_{m}^{G}=\Delta_{m, 0}^{G}$. The proof is similar to the results appearing in Section 4.1 of [6]. However, as the regularization in [6] is different from ours, we give the proofs of all assertions, with an emphasis on the differences relative to [6].

Lemma 3.4. Let $f$ and $g$ be modular forms of weights $k$ and $k+2$ respectively, and representation $\rho_{L}$. The equality

$$
\int_{D_{w}}\left(y^{k+2}\left\langle R_{k} f, g\right\rangle+y^{k}\langle f, L g\rangle\right) y^{-s} d \mu=s \int_{D_{w}} y^{k+1}\langle f, g\rangle y^{-s} d \mu-\int_{\mathbb{R} / \mathbb{Z}+i w} w^{k}\langle f, g\rangle w^{-s} d x
$$

holds for any $w>1$ and $s \in \mathbb{C}$.

Proof. We follow the proof of Lemma 4.2 of [6] (with some sign differences). The first term on the right hand side arises from the difference between $y^{-s} d \omega$ and $d\left(y^{-s} \omega\right)$, for $\omega$ being the $S L_{2}(\mathbb{Z})$-invariant 1 -form $y^{k}\langle f, \bar{g}\rangle d \bar{\tau}$ appearing in [6]. Note that while $y^{-s} \omega$ is 
not $M p_{2}(\mathbb{Z})$-invariant in general, the fact that $y^{-s}$ is $T$-invariant and its restriction to the curve $|\tau|=1$ is also $S$-invariant (since $\Im(S \tau)=\frac{y}{|\tau|^{2}}$ ) allows us to apply the argument also in this case. This proves the lemma.

Using Lemma 3.4 twice yields the following analog of Lemma 4.3 of [6]:

Lemma 3.5. Let $f$ and $g$ be two modular forms of weight $k$ and representation $\rho_{L}$, and let $w>1$ and $s \in \mathbb{C}$ be given. The difference

$$
\int_{D_{w}} y^{k}\left\langle\Delta_{k} f, g\right\rangle y^{-s} d \mu-\int_{D_{w}} y^{k}\left\langle f, \Delta_{k} g\right\rangle y^{-s} d \mu
$$

equals

$$
s \int_{D_{w}} y^{k-1}(\langle L f, g\rangle-\langle f, L g\rangle) y^{-s} d \mu-\int_{\mathbb{R} / \mathbb{Z}+i w} w^{k-2}(\langle L f, g\rangle-\langle f, L g\rangle) w^{-s} d x .
$$

We are interested in the case where $g(\tau)=y^{\frac{b_{-}}{2}+m_{-}} \Theta_{L}\left(\tau, v, p_{v}\right)$ (so that $k=\frac{b_{+}}{2}+m_{+}-$ $\left.\frac{b_{-}}{2}-m_{-}\right)$. The line integrals at the limit $w \rightarrow \infty$ are dealt with in the following

Lemma 3.6. Let $L$ be an even lattice of signature $\left(b_{+}, b_{-}\right)$, let $v$ be an element of $G\left(L_{\mathbb{R}}\right)$ which does not belong to any $\lambda^{\perp}$ for $\lambda \in L^{*}$, and let $p$ be a polynomial on $L_{\mathbb{R}}$ which is homogenous of degree $\left(m_{+}, m_{-}\right)$with respect to $v$. Let $F$ be a modular form of weight $\frac{b_{+}}{2}+$ $m_{+}-\frac{b_{-}}{2}-m_{-}$and representation $\rho_{L}$, and write $F$ as in Eq. (9). Assume that for every $\gamma$ and $n$ we have $c_{\gamma, n}(y)=o\left(e^{\varepsilon y}\right)$ as $y \rightarrow \infty$ for every $\varepsilon>0$. In the case where $m_{+}+m_{-}$ is even and the constant $\Delta_{v}^{j}(p)$ for $j=\frac{m_{+}+m_{-}}{2}$ does not vanish, we assume further that $c_{0,0}(y)=o\left(y^{T}\right)$ as $y \rightarrow \infty$ for some $T$. Then

$$
\lim _{w \rightarrow \infty} \int_{\mathbb{R} / \mathbb{Z}} w^{\frac{b_{+}}{2}+m_{+}-2-s}\left\langle F(x+i w), \Theta_{L}\left(x+i w, v, p_{v}\right)\right\rangle_{\rho_{L}} d x=0
$$

for large enough $\mathfrak{\Re}$.

Proof. For fixed $w$, the integral equals some power of $w$ times the constant term of the Fourier expansion of $\left\langle F, \Theta_{L}\right\rangle$ at $y=w$. Hence we are considering the limit of the expression

$$
w^{\frac{b_{+}}{2}+m_{+}-2-s} \sum_{\lambda \in L^{*}} e^{-\Delta_{v} / 8 \pi w}(\bar{p})(\lambda) c_{\lambda, \frac{\lambda^{2}}{2}}(w) e^{-2 \pi w \lambda_{v_{+}}^{2}}
$$

as $w \rightarrow \infty$. As $v \notin \lambda^{\perp}$ for any $\lambda \in L^{*}$, we have $\lambda_{v_{+}}^{2}>0$ for any non-zero $\lambda \in L^{*}$, so that $e^{-2 \pi w \lambda_{v+}^{2}}$ eliminates the sub-exponential growth of $c_{\lambda, \frac{\lambda^{2}}{2}}(w)$. The contribution of the term with $\lambda=0$ may be non-zero only if $m_{+}+m_{-}$is even and the constant $\Delta_{v}^{j}(p)$ with $j=\frac{m_{+}+m_{-}}{2}$ does not vanish. In this case we consider some constant multiple of $c_{0,0}(w) w^{\frac{b_{+}}{2}+m_{+}-2-j-s}$, which tends to 0 for $\Re s \gg 0$ because of the polynomial growth of $c_{0,0}$. This proves the lemma.

Using Lemmas 3.5 and 3.6 we generalize Lemma 4.4 of [6] as follows. 
Lemma 3.7. Let $L, v, p$, and $F$ be as in Lemma 3.6. Assume that the regularized theta lift of F from Theorem 3.1 is well-defined. If $e^{-\Delta_{v} / 8 \pi y}(q)(\lambda=0)$ vanishes for either $q=p, q=$ $\Delta_{v_{+}} p$, or $q=\lambda_{v_{-}}^{2} p$, then the theta lift of $\Delta_{k} F(\tau)$, with the weight $k$ being $\frac{b_{+}}{2}+m_{+}-\frac{b_{-}}{2}+m_{-}$, gives the same result as the regularized integral

$$
\int_{X(1)}\left\langle F(\tau), \Delta_{k} y^{\frac{b_{+}}{2}+m_{+}} \Theta_{L}\left(\tau, \nu, p_{\nu}\right)\right\rangle_{\rho_{L}} \frac{d x d y}{y^{2}} .
$$

Proof. Fix $w>1$ and $s \in \mathbb{C}$. By Lemma 3.5, the difference between the corresponding integrals on $D_{w}$ is the sum of a line integral at $y=w$ and a certain integral over $D_{w}$ multiplied by $s$. The weight lowering operator $L$ preserves the properties of $F$ needed for Lemma 3.6, and by Eq. (6b) the image of $y^{\frac{b_{-}}{2}+m_{-}} \Theta_{L}\left(\tau, v, p_{v}\right)$ under $L$ is the sum of two theta functions with polynomials. Thus, for large enough $\Re s$, Lemma 3.6 implies that the line integral vanishes as $w \rightarrow \infty$. For the other integral, we may change the integral by a finite number without affecting the constant term in the Laurent expansion at $s=0$ because of the factor $s$. We thus replace the domain $D_{w}$ by the rectangle $x \in \mathbb{R} / \mathbb{Z}$ and $1 \leq$ $y \leq w$. Eq. (6b) reduces the expression we consider to a linear combination of integrals of the form

$$
s \int_{1}^{w} \int_{\mathbb{R} / \mathbb{Z}} y^{\alpha-s}\left\langle G(\tau), \Theta_{L}\left(\tau, v, q_{v}\right)\right\rangle d x d y
$$

for $(G, q)$ being $(L F, p),\left(F, \Delta_{v_{+}} p\right)$, and $\left(F, \lambda_{v_{+}}^{2} p\right)$, each with the corresponding power $\alpha$. Expanding $\left\langle G, \Theta_{L}\right\rangle$ and integrating over $x$ we obtain $s$ times the integral of a function of the form appearing in Eq. (14). Our assumption on $v$ implies that the integral over $[1, \infty)$ of every term with $\lambda \neq 0$ is finite for every $s$, so that the factor $s$ eliminates it at $s=0$. The difference between the theta lift and the integral asserted in the lemma is thus the constant term at $s=0$ of a linear combination of expressions of the form

$$
s \Delta_{v}^{j}(\bar{q}) \int_{1}^{\infty} y^{\alpha-j-s} c_{0,0}(y) d y
$$

for the appropriate $j$. Since we assume that the coefficient $\Delta_{v}^{j}(\bar{q})$ in Eq. (15) vanishes for the three possible polynomials $q$, the lemma follows.

In general the expression in Eq. (15) does not vanish. For example, if $F$ is weakly holomorphic then for large enough $\Re$ s Eq. (15) becomes a linear combination of expressions of the form $\frac{s}{\beta-s}$. Such a combination may have a non-zero constant term at $s=0$ in case a term with $\beta=0$ appears.

The conditions required by Lemma 3.6 and Lemma 3.7 are satisfied by a large variety of modular forms: Almost weakly holomorphic modular forms, various eigenforms of $\Delta_{k}$, and the functions from Definition 1.8 in Section 1.3 of [6] to name a few. These lemmas are thus applicable in many settings.

Returning to the theta lift $\Phi_{L, m, m, 0}(\nu, F)$ for $F=\delta_{1-\frac{b_{-}}{2}-m}^{m} f$ and $f$ weakly holomorphic, we obtain

Corollary 3.8. For $m>0$, or for $m=0$ (with $F=f$ ) under the condition $c_{0,0}=0$, the action of the operator $\Delta_{m}^{G}$ multiplies $\Phi_{L, m, m, 0}(v, F)$ by $2 m b_{-}$. 
Proof. The operator $\Delta_{m}^{G}=\widetilde{\Delta}_{m, 0}^{G}$ acts on the conjugated theta function $\overline{\Theta_{L, m, m, 0}}$ which shows up in the definition of $\Phi_{L, m, m, 0}$. Hence its action is the same as the action of the conjugated operator $\widetilde{\Delta}_{0, m}^{G}=\Delta_{0, m}^{G}+2 m b_{-}$on the theta function $\Theta_{L, m, m, 0}$ itself (after conjugating). But with $r=s=m$ and $t=0$, the combination $\Delta_{0, m}^{G}+2 m b_{-}$coincides with the operator appearing on the right hand side of Proposition 2.5. It follows that $\Delta_{m}^{G} \Phi_{L, m, m, 0}(v, F)$ is 4 times the integral from Lemma 3.7. Since for $m>0$ the polynomial $e^{-\Delta_{v} / 8 \pi y}\left(\bar{P}_{m, m, 0}\right)$ vanishes at $\lambda=0$ and for $m=0$ we assume $c_{0,0}=0$, the expression in Eq. (15) vanishes in both cases. Hence Lemma 3.7 implies that $\Delta_{m}^{G} \Phi_{L, m, m, 0}(v, F)$ coincides with $4 \Phi_{L, m, m, 0}\left(v, \Delta_{k} F\right)$. As $\Delta_{k}$ multiplies $F$ by $\frac{m b_{-}}{2}$, the corollary follows.

\subsection{The theta lift of $F=\delta_{1-\frac{b_{-}}{2}-m}^{m} f$}

We begin by evaluating $p_{v, h_{+}, h_{-}}$for $p_{v}=\frac{(-i)^{m}}{2} P_{m, m, 0}$. Only elements with $h_{-}=0$ appear (since $m_{-}=0$ ), and as $z_{v_{+}}=\frac{X_{v, V}}{Y^{2}}$ the binomial decomposition

$$
\frac{(-i)^{m}}{2} \cdot \frac{\left(\lambda, X_{v, V}+i Y_{\nu, V}\right)^{m}}{\left(Y^{2}\right)^{m}}=\sum_{h_{+}}\left(\begin{array}{c}
m \\
h_{+}
\end{array}\right)\left(\frac{\left(\lambda, X_{v, V}\right)}{Y^{2}}\right)^{h_{+}} \frac{i^{m-h_{+}}}{2 i^{m}} \cdot \frac{\left(\lambda, Y_{v, V}\right)^{m-h_{+}}}{\left(Y^{2}\right)^{m-h_{+}}}
$$

implies

$$
p_{v, h_{+}, 0}(\eta)=\frac{(-i)^{h_{+}}}{2}\left(\begin{array}{c}
m \\
h_{+}
\end{array}\right) \frac{(\eta, Y)^{m-h_{+}}}{\left(Y^{2}\right)^{m-h_{+}}} .
$$

This polynomial is homogenous of degree $\left(m-h_{+}, 0\right)$ with respect to the element $w \in$ $G\left(K_{\mathbb{R}}\right)$ in which $w_{+}$is spanned by $Y$ (or by the normalized generator $\frac{Y}{|Y|}$ ). This illustrates the reason for using the notation $p_{v, h_{+}, 0}$ rather than $p_{w, h_{+}, 0}$ : The latter notation allows dependence only on $w$, i.e., on $\frac{Y}{|Y|}$, while Eq. (16) displays dependence on $Y$ itself (hence on $v$ ). On the other hand, we can write $p_{v, 0,0}(\eta)$ as $\frac{1}{2|Y|^{m}}\left(\eta, w_{1}\right)^{m}$ with $w_{1}=\frac{Y}{|Y|}$ (as in Theorem 14.3 of [2]), so that the term with $\Phi_{K}$ in Theorem 7.1 of [2] can be evaluated using the polynomial $\tilde{p}(\eta)=\left(\eta, w_{1}\right)^{m}$. Since for this polynomial Theorem 10.3 of [2] remains valid, we deduce that $\Phi_{K}\left(v, F, p_{v, 0,0}\right)$ is a polynomial in $\frac{Y}{|Y|}$ divided by $|Y|^{m}$.

We can now state the properties of the theta lift $\Phi_{L, m, m, 0}(v, F)$.

Theorem 3.9. For $F=\delta_{1-\frac{b_{-}}{2}-m}^{m}$ and $f$ a weakly holomorphic modular form of weight $1-\frac{b_{-}}{2}-m$, the theta lift $\frac{i^{m}}{2} \Phi_{L, m, m, 0}(\nu, F)$ is a function of $Z \in K_{\mathbb{R}}+i C$ whose singularity along $\lambda^{\perp}$ for negative norm $\lambda$ is given by

$$
\begin{aligned}
& \frac{1}{2} \sum_{\alpha \lambda \in L^{*}} c_{\alpha \lambda, \frac{\alpha^{2} \lambda^{2}}{2}} \frac{(i \alpha)^{m}}{(2 \pi)^{m}}\left[\prod_{r=0}^{m-1}\left(r+\frac{b_{-}}{2}\right) \cdot \frac{\left(\lambda, \overline{Z_{v, V}}\right)^{m}}{2^{m}\left(Y^{2}\right)^{m}} \cdot\left(-\ln \frac{\left|\left(\lambda, Z_{v, V}\right)\right|^{2}}{Y^{2}}\right)+\right. \\
& \left.+\sum_{k=0}^{m-1} \frac{m !}{2^{k} k !}\left(\frac{\lambda^{2}}{2}\right)^{m-k} \cdot \prod_{r=0}^{k-1}\left(r+\frac{b_{-}}{2}\right) \cdot \frac{\left(\lambda, \overline{Z_{v, V}}\right)^{k}}{\left(\lambda, Z_{v, V}\right)^{m-k}\left(Y^{2}\right)^{k}} \cdot \frac{1}{m-k}\right] .
\end{aligned}
$$

This function is annihilated by $\Delta_{m}^{G}-2 m b_{-}$outside its singularities. Its Fourier expansion at the primitive norm 0 vector $z$ of $L$ (if it exists) decomposes, in a Weyl chamber $W$ containing $z$ in its closure, as

$$
\frac{\sqrt{2} \varphi(Y)}{\pi^{m-1}|Y|^{m-1}}+\sum_{k=0}^{m} \sum_{C=0}^{k} \sum_{\rho \in K^{*}} \frac{A_{k, C, \rho}}{\pi^{k+C}} \frac{(\rho, Y)^{k-C}}{\left(Y^{2}\right)^{k}} \times \begin{cases}\mathbf{e}((\rho, Z)) & (\rho, W)>0 \\ \mathbf{e}((\rho, \bar{Z})) & (\rho, W)<0 .\end{cases}
$$


Here $\varphi(Y)$ is a polynomial of degree not exceeding $m+1$ in $\frac{Y}{|Y|}$, plus some constant divided by $|Y|^{m+1}$. The constants $A_{k, C, \rho}$ involve rational numbers, roots of unity of some finite order, and the Fourier coefficients off.

Proof. The fact that $\Delta_{m}^{G} \Phi_{L, m, m, 0}=2 m b_{-} \Phi_{L, m, m, 0}$ is the content of Corollary 3.8. The singularities can be read off Theorem 3.2, with $b_{+}=2, m_{+}=m, k \leq m$, and $j=0$ since $p_{v}$ is harmonic. The condition $\beta \in-\mathbb{N}$ holds only for $k=m$, and the term with $\ln \left(\alpha^{2}\right)$ multiplies a smooth function. Substituting the coefficients $c_{\gamma, n, k}$ from Eq. (13) thus yields the asserted singularity near $\lambda^{\perp}$. The rest of the proof follows the proof of Theorem 14.3 of [2]. We assume that $L$ contains a primitive norm 0 vector (otherwise the assertion about Fourier expansions is vacuous), and that $v$ (or $w$ ) is not on any wall between two Weyl chambers. Since the polynomial $p_{v}$ is of homogeneity degree $(m, 0)$, we can take $h_{-}=$ $h=0$ in Theorem 7.1 of [2]. Thus, $\frac{i^{m}}{2} \Phi_{L, m, m, 0}(v, F)$ is the sum of $\frac{|Y|}{\sqrt{2}} \Phi_{K}\left(w, F_{K}, p_{v, 0,0}\right)$, a term coming from the element $0 \in K^{*}$, and the expression

$$
\begin{aligned}
& \sqrt{2}|Y| \sum_{h_{+}, j, k, n} \frac{n^{h_{+}}}{(2 i)^{h_{+}} j !(-8 \pi)^{j}} \sum_{0 \neq \eta \in K^{*}} \Delta_{w}^{j}\left(\bar{p}_{v, h_{+}, 0}\right)(\eta) \sum_{\gamma} \mathbf{e}(n[(\eta, X)+(\gamma, \zeta)]) \times \\
& \times c_{\gamma, \frac{\eta^{2}}{2}, k} \cdot 2\left(\frac{n Y^{2}}{2|(\eta, Y)|}\right)^{m-h_{+}-j-k-\frac{1}{2}} K_{m-h_{+}-j-k-\frac{1}{2}}(2 \pi n|(\eta, Y)|) .
\end{aligned}
$$

Here $\gamma$ is the $L^{*} / L$-image of an element of $L^{*}$ whose restriction to $z^{\perp} \subseteq L$ is the pullback of $\eta: K \rightarrow \mathbb{Z}$ under the projection $z^{\perp} \rightarrow K$. The derivation employs Lemma 7.2 of [2], together with the fact that $b_{+}=2, z_{v_{+}}^{2}=\frac{1}{Y^{2}}, \mu=X$, and $\eta_{w_{+}}^{2}=\frac{(\eta, Y)^{2}}{Y^{2}}$.

Next, applying $\Delta_{w_{+}}$(which differentiates twice with respect to the pairing with $\frac{Y}{|Y|}$ ) to the complex conjugate of $p_{v, h_{+}, 0}$ from Eq. (16) gives

$$
\Delta_{w}^{j}\left(\overline{p_{v, h_{+}, 0}}\right)(\eta)=\frac{i^{h_{+}}}{2} \cdot \frac{m !}{h_{+} !\left(m-h_{+}-2 j\right) !} \cdot \frac{(\eta, Y)^{m-h_{+}-2 j}}{\left(Y^{2}\right)^{m-h_{+}-j}} .
$$

Furthermore, we quote from the proof of Theorem 14.3 of [2] the formula

$$
K_{v+\frac{1}{2}}(t)=\sqrt{\frac{\pi}{2 t}} e^{-t} \sum_{r=0}^{v} \frac{(v+r) !}{r !(v-r) !} \frac{1}{(2 t)^{r}}
$$

for the $K$-Bessel function of half-integral index (here $v \in \mathbb{N}$, including 0 ). Since inverting the index leaves the $K$-Bessel function invariant, the same expansion holds for $K_{-v-\frac{1}{2}}(t)$. Substituting, and collecting the total powers of $2, \pi, n,(\eta, Y),|(\eta, Y)|$, and $Y^{2}$ we find that this expression reduces to

$$
\begin{aligned}
& \sum_{h_{+}, j, k, n} \sum_{\eta, \gamma, r} \frac{m !(v+r) ! c}{h_{+} \frac{\eta^{2}}{2}, k}(-1)^{j} \\
& \times|(\eta, Y)|^{k-j-r} \operatorname{sgn}(\eta, Y)^{m-h_{+}}\left(Y^{2}\right)^{-k} \mathbf{e}(n[(\eta, X)+i|(\eta, Y)|+(\gamma, \zeta)]) .
\end{aligned}
$$

Here $v$ stands for $m-h_{+}-j-k-1$ when this integer is non-negative and for $j+k+h_{+}-m$ otherwise. We observe that $j$ and $r$ appear in exponents only through their $\operatorname{sum} C=j+r$, and we wish to show that only terms with $C \leq k$ appear. Considering the terms in which $v=j+k+h_{+}-m$, we recall that $2 j$ is bounded by $m-h_{+}$. Hence we have the inequality 
$j+h_{+}-m \leq-j$, which implies $r \leq v \leq k-j$ hence $C=j+r \leq k$. For the other terms we fix $C$ and write $r=C-j$, so that the only part depending on $j$ is

$$
\frac{1}{C !} \sum_{j}(-1)^{j}\left(\begin{array}{l}
C \\
j
\end{array}\right) \frac{\left(m-h_{+}+C-k-1-2 j\right) !}{\left(m-h_{+}-2 j\right) !} .
$$

For $C \geq k+1$ this sum equals

$$
\frac{(C-k-1) !}{C !} \sum_{j}(-1)^{j}\left(\begin{array}{l}
C \\
j
\end{array}\right)\left(\begin{array}{c}
m-h_{+}+C-k-1-2 j \\
C-k-1
\end{array}\right),
$$

hence vanishes by Lemma 3.3 since the rightmost binomial coefficient is a polynomial of degree $C-k-1<C$ in $j$. Thus, only terms with $C \leq k$ survive, which implies that $|(\eta, Y)|^{k-C}$ is continuous for every $\eta$.

Now, by Eq. (13), $c_{\gamma, \frac{\eta^{2}}{2}, k}$ is some rational multiple of $c_{\gamma, \frac{\eta^{2}}{2}} \frac{\left(\eta^{2}\right)^{m-k}}{\pi^{k}}$. Thus, given $k, C, n, \eta$, and $\gamma$ (with $C \leq k$ and the usual relation between $\gamma$ and $\eta$ ), the corresponding terms in Eq. (18) take the form

$$
a_{k, C, \varepsilon} \frac{n^{m-k-C-1}\left(\eta^{2}\right)^{m-k}}{\pi^{k+C}} c_{\gamma, \frac{\eta^{2}}{2}} \frac{(\eta, Y)^{k-C}}{\left(Y^{2}\right)^{k}} \mathbf{e}(n[(\eta, X)+i|(\eta, Y)|+(\gamma, \zeta)]),
$$

where $\varepsilon=\operatorname{sgn}(\eta, Y)$ and the coefficients $a_{k, C, \varepsilon}$ are rational numbers. Borcherds [2] shows that $a_{0,0, \varepsilon}$ equals $2^{m}$ for $\varepsilon=+1$ and 0 for $\varepsilon=-1$. Now, the power of $n$ lies in $\mathbb{Q}$, and raising $\mathbf{e}((\gamma, \zeta))$ to a power which equals the level of $L$ gives 1 . The remaining part of the exponent is $\mathbf{e}(n(\eta, Z))$ if $(\eta, Y)>0$ and $\mathbf{e}(n(\eta, \bar{Z}))$ if $(\eta, Y)<0$. We may substitute $n \eta=\rho$, and the total coefficient of $\frac{(\rho, Y)^{k-C}}{\pi^{k+C}\left(Y^{2}\right)^{k}}$ times $\mathbf{e}((\rho, Z))$ or $\mathbf{e}((\rho, \bar{Z}))$ becomes

$$
A_{k, C, \rho}=a_{k, C, \varepsilon}\left(\rho^{2}\right)^{m-k} \sum_{n>0, \frac{\rho}{n} \in L^{*}} \sum_{\left.\gamma\right|_{z^{\perp}}=\frac{\rho}{n}} \frac{c^{c} \frac{\rho^{2}}{2 n^{2}}}{n^{m+1}} \mathbf{e}(n(\gamma, \zeta)) .
$$

This coefficient clearly has the asserted properties. As the dependence on $X=\mu$ comes only from $\mathbf{e}((\eta, \mu))$, the term with $\Phi_{K}$ and the term with $\eta=0$ both go into $\varphi(Y)$.

Now, the term involving $\Phi_{K}$ was discussed above (note the factor $\frac{1}{||^{\mid m}}$ from $p_{v, 0,0}$ but also the coefficient $\sqrt{2}|Y|$ from Theorem 7.1 of [2]). The reason for the coefficient $\frac{\sqrt{2}}{\pi^{m-1}}$ is explained below. The polynomial from Theorem 10.3 of [2] has degree at most $m+1$ since $m_{+}=k_{\text {max }}=m$ and $m_{-}=0$. For the term with $\eta=0$, fix $h_{+}$and $j$. As $\Delta_{w}^{j}\left(\bar{p}_{v, h_{+}, 0}\right)(\eta)$ does not vanish for $\eta=0$ only if $2 j=m-h_{+}$, we fix only $j$ and write $h_{+}=m-2 j$. Recall that for $\eta=0$ the values which $\gamma \in L^{*} / L$ attains are the images of $\frac{\delta z}{N}$ for $\delta \in \mathbb{Z} / N \mathbb{Z}$ (where $N$ is defined by $(L, z)=N \mathbb{Z}$ ). Combining this with Eq. (17) and Lemma 7.3 of [2] thus yields the expression

$$
\begin{aligned}
& \sqrt{2}|Y| \sum_{j, k, n} \frac{n^{m-2 j}}{(2 i)^{m-2 j} j !(-8 \pi)^{j}} \cdot \frac{i^{m-2 j}}{2} \cdot \frac{m !}{(m-2 j) !\left(Y^{2}\right)^{j}} \sum_{\delta \in \mathbb{Z} / N \mathbb{Z}} \mathbf{e}\left(\frac{\delta n}{N}\right) \times \\
& \times c_{\frac{\delta z}{N}, 0, k} \cdot\left(\frac{\pi n^{2} Y^{2}}{2}\right)^{j-k-\frac{1}{2}-s} \Gamma\left(s+\frac{1}{2}+k-j\right) .
\end{aligned}
$$

Collecting powers gives

$$
\begin{aligned}
& \sum_{k, \delta} c_{\frac{\delta z}{N}, 0, k} \frac{2^{k-m}}{\left(Y^{2}\right)^{k} \pi^{k+\frac{1}{2}}} \sum_{j} \frac{(-1)^{j} m !}{4^{j} j !(m-2 j) !} \Gamma\left(s+\frac{1}{2}+k-j\right)\left(\frac{2}{\pi Y^{2}}\right)^{s} \times \\
& \times \sum_{n} \mathbf{e}\left(\frac{\delta n}{N}\right) n^{m-2 k-1-2 s} .
\end{aligned}
$$


The sum over $n$ equals $N^{m-2 k-1-2 s} \sum_{\varepsilon=1}^{N} \mathbf{e}\left(\frac{\delta \varepsilon}{N}\right) \zeta\left(1+2 k-m+2 s, \frac{\varepsilon}{N}\right)$, involving values of the Hurwitz zeta function. Now, Eq. (13) implies that $c_{\frac{\delta z}{N}, 0, k}$ equals $\prod_{r=0}^{k-1}\left(r+\frac{b_{-}}{2}\right) \cdot \frac{c^{c} \frac{\delta z}{N}, 0}{(4 \pi)^{m}}$ for $k=m$ and vanishes otherwise. Since the Hurwitz zeta function is holomorphic at $m+1$, the constant term of this expression at $s=0$ is obtained by substitution. The fact that $\Gamma\left(\frac{1}{2}+k-j\right) \in \mathbb{Q} \cdot \sqrt{\pi}$ renders the expression from Eq. (20)

$$
\frac{\tilde{a}}{\left(Y^{2}\right)^{m} \pi^{2 m}} \sum_{\delta \in \mathbb{Z} / N \mathbb{Z}} c_{\frac{\delta z}{N}, 0} \sum_{\varepsilon=1}^{N} \mathbf{e}\left(\frac{\delta \varepsilon}{N}\right) \zeta\left(m+1, \frac{\varepsilon}{N}\right)
$$

with some $\tilde{a} \in \mathbb{Q}$. Since this is a constant times $\frac{1}{\left(Y^{2}\right)^{m}}$, adding it to the term with $\Phi_{K}$ shows that $\varphi(Y)$ has the desired form. This proves the theorem.

A remark about the evaluation of the constant term $P_{2}$ in Theorem 14.3 of [2] is in order here. In the case of weakly holomorphic $F$ considered there, only $k=0$ appears. Evaluating the Hurwitz zeta function thus obtained and using the symmetry and duplication formulae for the gamma function shows that the true value of $P_{2}$ is half the value given in the proof of Theorem 14.3 of [2]. The constant appearing in the assertion of that theorem is, on the other hand, correct. One should thus be careful when evaluating $P_{2}$ as the limit of the expression for $\eta \neq 0$ (indeed, with $(\eta, W)<0$ one gets 0$)$. In any case, the proof of Theorem 3.9 shows how to evaluate this term directly.

Note that the coefficients of the polynomial part of $\varphi(Y)$ involve the lattice $K$, some roots of unity, and the Fourier coefficients of the modular form $f$ (this is the reason for the coefficient $\frac{\sqrt{2}}{\pi^{m-1}}$ ). Hence for "algebraic" $f$ these coefficients are algebraic. On the other hand, evaluating the constant coming from Eq. (20) for $\eta=0$ is very hard. For example, for $N=1$ we must take even $m$, yielding the values of the Riemann zeta function at odd positive integers, whose properties (not to mention a finite formula) are not yet known.

We remark that Theorem 3.9 extends to the case $m=0$, if $c_{0,0}=0$. In general, $\Delta_{0}^{G} \frac{1}{2} \Phi_{L, 0,0,0}(\nu, F)$ is a non-zero constant multiple of $c_{0,0}$. Indeed, in this case one obtains the usual theta lift with $p=1$ from Theorem 13.3 of [2] (up to a factor of 2), $\ln |\Psi|^{2}$ is harmonic for meromorphic $\Psi$, and $\Delta_{0}^{G}=8 \Omega$ sends $\ln |Y|$ to a non-zero constant by Equation (4.3) of [6]. We mention that Theorem 3.9, as well as Theorems 4.6 and 4.7 of [6], provide answers to Problem 16.6 of [2] in some (interesting) special cases.

We remark that $\frac{i^{m}}{2} \Phi_{L, m, m, 0}(v, F)$ can be written in terms of the weakly holomorphic modular form $f$ itself, namely as $\frac{(-i)^{m}}{2^{m+1}} \Phi_{L}\left(v, f, p_{v}\right)$ with the polynomial $p_{v}(\lambda)=$ $P_{m, m, 0}(\lambda, Z)\left(\lambda_{v_{-}}^{2}\right)^{m}$ (recall that $P_{m, m, 0}$ is $\Delta_{v_{+}}$-harmonic). This is a consequence of Eq. (6b), using an argument similar to Lemmas 3.4, 3.6, and 3.7. This is useful in case one wishes to apply the embedding trick from Section 8 of [2]. Indeed, the lifted modular form is then divided by the classical holomorphic cusp form $\Delta$ of weight 12 (not to be confused with the various Laplacians, whose notation also involves the symbol $\Delta$ ). Now, if $f$ is weakly holomorphic then so is $\frac{f}{\Delta}$, while if $F$ is an eigenfunction of $\Delta_{k}$ then $\frac{F}{\Delta}$ does not necessarily share this property.

\subsection{The case $b_{-}=1$}

In the case $b_{-}=1$ we may consider our lattices as embedded in the vector space $V=$ $M_{2}(\mathbb{R})_{0}$ of traceless $2 \times 2$ real matrices. This space has signature $(2,1)$ with $(A, B)=$ $\operatorname{Tr}(A B)$ and $A^{2}=-2 \operatorname{det} A$. Take $z=\left(\begin{array}{cc}0 & 1 / \beta \\ 0 & 0\end{array}\right)$ for some $\beta>0$ and $\zeta=\left(\begin{array}{cc}0 & -h / 2 \beta \\ \beta & 0\end{array}\right)$ (where 
$\left.h=\zeta^{2}\right)$. Then $K_{\mathbb{R}} \cong\{z, \zeta\}^{\perp}$ is the space of traceless diagonal matrices. We send $x \in \mathbb{R}$ to $\left({ }^{\beta x}{ }_{-\beta x}\right) \in K_{\mathbb{R}}$ with the norm $2 \beta^{2} x^{2}$, and take $C$ to be the cone of positive reals. Thus $G(V) \cong K_{\mathbb{R}}+i C$ is identified with $\mathcal{H}$. Explicitly, given $\tau \in \mathcal{H}$, the vector $Z_{v, V}$ is $\beta M_{\tau}$ and $v_{-}=\mathbb{R} J_{\tau}$ using the matrices defined in the beginning of Section 1. Automorphic forms are modular forms of twice the weight, as Eq. (2) shows. The group $S L_{2}(\mathbb{R})$ acts on $V$ by conjugation, yielding an isomorphism $P S L_{2}(\mathbb{R}) \cong S O^{+}(V)$. We remark that right multiplication by $S$ (and rescaling the bilinear form) gives the model in which the vector space consists of the symmetric $2 \times 2$ matrices from Example 5.1 of [3]. In order for $z$ to be a primitive norm 0 vector in the lattice $L$ considered there we must take $\beta=\sqrt{N}$.

Consider now $\Gamma^{+}=\Gamma \cap S O^{+}\left(L_{\mathbb{R}}\right)$ (in the case $b_{-}=1$ ) as a Fuchsian subgroup of $P S L_{2}(\mathbb{R}) . \frac{i^{m}}{2} \Phi_{L, m, m, 0}(v, F)$ is then a modular form of weight $2 m$ on $\mathcal{H}$ with respect to $\Gamma^{+}$, which has eigenvalue $-2 m$ with respect to $\Delta_{m}^{G}=\Delta_{2 m}$. A negative norm vector $\lambda \in L^{*}$ is a multiple of $J_{\sigma}$ for some $\sigma=s+i t \in \mathcal{H}$, and then $\lambda^{\perp}=\{\sigma\}$. By a slight abuse of notation, we replace the variable $v \in G(V)$ of $\Phi$ by $\tau=x+i y$ once more (as we consider only $\Phi$ and not $\Theta$ here, this should lead to no confusion). The description of $\frac{i^{m}}{2} \delta_{2 m} \Phi_{L, m, m, 0}(v, F)$ is given in

Theorem 3.10. The function $\frac{i^{m}}{2} \delta_{2 m} \Phi_{L, m, m, 0}(v, F)$ is a meromorphic modular form of weight $2 m+2$ with respect to $\Gamma^{+}$. Its poles are at points $\sigma \in \mathcal{H}$ for which a real multiple of $J_{\sigma}$ lies in (the isomorphic copy of) $L^{*}$. The principal part at such $\sigma$ is

$$
\frac{i}{(4 \pi)^{m+1}} \sum_{\alpha J_{\sigma} \in L^{*}} c_{\alpha J_{\sigma},-\alpha^{2}} \frac{\alpha^{m}}{\beta^{m}} \cdot \frac{m !(2 i t)^{m+1}}{(\tau-\sigma)^{m+1}(\tau-\bar{\sigma})^{m+1}} .
$$

In case $\Gamma^{+}$has cusps, the Fourier expansion at such a cusp is

$$
\sum_{r>0} \frac{r^{m}}{\beta^{2 m}} \sum_{d \mid r} d^{m+1} \sum_{\left.\gamma\right|_{z^{\perp}}=\frac{d}{2 \beta^{2}}} c_{\gamma, \frac{d^{2}}{4 \beta^{2}}} \mathbf{e}\left(\frac{r}{d}(\gamma, \zeta)\right) q^{r},
$$

plus some constant if $m=0$.

Proof. We first observe that applying $\delta_{2 m}$ to a modular form of weight $2 m$ having eigenvalue $-2 m$ yields a meromorphic modular form of weight $2 m+2$. The singularities of $\frac{i^{m}}{2} \delta_{2 m} \Phi_{L, m, m, 0}(v, F)$ are the $\delta_{2 m}$-images of the singularities of $\frac{i^{m}}{2} \Phi_{L, m, m, 0}(v, F)$ given in Theorem 3.9. The constant $\prod_{r=0}^{k-1}\left(r+\frac{b_{-}}{2}\right)$ is $\frac{(2 k) !}{4^{k} k !}$, for $\lambda=J_{\sigma}$ we have $\lambda^{2}=-2$, and $Y^{2}$ equals $2 \beta^{2} y^{2}$. The pairing of $Z_{\nu, V}=\beta M_{\tau}$ and of $\overline{Z_{\nu, V}}=\beta \overline{M_{\tau}}$ with $\lambda=J_{\sigma}$ give $-\frac{\beta}{t}(\tau-\sigma)(\tau-\bar{\sigma})$ and its complex conjugate respectively. Hence the singularity of $\frac{i^{m}}{2} \Phi_{L, m, m, 0}(v, F)$ at $\sigma$ is

$$
\begin{aligned}
& \frac{1}{2} \sum_{\alpha J_{\sigma} \in L^{*}} c_{\alpha J_{\sigma},-\alpha^{2}} \frac{(i \alpha)^{m}}{(2 \pi)^{m}}\left[\frac{(2 m) !}{4^{m} m !} \cdot \frac{[-(\bar{\tau}-\sigma)(\bar{\tau}-\bar{\sigma})]^{m}}{\left(4 y^{2}\right)^{m} \beta^{m} t^{m}} \cdot\left(-\ln \frac{|(\tau-\sigma)|^{2}|(\tau-\bar{\sigma})|^{2}}{2 y^{2}}\right)+\right. \\
& \left.\quad+\sum_{k=0}^{m-1} \frac{m !(-1)^{m-k}}{2^{k} k !} \cdot \frac{(2 k) !}{4^{k} k !} \frac{[-(\bar{\tau}-\sigma)(\bar{\tau}-\bar{\sigma})]^{k} t^{m-2 k}}{[-(\tau-\sigma)(\tau-\bar{\sigma})]^{m-k} \beta^{m}\left(2 y^{2}\right)^{k}} \cdot \frac{1}{m-k}\right] .
\end{aligned}
$$

We write this expression as

$$
\frac{m !}{2} \sum_{\alpha J_{\sigma} \in L^{*}} c_{\alpha J_{\sigma},-\alpha^{2}}\left(\frac{i \alpha t}{2 \pi \beta}\right)^{m} \sum_{k=0}^{m} \frac{(2 k) !(-1)^{k}}{(k !)^{2}(4 y t)^{2 k}} \cdot(\bar{\tau}-\sigma)^{k}(\bar{\tau}-\bar{\sigma})^{k} g_{k}(\tau),
$$


with $g_{k}(\tau)$ being $\frac{1}{(m-k)(\tau-\sigma)^{m-k}(\tau-\bar{\sigma})^{m-k}}$ for $0 \leq k<m$ and $-\ln \frac{|(\tau-\sigma)|^{2}|(\tau-\bar{\sigma})|^{2}}{2 y^{2}}$ for $k=m$. Applying $\delta_{2 m}$ reduces to letting $\delta_{2 m-2 k}$ operate on $g_{k}$ for each $k$. Hence the singularity of $\frac{i^{m}}{2} \delta_{2 m} \Phi_{L, m, m, 0}(\nu, F)$ at $\sigma$ is

$$
\begin{gathered}
\frac{m ! i}{4 \pi} \sum_{\alpha J_{\sigma} \in L^{*}} c_{\alpha J_{\sigma},-\alpha^{2}}\left(\frac{i \alpha t}{2 \pi \beta}\right)^{m} \sum_{k=0}^{m} \frac{(2 k) !(-1)^{k}}{(k !)^{2}(4 y t)^{2 k}}(\bar{\tau}-\sigma)^{k}(\bar{\tau}-\bar{\sigma})^{k} \times \\
\times \frac{(\tau-\bar{\sigma})+(\tau-\sigma)+\frac{2(\tau-\sigma)(\tau-\bar{\sigma})}{-2 i y}}{(\tau-\sigma)^{m+1-k}(\tau-\bar{\sigma})^{m+1-k}} .
\end{gathered}
$$

For ease of presentation we omit, until the last step, the coefficient in front of the sum over $k$. Expand the $k$ th powers of $\bar{\tau}-\sigma=(\tau-\sigma)-2 i y$ and $\bar{\tau}-\bar{\sigma}=(\tau-\bar{\sigma})-2 i y$, and write $\frac{(-1)^{k}}{(4 y t)^{2 k}}$ as $\frac{1}{(2 t)^{2 k}(-2 i y)^{2 k}}$. This gives

$$
\begin{aligned}
\sum_{k=0}^{m} & \frac{(2 k) !}{(2 t)^{2 k}} \sum_{a, b} \frac{1}{a !(k-a) ! b !(k-b) !(-2 i y)^{a+b}}\left[\frac{1}{(\tau-\sigma)^{m+1-k-a}(\tau-\bar{\sigma})^{m-k-b}}+\right. \\
& \left.+\frac{1}{(\tau-\sigma)^{m-k-a}(\tau-\bar{\sigma})^{m+1-k-b}}+\frac{\frac{2}{-2 i y}}{(\tau-\sigma)^{m-k-a}(\tau-\bar{\sigma})^{m-k-b}}\right] .
\end{aligned}
$$

Now fix $c$ and $l$, and collect the terms involving $\frac{1}{(\tau-\sigma)^{m-k-l+1}(-2 i y)}$. We thus take $a=l$ and $b=c-l$ in the first summand within the square brackets, $a=l-1$ and $b=c-l+1$ in the second summand, and $a=l-1$ and $b=c-l$ in the third summand. This gives

$$
\begin{aligned}
\sum_{k, c, l} & \frac{(2 k) !(2 t)^{-2 k}}{(\tau-\sigma)^{m-k-l+1}(\tau-\bar{\sigma})^{m-k-c+l}(-2 i y)^{c}}\left[\frac{1}{l !(k-l) !(c-l) !(k+l-c) !}+\right. \\
& \left.\quad+\frac{1}{(l-1) !(k+1-l) !(c-l+1) !(k+l-1-c) !}+\frac{2}{(l-1) !(k+1-l) !(c-l) !(k+l-c) !}\right] .
\end{aligned}
$$

The sum of the combinatorial expressions reduces to $\frac{(k+1)(c+1)}{l !(k+1-l) !(c-l+1) !(k+l-c) !}$, so that we can write the latter expression as

$$
\sum_{c} \frac{(c+1)(-2 i y)^{-c}}{(\tau-\sigma)^{m+1}(\tau-\bar{\sigma})^{m+1-c}} \sum_{k, l} \frac{(2 k) !(k+1)(\tau-\sigma)^{k+l}(\tau-\bar{\sigma})^{k+1-l}}{(2 t)^{2 k} l !(k+1-l) !(c-l+1) !(k+l-c) !} .
$$

Expand the power of $\tau-\bar{\sigma}=\tau-\sigma+2 i t$, and write $(2 t)^{2 k}=(-1)^{k}(2 i t)^{2 k}$. We thus obtain for every $c$ a sum of the form

$$
\sum_{k, l, h} \frac{(-1)^{k}(2 k) !(k+1)(\tau-\sigma)^{k+l+h}}{(2 i t)^{k+l+h-1} l !(c-l+1) !(k+l-c) ! h !(k+1-l-h) !} .
$$

The index change $k=s-l-h$ yields

$$
\sum_{c, s} \frac{(-1)^{s}(c+1)(-2 i y)^{-c}(2 i t)^{1-s}}{(\tau-\sigma)^{m+1-s}(\tau-\bar{\sigma})^{m+1-c}} \sum_{l, h} \frac{(-1)^{l+h}(2 s-2 l-2 h) !(s-l-h+1)}{l !(c-l+1) !(s-h-c) ! h !(s+1-2 l-2 h) !} .
$$

For every $c$ and $s$ now write $r=h+l$, and the corresponding term becomes

$$
\sum_{r} \frac{(-1)^{r}(2 s-2 r) !(s-r+1)}{(s+1-2 r) !(c+1) !(s-c) !} \sum_{l}\left(\begin{array}{c}
c+1 \\
l
\end{array}\right)\left(\begin{array}{c}
s-c \\
r-l
\end{array}\right) .
$$

A well-known combinatorial identity shows that the sum over $l$ is simply $\left(\begin{array}{c}s+1 \\ r\end{array}\right)$. By writing $(s+1-r)\left(\begin{array}{c}s+1 \\ r\end{array}\right)$ as $(s+1)\left(\begin{array}{l}s \\ r\end{array}\right)$ and $\frac{c+1}{(c+1) !(s-c) !}$ as $\frac{1}{s !}\left(\begin{array}{l}s \\ c\end{array}\right)$, the expression in question reduces to

$$
\sum_{c, s} \frac{(-1)^{s}(s+1)(-2 i y)^{-c}(2 i t)^{1-s}}{(\tau-\sigma)^{m+1-s}(\tau-\bar{\sigma})^{m+1-c} s !}\left(\begin{array}{l}
s \\
c
\end{array}\right) \sum_{r}(-1)^{r}\left(\begin{array}{l}
s \\
r
\end{array}\right) \frac{(2 s-2 r) !}{(s+1-2 r) !}
$$


As $\frac{(2 s-2 r) !}{(s+1-2 r) !}=\prod_{i=s+2}^{2 s}(i-2 r)$ is a polynomial of degree $s-1<s$ for all $s>0$, Lemma 3.3 shows that the sum over $r$ vanishes unless $s=0$. Thus only the term $\frac{2 i t}{(\tau-\sigma)^{m+1}(\tau-\bar{\sigma})^{m+1}}$, corresponding to $s=c=0$, survives. Multiplying by the factor we omitted above now gives the asserted singularity at $\sigma$.

Assume that $\Gamma^{+}$has a cusp, corresponding to a primitive norm 0 vector $z \in L$ which is normalized as above. Theorem 3.9 shows that for $b_{-}=1$, the expansion of $\frac{i^{m}}{2} \Phi_{L, m, m, 0}(\nu, F)$ at the cusp is the sum of an almost holomorphic function of depth $\leq 2 m$, the complex conjugate of such a function, and an expression of the form $\frac{B}{y^{m-1}}+\frac{D}{y^{2 m}}$ for some constants $B$ and $D$. Note that the condition on the sign of $(\rho, W)$ implies a sub-exponential growth at the cusp. Moreover, the eigenvalue $-2 m$ implies that $B=0$ (unless $m=0$ ) and that the conjugate almost holomorphic part combines with $\frac{D}{y^{2 m}}$ to a give $\frac{h(\tau)}{y^{2 m}}$ with $h$ anti-holomorphic. $\delta_{2 m}$ thus annihilates this part. Now, if the almost holomorphic part is $\sum_{r} \frac{\psi_{r}(\tau)}{y^{r}}$ with meromorphic functions $\psi_{r}$ then the meromorphicity of $\frac{i^{m}}{2} \delta_{2 m} \Phi_{L, m, m, 0}(v, F)$ shows that its expansion is just $\frac{\psi_{0}^{\prime}(\tau)}{2 \pi i}$. We normalize $z$ and $\beta$ such that $K^{*}=\frac{1}{2 \beta^{2}} \mathbb{Z} \subseteq \mathbb{R}$ and $(\rho, W)>0$ is equivalent, for $\rho=\frac{r}{2 \beta^{2}}$, to $r>0$. Then the part with $(\rho, W)>0$ in the expansion of $\frac{i^{m}}{2} \Phi_{L, m, m, 0}(v, F)$ in Theorem 3.9 is $\sum_{k, C} A_{k, C, \rho} \frac{r^{k-C}}{\left(2 \beta^{2}\right)^{k}(\pi y)^{k+C}} \mathbf{e}(r \tau)$. Hence $\psi_{0}(\tau)=\sum_{r>0} A_{0,0, \frac{r}{2 \beta^{2}}} q^{r}$, so that $\frac{\psi_{0}^{\prime}(\tau)}{2 \pi i}=\sum_{r>0} r A_{0,0, \frac{r}{2 \beta^{2}}} q^{r}$. We substitute $A_{k, C, \rho}$ from Eq. (19), the value $a_{0,0,+1}=2^{m}$, and the norm $\frac{s^{2}}{2 \beta^{2}}$ of $\frac{s}{2 \beta^{2}} \in K^{*}$, and make the index change $d=\frac{r}{n}$. This yields the asserted Fourier expansion for $\frac{i^{m}}{2} \delta_{2 m} \Phi_{L, m, m, 0}(v, F)$ at the cusp, and completes the proof of the theorem.

Note that the Fourier coefficients in Theorem 3.10 are based, up to rational numbers and the global power of $\beta$, on roots of unity of bounded order and the Fourier coefficients of the weight $\frac{1}{2}-m$ weakly holomorphic modular form $f$.

As an example for the map $f \mapsto \frac{i^{m}}{2} \delta_{2 m} \Phi_{L, m, m, 0}(v, F)$, fix $N \in \mathbb{N}$, and take $L$ to be the lattice spanned by $z=\left(\begin{array}{cc}0 & 1 / \sqrt{N} \\ 0 & 0\end{array}\right), \zeta=\left(\begin{array}{cc}0 & 0 \\ \sqrt{N} & 0\end{array}\right)$, and the vector $\left(\begin{array}{c}\sqrt{N} \\ -\sqrt{N}\end{array}\right)$. In this case $\beta=\sqrt{N}, L^{*} / L=K^{*} / K$ is cyclic of order $2 N, \rho_{L}=\rho_{K},(z, L)=\mathbb{Z}$ and $\zeta \in L$, and $\Gamma^{+}=\Gamma_{0}(N)$ acts by conjugation. Hence Theorem 3.10 yields a singular Shimura-type correspondence, as stated in the following

Corollary 3.11. Let $f(\tau)=\sum_{\gamma \in \mathbb{Z} / 2 N \mathbb{Z}} \sum_{n \in \mathbb{Q}} c_{\gamma, n} q^{n} e_{\gamma}$ be a weakly holomorphic modular form of weight $\frac{1}{2}-m$ and representation $\rho_{K}$ for some $m \in \mathbb{N}$. The q-series

$$
\sum_{r>0} \frac{r^{m}}{N^{m}}\left(\sum_{d \mid r} d^{m+1} c_{d, \frac{d^{2}}{4 N}}\right) q^{r}
$$

defines a meromorphic modular form of weight $2 m+2$ with respect to $\Gamma_{0}(N)$, which has poles of order $m+1$ at some points in $\mathcal{H}$ which are quadratic over $\mathbb{Q}$.

In the case $N=1$ in Corollary 3.11 the modular forms of $\frac{1}{2}-m$ and representation $\rho_{L}$ correspond to weakly holomorphic modular forms of the same weight with respect to $\Gamma_{0}(4)$ which lie in the corresponding Kohnen plus-space (see Chapter 5 of [12] or Proposition 1 of [18], which easily generalize to the weakly holomorphic case). They 
exist only if $m$ is even (since $L^{*} / L$ has exponent 2), yielding the statement given in the corresponding Theorem in the Introduction.

The subsequent work [29] investigates the behavior of $\frac{i^{m}}{2} \Phi_{L, m, m, 0}$ under weight changing operators for automorphic forms on Grassmannians (defined in that reference) in higher dimensions. As meromorphic images are constructed in that reference, it should be possible to obtain a Gross-Kohnen-Zagier type theorem for higher-codimensional Heegner cycles in universal families over higher-dimensional varieties along the lines of the proof of the main result of this paper. On the other hand, [30] investigates the modular forms from Theorem 3.10 further, and relates them to the results of [4] as well as to other modular objects.

\section{Relations between $\mathrm{CM}$ cycles}

Let $B$ and $i$ be as in Section 1 , and let $L$ be the lattice $\tilde{\Lambda} \subseteq B_{0}$ from part (ii) of Corollary 1.5. The arithmetic applications of the theory of theta lifts are based on

Proposition 4.1. The following assertions hold:

(i) The map $\frac{i}{\sqrt{\text { disc(I) }}}$ identifies $L_{\mathbb{R}}$ with $M_{2}(\mathbb{R})_{0}$ as quadratic spaces.

(ii) A point $\tau \in \mathcal{H}$ is a $C M$ point for $B$ and i if and only if it is of the form $\lambda^{\perp}$ for some $\lambda$ from $L^{*}$.

Proof. Part (i) follows from part (ii) of Theorem 1.4. For part (ii) use the description for $G\left(L_{\mathbb{R}}\right)$ preceding Theorem 3.10 and apply Proposition 1.13 . This proves the proposition.

The observation from Proposition 4.1 relates the special points $\lambda^{\perp}$ on $\mathcal{H}$ as a Grassmannian to the CM points on modular and Shimura curves. This shows that the automorphic forms of [2] have divisors with arithmetic meaning, and as we shall see below, similar assertion holds for the singularities of our automorphic forms.

\section{1 $m$-Divisors, Heegner $m$-Divisors, and their classes}

We now define the objects for which we prove the main result. Let $L$ be an even lattice of signature $\left(2, b_{-}\right)$, and let $G\left(L_{\mathbb{R}}\right)$ be its positive Grassmannian.

Definition 4.2. An m-divisor on $G\left(L_{\mathbb{R}}\right)$ is a locally finite sum of elements in $S y m^{m} L_{\mathbb{C}} \otimes$ $\operatorname{Div}\left(G\left(L_{\mathbb{R}}\right)\right)$, namely of expressions of the sort

$$
\left(\prod_{i=1}^{m} \lambda_{i}\right) \otimes Y
$$

where $\lambda_{i} \in L_{\mathbb{C}}$ and $Y \subseteq G\left(L_{\mathbb{R}}\right)$ is an irreducible divisor. Recalling that $O\left(L_{\mathbb{R}}\right)$ acts on both $G\left(L_{\mathbb{R}}\right)$ and Sym ${ }^{m} L_{\mathbb{C}}$, we define an m-divisor on the quotient $X(\Gamma)=\Gamma \backslash G\left(L_{\mathbb{R}}\right)$ to be an $m$-divisor on $G\left(L_{\mathbb{R}}\right)$ which is invariant under the action of the discrete subgroup $\Gamma$ of $O\left(L_{\mathbb{R}}\right)$. An m-divisor on $G\left(L_{\mathbb{R}}\right)$ or on $X(\Gamma)$ is of totally negative type if it satisfies the following condition: For every term of the form appearing in Eq. (21), each $\lambda_{i}$ lies in (the complexification of) the space $v_{-}$corresponding to every point $v \in Y$. This condition is 
well-defined, and is equivalent to every term being of the form $a_{\lambda} \lambda^{m} \otimes \lambda^{\perp}$ for some negative norm vector $\lambda \in M_{\mathbb{R}}$, with $a_{\lambda} \in \mathbb{C}$.

So $m$-divisors are divisors with coefficients in local systems. Lemma 1.9 and part (ii) of Theorem 1.4 show that the local system from Definition 4.2 becomes $\mathrm{Sym}^{m} V_{2}$ when $b_{-}=1$.

We need to introduce another property of automorphic forms on $G\left(L_{\mathbb{R}}\right)$. Let $\Phi$ be an automorphic form of weight $m$ on $G\left(L_{\mathbb{R}}\right) \cong K_{\mathbb{R}}+i C$ which is an eigenform of $\Delta_{m}^{G}$ with eigenvalue $-2 m b_{-}$, and assume first that the lattice $L$ contains a primitive norm zero vector $z$. The expansion of $\Phi$ around $z$ in a Weyl chamber $W$ containing $z$ in its closure is of the form

$$
\widetilde{\varphi}(Y)+\sum_{k=0}^{m} \sum_{C=0}^{k} \sum_{\rho \in K^{*}} \frac{A_{k, C, \rho}}{\pi^{k+C}} \frac{(\rho, Y)^{k-C}}{\left(Y^{2}\right)^{k}} \times \begin{cases}\mathbf{e}((\rho, Z)) & (\rho, W)>0 \\ \mathbf{e}((\rho, \bar{Z})) & (\rho, W)<0\end{cases}
$$

(as in Theorem 3.9). We call $\Phi$ algebraic if the coefficients $A_{k, C, \rho}$ are algebraic over $\mathbb{Q}$. If $L$ contains no norm zero vectors, then as in Section 8 of [2], we can embed $L$ in two different lattices $M$ and $N$ of signature $\left(2, b_{-}+24\right)$, giving embeddings of $G\left(L_{\mathbb{R}}\right)$ into the larger Grassmannians $G\left(M_{\mathbb{R}}\right)$ and $G\left(N_{\mathbb{R}}\right)$. Assume that we can present $\Phi$ as the difference of weight $m$ automorphic forms on $G\left(M_{\mathbb{R}}\right)$ and $G\left(N_{\mathbb{R}}\right)$, which are eigenforms with eigenvalue $-2 m\left(b_{-}+24\right)$ of the corresponding Laplacians, minus their singularities. We call $\Phi$ algebraic if it is obtained in this way from algebraic automorphic forms on the larger Grassmannians. We note again that one has to apply the argument carefully in this casesee the last remark following Theorem 3.9 above. The $q$-expansion principle shows that in the case where $b_{-}=1$ and $\Gamma$ has cusps, the $\delta_{2 m}$-images of an algebraic automorphic form of automorphic weight $m$ is an algebraic meromorphic modular form of $2 m+2$. This assertion is likely to extend also to the case where $\Gamma$ has no cusps.

Let $\Gamma$ be a discrete subgroup of $O^{+}\left(L_{\mathbb{R}}\right)$ (we will take the intersection of the discriminant kernel with $\mathrm{SO}^{+}(L)$ in our applications). In general we make the following

Definition 4.3. The m-divisor of totally negative type on $X(\Gamma)$, specified as $\sum_{\lambda \in L_{\mathbb{R}}, \lambda^{2}<0} a_{\lambda} \lambda^{m} \otimes \lambda^{\perp}$ (a locally finite, $\Gamma$-invariant sum) is called strongly principal if there exists an algebraic automorphic form of weight $m$ on $G\left(L_{\mathbb{R}}\right)$ whose singularities lie on the divisors $\lambda^{\perp}$, and along each such $\lambda^{\perp}$ the singularity is

$$
\begin{aligned}
& \frac{a_{\lambda}}{2}\left(\frac{i}{2 \pi}\right)^{m}\left[\prod_{r=0}^{m-1}\left(r+\frac{b_{-}}{2}\right) \cdot \frac{\left(\lambda, \overline{Z_{v, V}}\right)^{m}}{2^{m}\left(Y^{2}\right)^{m}} \cdot\left(-\ln \frac{\left|\left(\lambda, Z_{v, V}\right)\right|^{2}}{Y^{2}}\right)+\right. \\
& \left.\quad+\sum_{k=0}^{m-1} \frac{m !}{2^{k} k !}\left(\frac{\lambda^{2}}{2}\right)^{m-k} \cdot \prod_{r=0}^{k-1}\left(r+\frac{b_{-}}{2}\right) \cdot \frac{\left(\lambda, \overline{Z_{v, V}}\right)^{k}}{\left(\lambda, Z_{v, V}\right)^{m-k}\left(Y^{2}\right)^{k}} \cdot \frac{1}{m-k}\right] .
\end{aligned}
$$

If $b_{-}=1$ we adopt the alternative

Definition 4.4. We call the m-divisor of totally negative type on $X(\Gamma)$ defined by $\sum_{\lambda \in L_{\mathbb{R}}, \lambda^{2}<0} a_{\lambda} \lambda^{m} \otimes \lambda^{\perp}$ (again a locally finite, $\Gamma$-invariant sum) principal if there exists an 
algebraic modular form of weight $2 m+2$ on $G\left(L_{\mathbb{R}}\right) \cong \mathcal{H}$, whose poles are at the points $\lambda^{\perp}$, and at each point $\lambda^{\perp}=\sigma$ the singularity is

$$
\frac{i}{(4 \pi)^{m+1}} \sum_{\alpha} a_{\alpha J_{\sigma}} \alpha^{m} \frac{m !(2 i t)^{m+1}}{(\tau-\sigma)^{m+1}(\tau-\bar{\sigma})^{m+1}} .
$$

We remark that while the (principal) divisor of a rational function depends also on the zeros of the function, our (strongly) principal $m$-divisors involve only the singularities of the function. Indeed, when comparing the case $m=0$ here with [3], the theta lift $\Phi$ from [2] (considered in Definition 4.3) is (roughly) the logarithm of the absolute value of the Borcherds product $\Psi$, and for $b_{-}=1$ Definition 4.4 considers the singularities of $\delta_{0} \Phi=\frac{1}{2 \pi i} \frac{\partial \Phi}{\partial \tau} \sim \frac{\Psi^{\prime}}{\Psi}$, the logarithmic derivative of $\Psi$ (see also the remarks at the end of this Section). The algebraicity constraint corresponds to the fact that the Fourier coefficients of negative indices of the weakly holomorphic modular form appearing in Theorem 13.3 of [2] are required to be integral.

A subtle point in Definitions 4.3 and 4.4 is dealt with in the following

Lemma 4.5. If the two terms $a_{\lambda} \lambda^{m} \otimes \lambda^{\perp}$ and $a_{\mu} \mu^{m} \otimes \mu^{\perp}$ coincide then so are the corresponding singularities in Definitions 4.3 and 4.4 .

Proof. The assumption of the lemma is equivalent to $\lambda=x \mu$ and $a_{\mu}=x^{m} a_{\lambda}$ for some real $x \neq 0$. As $\left(\frac{\lambda^{2}}{2}\right)^{m-k} \cdot \frac{\left(\lambda, \overline{Z_{v, V}}\right)^{k}}{\left(\lambda, Z_{v, V}\right)^{m-k}}$ equals $x^{m}\left(\frac{\mu^{2}}{2}\right)^{m-k} \cdot \frac{\left(\mu, \overline{Z_{v, V}}\right)^{k}}{\left(\mu, Z_{v, V}\right)^{m-k}}$ for every $0 \leq k \leq m$ in this case (and the difference in the logarithm gives a smooth function of $Z$ ), the assertion holds for Definition 4.3. For Definition 4.4, with $b_{-}=1$, we have $\lambda=\alpha J_{\sigma}$ for some $\alpha \in \mathbb{R}$ and $\sigma \in \mathcal{H}$. Then $\mu=\frac{\alpha}{x} J_{\sigma}$, and the assertion follows from the equality $\alpha^{m}=x^{m}\left(\frac{\alpha}{x}\right)^{m}$. This proves the lemma.

Given $\gamma \in M^{*} / M$ and $n \in \mathbb{Q}$, let $y_{n, \gamma}^{(m)}=\sum_{\lambda \in L+\gamma, \lambda^{2}=-2 n} \lambda^{m} \otimes \lambda^{\perp}$. It is trivial if $n \not \equiv \gamma^{2} / 2(\bmod \mathbb{Z})$, and also if $2 \gamma=0$ in $L^{*} / L$ and $m$ is odd. In particular, if $L^{*} / L$ has exponent 2 (or 1) then $y_{n, \gamma}^{(m)}=0$ for all $n$ and $\gamma$ if $m$ is odd. The group Heeg $g^{(m)}(X(\Gamma))$ of Heegner $m$-divisors is the free Abelian group of $m$-divisors which is generated by the $m$-divisors $y_{n, \gamma}^{(m)}$. Let PrinHeeg $g_{s t}^{(m)}(X(\Gamma))$ be the subgroup of $\mathrm{Heeg}^{(m)}(X(\Gamma))$ consisting of those Heegner $m$-divisors which are strongly principal. If $b_{-}=1$ then we denote the group of principal Heegner $m$-divisors by $\operatorname{PrinHeeg}^{(m)}(X(\Gamma))$. The spaces $\mathrm{Heeg}_{s t}^{(m)} \mathrm{Cl}(X(\Gamma))$ and $H_{e e g}{ }^{(m)} \mathrm{Cl}(X(\Gamma))$ of (strong) Heegner m-divisor classes on $X(\Gamma)$ are the corresponding quotient groups. We denote the image of $y_{n, \gamma}^{(m)}$ in these quotients by $y_{n, \gamma}^{(m)}$ as well.

We can now state and prove the main result of this paper.

Theorem 4.6. The formal power series $\sum_{n, \gamma} y_{n, \gamma}^{(m)} q^{n} e_{\gamma}$ is a modular form of weight $1+\frac{b_{-}}{2}+m$ and representation $\rho_{L}^{*}$ with respect to $M p_{2}(\mathbb{Z})$, with coefficients in the finite-dimensional space $\mathrm{Heeg}_{s t}^{(m)} \mathrm{Cl}(X(\Gamma)) \otimes \mathbb{Q}$.

Proof. The proof follows [3]. Consider the space of weight $k$ modular forms with representation $\rho_{L}^{*}$ (as a finite-dimensional subspace of the space of power series), and the space of singular parts of weakly holomorphic modular forms of weight $2-k$ and representation $\rho_{L}$ (which is a subspace of finite codimension in the space of all singular parts). By 
Theorem 3.1 of [3], these two spaces are the full perpendicular spaces of one another for any half-integral $k$. Let $f$ be a weakly holomorphic modular form of weight $1-\frac{b_{-}}{2}-m$ and representation $\rho_{L}$ with respect to $M p_{2}(\mathbb{Z})$. Expand $f$ as in Eq. (9). Then Theorem 3.9 shows that the singularities of the theta lift $\frac{i^{m}}{2} \Phi_{L, m, m, 0}(v, F)$ of $F=\delta_{1-\frac{b_{-}}{2}-m} f$ are along the divisors $\lambda^{\perp}$ with $\lambda \in L^{*}$, where every $\lambda \in L^{*}$ contributes a singularity along $\lambda^{\perp}$ which takes the form appearing in Definition 4.3 with $a_{\lambda}=c_{\lambda, \frac{\lambda^{2}}{2}}$. If we assume that $f$ has algebraic Fourier coefficients, then it follows from the description of the coefficients $A_{k, C, \rho}$ that the automorphic form $\frac{i^{m}}{2} \Phi_{L, m, m, 0}(v, F)$ is algebraic. Assuming that the Fourier coefficients $c_{n, \gamma}$ with $n<0$ are integral, this implies that the $m$-divisor $\sum_{n>0, \gamma} c_{\gamma,-n} y_{n, \gamma}^{(m)}$ is strongly principal, hence it vanishes in $\operatorname{Heeg}_{s t}^{(m)} \mathrm{Cl}(X(\Gamma))$. Let $\xi^{(m)}$ be the map which takes a "singular part" $\sum_{n<0, \gamma} a_{\gamma, n} q^{n} e_{\gamma}$ to the element $\sum_{n>0, \gamma} a_{\gamma,-n} y_{n, \gamma}^{(m)}$ in the class group $\mathrm{Heeg}_{s t}^{(m)} \mathrm{Cl}(X(\Gamma))$. The map $\xi^{(m)}$ is surjective, and since the space of weakly holomorphic modular forms of any weight and representation $\rho_{L}$ has a basis consisting of modular forms with integral Fourier coefficients (see [19]), $\xi^{(m)}$ factors through the quotient of the space of singular parts by singular parts of weakly holomorphic modular forms. By Lemmas 4.2, 4.3, and 4.4 of [3] this quotient space is finite-dimensional. Hence $\operatorname{dim} \mathrm{Heeg}_{s t}^{(m)} \mathrm{Cl}(X(\Gamma)) \otimes \mathbb{Q}<\infty$ as well. Thus the Serre duality pairing extends to the case where the space of singular parts and the image of the pairing are tensored with $\mathrm{Heeg}_{s t}^{(m)} \mathrm{Cl}(X(\Gamma))$, and continues to be non-degenerate.

Now, for an algebraic modular form $f$ as above, the vanishing expression $\sum_{n>0, \gamma} c_{\gamma,-n} y_{n, \gamma}^{(m)}$ is obtained as the Serre duality pairing of the formal power series $\sum_{n, \gamma} y_{n, \gamma}^{(m)} q^{n} e_{\gamma}$ with the singular part of $f$. Hence this power series is perpendicular to all the singular parts of weakly holomorphic meromorphic modular form of weight $1-\frac{b_{-}}{2}-m$ and representation $\rho_{L}$ with respect to $M p_{2}(\mathbb{Z})$. By Lemma 4.3 of [3] and the result of [19], the formal power series in question lies in the space of (holomorphic) modular forms of weight $1+\frac{b_{-}}{2}+m$ and representation $\rho_{L}^{*}$ tensored with $\mathrm{Heeg}_{s t}^{(m)} \mathrm{Cl}\left(X_{\Gamma}\right) \otimes \mathbb{C}$. The fact that the Serre duality pairing and the spaces we consider are defined over $\mathbb{Q}$ implies the "algebraicity" of $\sum_{n, \gamma} y_{n, \gamma}^{(m)} q^{n} e_{\gamma}$ as a modular form with coefficients in $\mathrm{Heeg}^{(m)} \mathrm{Cl}\left(X_{\Gamma}\right) \otimes \mathbb{Q}$. This proves the theorem.

In the case $b_{-}=1$ we obtain an assertion like that of Theorem 4.6 but with coefficients in $\mathrm{Heeg}^{(m)} \mathrm{Cl}(X(\Gamma)) \otimes \mathbb{Q}$ (no subscript $s t$ ). This follows either by omitting this subscript and replacing Definition 4.3 by Definition 4.4 in the proof of Theorem 4.6, or using the fact that every strongly principal Heegner $m$-divisor is principal. Indeed, if $\Phi$ is an automorphic form which shows that some $m$-divisor is strongly principal, then $\delta_{2 m} \Phi$ reveals the principality of this $m$-divisor. Whether or not the notions of principality and strong principality are equivalent in the case $b_{-}=1$ remains an interesting question. We also remark that unlike the result of [3], here the "constant term" $y_{0,0}$ does not show up. Thus our expression is in fact a "cusp form".

Let us give an example of an automorphic form yielding the principality of a Heegner $m$-divisor. This also illustrates Corollary 3.11 above. Take $N=1$ in the example preceding Corollary 3.11, and let $m$ be even. Theorem 4.6 implies that the generating series of the Heegner $m$-divisors is a cusp form of weight $\frac{3}{2}+m$ and representation $\rho_{L}^{*}$. Equivalently, it lies in the Kohnen plus-space of cusp forms of weight $\frac{3}{2}+m$ on $\Gamma_{0}(4)$ (see [12] or [18]). This space is isomorphic to the space of cusp forms of weight $2 m+2$ on $S L_{2}$ (Z) by 
Theorem 1 of [18]. Hence this series may not vanish only if $m \geq 8$. We take $m=2$, and present the function yielding the principality $y_{\frac{3}{4}, 1}^{(2)}$. Define

$$
\theta(\tau)=\sum_{n \in \mathbb{Z}} q^{n^{2}} \quad \text { and } \quad H_{\frac{5}{2}}(\tau)=120 \sum_{n=0}^{\infty} H(2, n) q^{n}
$$

as in [18] (here $H(2, n)$ is the function introduced in [11], and $H_{\frac{5}{2}}$ is normalized to attain 1 at $\infty$ and to have integer coefficients). Let $E_{l}$ be the classical (normalized) Eisenstein series of even weight $l \geq 4$ on $S L_{2}(\mathbb{Z})$, and let $\Delta$ be the classical cusp form of weight 12 . The weight $-\frac{3}{2}$ weakly holomorphic modular form $f$ with representation $\rho_{L}$ having the appropriate principal part corresponds to the weakly holomorphic modular form

$$
\begin{aligned}
& g(\tau)=\frac{E_{10}(4 \tau) \theta(\tau)-E_{8}(4 \tau) H_{\frac{5}{2}}(\tau)}{\Delta(4 \tau)}= \\
& =q^{-3}-56+384 q-15024 q^{4}+39933 q^{5}-523584 q^{8}+1129856 q^{9}+O\left(q^{12}\right)
\end{aligned}
$$

in the Kohnen plus-space of weight $-\frac{3}{2}$. The lift $\frac{i^{2}}{2} \delta_{4} \Phi_{L, 2,2,0}(v, F)$ of $F=\delta_{-\frac{3}{2}}^{2} f$ is given by the Fourier expansion

$$
384 q-479232 q^{2}+274558464 q^{3}-118219210752 q^{4}+43867326009600 q^{5}+O\left(q^{6}\right)
$$

around $\infty$. It is a modular form of weight 6 with respect to $S L_{2}(\mathbb{Z})$, which has a pole of the form $\frac{18 \sqrt{3} /(4 \pi)^{3}}{(\tau-\sigma)^{3}(\tau-\bar{\sigma})^{3}}$ at $\sigma$ the 3 rd root of unity in $\mathcal{H}$ (recall that $\beta$ and the Fourier coefficient are 1 and $\alpha=2 t=\sqrt{3})$. These properties determine $\frac{i^{2}}{2} \delta_{4} \Phi_{L, 2,2,0}(v, F)$ as $\frac{384 E_{6} \Delta}{E_{4}^{3}}$, a fact which can also be verified directly by an explicit evaluation of the Fourier coefficients.

\subsection{Algebaricity and relations to other works}

We now explain why the condition of algebraicity of the automorphic form is required in the definition of principal $m$-divisors (both in Definition 4.3 and in Definition 4.4). The eigenvalue of the function $\frac{i^{m}}{2} \Phi_{L, m, m, 0}(v, F)$ from Theorem 3.9 under the action of $\Delta_{m}^{G}$ is a consequence of the fact that $F=\delta_{1-\frac{b_{-}-m}{2}}^{m} f$ has a specific eigenvalue under the weight $1-\frac{b_{-}}{2}+m$ Laplacian on $\mathcal{H}$. Replacing the weakly holomorphic modular form $f$ by a harmonic weak Maaß form would yield $F=\delta_{1-\frac{b_{-}}{2}-m}^{m} f$ with the same eigenvalue. Hence $\frac{i^{m}}{2} \Phi_{L, m, m, 0}(\nu, F)$ would have the same properties. For further details on harmonic weak Maaß forms we refer the reader to Section 3 of [7].

Now, every harmonic weak Maaß form $f$ of weight $l$ and representation $\rho_{L}$ with respect to $M p_{2}(\mathbb{Z})$ decomposes as the sum of a holomorphic part (which yields a similar expression in the theta lift) and a non-holomorphic part. The non-holomorphic part consists of "constant terms" multiplying a power of $y$ and expressions which are based on incomplete gamma functions. Hence the part of $F=\delta_{l}^{m} f$ arising from the non-holomorphic part of $f$ is again a power of $y$, plus the complex conjugate of an almost holomorphic function, plus similar incomplete gamma functions. For $l<1-m$ the map $\delta_{l}^{m} f$ is invertible (its inverse is a constant multiple of the $m$ th power of the weight lowering operator $L)$. Thus every modular form $F$ of weight $l+2 m$ and representation $\rho_{L}$ with respect to $M p_{2}(\mathbb{Z})$ having eigenvalue $m(l+m-1)$ is $\delta_{l}^{m} f$ for some harmonic weak Maaß form $f$. Take $l=1-\frac{b_{-}}{2}-m$, and consider first the case where the operator $\xi_{l}$ of [7] takes $f$ to a weakly holomorphic modular form which is not holomorphic at the cusp. An argument similar to Section 6 of [2] (or Theorem 3.2) shows that the corresponding theta lift 
$\frac{i^{m}}{2} \Phi_{L, m, m, 0}(v, F)$ from Theorem 3.9 has, apart from the usual singularities, also singularities along the sub-Grassmannians of the form $\left\{v \in G\left(L_{\mathbb{R}}\right) \mid \lambda \in v_{+}\right\}$for $\lambda \in L^{*}$ with positive norm (these are $\lambda^{\perp}$ on $G\left(L_{\mathbb{R}}(-1)\right) \cong G\left(L_{\mathbb{R}}\right)$ ). In the case $b_{-}=1$ these lifts may be related to (non-harmonic) locally Maaß forms as defined in [5], and their $\delta_{2 m}$-images are probably examples of the locally harmonic Maaß forms considered in that reference. In any case, lifts of such forms do not yield additional principal Heegner $m$-divisors.

Consider now the case where $\xi_{1-\frac{b_{-}-m}{2}} f$ is holomorphic at the cusp. The singularity then arises only from the holomorphic part of $f$. Now, if $L$ contains a norm zero vector $z$ then the Fourier expansion of $\frac{i^{m}}{2} \Phi_{L, m, m, 0}(v, F)$ contains, apart from the expression from Theorem 3.9, terms involving Bessel $K$-functions evaluated at $2 \pi n|Y| \cdot\left|\eta_{w_{-}}\right|=$ $2 \pi n \sqrt{(\eta, Y)^{2}-Y^{2} \eta^{2}}>0$ (the strict positivity follows from the condition on $\xi_{1-\frac{b_{-}-m}{2}} f$, since only elements with $\eta^{2} \leq 0$ contribute in the non-holomorphic part of $f$ ). Hence one may distinguish, for $b_{-}>1$, the theta lifts arising from weakly holomorphic modular forms from those in which $\xi_{1-\frac{b_{-}-m}{2}} f \neq 0$ by the fact that the former do not include terms of the form $\left.\mathbf{e}\left((\rho, X)+i \sqrt{(\rho, Y)^{2}-Y^{2} \rho^{2}}\right)\right)$. Note that for $b_{-}=2$, with $(\rho, Z)=\rho_{1} \sigma+\rho_{2} \tau$, the latter expression is either $\mathbf{e}\left(\rho_{1} \sigma+\rho_{2} \bar{\tau}\right)$ or $\mathbf{e}\left(\rho_{1} \bar{\sigma}+\rho_{2} \tau\right)$. These expressions may thus be related to the class map defined in Section 5 of [6] in terms of the operator $\partial \bar{\partial}$. On the other hand, in case $b_{-}=1$ the lattice $K$ is positive definite. Hence only the holomorphic part of $f$ contributes to the theta lift. As any principal part at $\infty$ can be obtained as the principal part of a harmonic weak Maaß form of every weight (see Proposition 3.11 of [7]), omitting the algebraicity condition in Definitions 4.3 and 4.4 would reduce the the (strong) Heegner $m$-divisor class group (for $b_{-}=1$ ) to 0 . Now, (algebraic) weakly holomorphic modular forms give rise to algebraic theta lifts, while for harmonic weak Maaß forms we pose the following

Conjecture 4.7. Let $f$ be a harmonic weak Maaß form of weight $\frac{1}{2}-m$ (with $m>0$ ) and representation $\rho_{L}$, and assume that $\xi_{\frac{1}{2}-m} f$ is holomorphic at $\infty$. Assume further that the principal part of $f$ involves only Fourier coefficients which are algebraic over $\mathbb{Q}$, or even integral. Then the condition $\xi_{\frac{1}{2}-m} f \neq 0$ implies the existence of some Fourier coefficient of the holomorphic part of $f$ which is transcendental over $\mathbb{Q}$.

Although we do not investigate the Fourier coefficients of harmonic weak Maaß forms in this paper, let us indicate what evidence towards Conjecture 4.7 does exist. First, we mention Corollary 1.4 of [8], as well as the conjecture preceding it in that reference. Indeed, in the weight $\frac{1}{2}$ case considered there one has certain theta series of weight $\frac{3}{2}$ which map by the Shimura correspondence to Eisenstein series of weight 2. Harmonic weak Maaß forms which map to these forms under the operator $\xi_{\frac{1}{2}}$ are known to have algebraic Fourier coefficients. Since in higher half-integral weights the Shimura correspondence takes cusp forms to cusp forms, we expect in Conjecture 4.7 only the weakly holomorphic modular forms to have algebraic coefficients. In any case, it will be interesting to find, under Conjecture 4.7, whether every relation between the Heegner $m$-divisors in $\mathrm{Heeg}_{s t}^{(m)} \mathrm{Cl}\left(X(\Gamma)\right.$ ) (or in $\mathrm{Heeg}^{(m)} \mathrm{Cl}(X(\Gamma))$ for $b_{-}=1$ ) arises from a weakly holomorphic modular form as we described.

Theorems 0.2.1 and 0.3.1 of [31] (with $k=m+1$ ) relate the images of $m$-dimensional $\mathrm{CM}$ cycles in the Kuga-Sato variety $W_{2 m}$ over modular curves in a certain vector space 
to modular forms of weight $2 m+2$. Such a modular form may be related to the modular form of weight $\frac{3}{2}+m$ obtained from our Theorem 4.6 by the Shimura-Shintani correspondence. This may also indicate, once a relation between our construction and the one in [31] is established, that Conjecture 4.7 may be true, since otherwise the power series from Theorem 4.6 must lie in a strict quotient Hecke module. Furthermore, [17] defines, for any $m$, a map on the Heegner divisors on $X_{0}(N)$ into some elliptic curve. This reference conjectures that the images of these divisors under this map correspond to the coefficients of a modular form of weight $2 m+2$. In some cases this map coincides with the Abel-Jacobi map on the CM cycles into a certain sub-torus of the intermediate Jacobian of the Kuga-Sato variety $W_{2 m}$. The paper [17] also supplies numerical evidence that this is true in some other cases as well.

Now, the results of Section 1 relate the Heegner $m$-divisors in the case $b_{-}=1$ to symmetric powers of normalized cohomology classes of Heegner cycles inside Kuga-Sato type varieties. In the case $m=0$ considered in [3], the automorphic form $\delta_{0} \frac{1}{2} \Phi_{L, 0,0,0}$ is roughly the logarithmic derivative of the Borcherds product $\Psi$. Hence it is (under some normalization, when $c_{0,0}=0$ ) a meromorphic modular form of weight 2 with only simple poles in CM points, and the residues in these poles are integral. It thus corresponds to a differential of the third kind in the description of [24] or Section 3 of [8]. Its algebraicity thus corresponds to the fact that its residue divisor vanishes in the Jacobian of the curve $X(\Gamma)$ (being the divisor of the rational function $\Psi$ )- see Theorem 1 of [24] or Theorem 3.2 of [8]. Returning to the case of general $m$, we attach the element $M_{\tau}^{m}$, or $\left(\begin{array}{l}\tau \\ 1\end{array}\right)^{2 m}$, of the local system $S_{y m}{ }^{m} L_{\mathbb{C}}$, to the meromorphic modular form $\delta_{2 m} \frac{i^{m}}{2} \Phi_{L, m, m, 0}$. We thus obtain a meromorphic modular form of weight 2 and representation $V_{2 m}$. This corresponds to a meromorphic differential form of degree $2 m+1$ on (the Shimura curve analog of) $W_{2 m}$. Pairing this modular form with $\lambda^{m} \otimes \lambda^{\perp}$ for $\lambda=\alpha J_{\sigma}$ gives the function $\delta_{2 m} \frac{(i \alpha)^{m}}{2 t^{m}} \Phi_{L, m, m, 0}(\tau)(\tau-\sigma)^{m}(\tau-\bar{\sigma})^{m}$ (up to normalization), whose pole at $\sigma$ is simple. Moreover, if we assume that $f$ has integral Fourier coefficients, then the residues are integral. In addition, it seems that the corresponding differential of the third kind is canonical in the following sense: The cohomology group $H^{2 m+1}\left(W_{2 m}\right)$ has a component $H^{1}\left(X, V_{2 m}\right)$ of Hodge weight $(2 m+1,0)$ and $(0,2 m+1)$ whose holomorphic part consists of $g\left(\begin{array}{l}\tau \\ 1\end{array}\right)^{2 m} d \tau$ for $g$ a cusp form of weight $2 m+2$ with respect to $\Gamma$. This is shown explicitly for $m=1$ in Section 6 of [1], and it is not hard to generalize to arbitrary $m$. The $H^{1}\left(X, V_{2 m}\right)$ part of the cohomology class which is Poincaré dual to a cycle $\mathcal{Z}$ of real dimension $2 m+1$ in $W_{2 m}$ equals $g(\tau)\left(\begin{array}{l}\tau \\ 1\end{array}\right)^{2 m} d \tau+\overline{g(\tau)}\left(\begin{array}{l}\bar{\tau} \\ 1\end{array}\right)^{2 m} d \bar{\tau}$ for some cusp form $g$. We should therefore have the equality

$$
\int_{\mathcal{Z}} \delta_{2 m} \frac{i^{m}}{2} \Phi_{L, m, m, 0}\left(\begin{array}{l}
\tau \\
1
\end{array}\right)^{2 m} d \tau=\int_{X(\Gamma)}(2 i y)^{2 m} \delta_{2 m} \frac{i^{m}}{2} \Phi_{L, m, m, 0} \overline{g(\tau)} d \tau d \bar{\tau}
$$

(up to the poles). As the latter form is exact like in Lemma 3.4, the integral reduces to the contribution from the poles, which also vanishes since $\bar{g}$ is smooth and the integral over a circle of radius $\varepsilon$ around each pole $\sigma$ gives an expression which vanishes as $\varepsilon \rightarrow 0$. This evaluation process is well-defined up to the location of the poles of $\delta_{2 m} \frac{i^{m}}{2} \Phi_{L, m, m, 0}$ with respect to the choice of the cycle. However, jumping over a pole $\sigma$ changes the value by a totally imaginary multiple of the residue of $\delta_{2 m} \frac{i^{m}}{2} \Phi_{L, m, m, 0}(\tau-\sigma)^{m}(\tau-\bar{\sigma})^{m}$, which is assumed to be integral. It may be interesting to compare this evaluation with the regularized integrals from [4]. In any case, if we could establish some generalization 
of Theorem 1 of [24] assuring us that the algebraicity of this differential form with local coefficients is equivalent to the vanishing of a certain expression inside some generalized Jacobian (up to torsion), then we would know that the images of our Heegner $m$-divisors in $\mathrm{Heeg}^{(m)} \mathrm{Cl}(X(\Gamma))$ are the same as their images in this Jacobian.

The map $\alpha$ of [17] also seems to be related to the $\left(H^{2 m+1,0}\right)^{*}$ part of the intermediate Jacobian of the Kuga-Sato variety $W_{2 m}$. For any CM point $\sigma$, consider the $m$ th symmetric power $z_{\sigma}^{m}$ of the normalized CM cycle corresponding to $\sigma$ in $A_{\sigma}$. In addition, consider the closure of the $(2 m+1)$-dimensional cycle $[\sigma, \infty) \times z_{\sigma}^{m}$ in the notation of Section 8 of [1] (in the modular case this is possible) plus some cycle bounding the fiber over $\infty$. This resembles, in the case $m=1$, the cycle considered in [23], where the latter part is the counterpart of the combination of the cycles $\Delta_{k}$ from [1]. Then the integral of some form $g(\tau)\left(d z_{1} \wedge d z_{2}\right)^{m} d \tau$ of type $(2 m+1,0)$ over this cycle decomposes as in the proof of Theorem 8.5 of [1]. The integral from $\sigma$ to $\infty$ is of the function $g(\tau)(\tau-\sigma)^{m}(\tau-\bar{\sigma})^{m}$ (up to some constant). Since $\sigma$ is a (modular) CM point on $\mathcal{H}$, this gives (under the correct normalization) the integral considered in [17]. The integral over the cycle in the fiber over $\infty$ probably gives some period of $g$, and when we focus on one newform $g$ (of weight $2 m+2$ ) this reduces to the image modulo the lattice in $\mathbb{C}$ appearing in [17]. It will be very interesting to investigate what relations can be established between the existence of the form $\delta_{2 m} \frac{i^{m}}{2} \Phi_{L, m, m, 0}$ and the values of these integrals.

Authors' information

The initial stage of this research has been carried out as part of my Ph.D. thesis work at the Hebrew University of Jerusalem, Israel. The final stage of this work was carried out at the Technical University of Darmstadt, Germany and supported by the Minerva Fellowship (Max-Planck-Gesellschaft).

Received: 22 April 2015 Accepted: 7 September 2015

Published online: 07 December 2015

References

1. Besser, A: CM cycles over Shimura curves. J. Algebraic Geometry. 4, 659-692 (1993)

2. Borcherds, RE: Automorphic forms with singularities on Grassmannians. Invent. Math. 132, 491-562 (1998)

3. Borcherds, RE: The Gross-Kohnen-Zagier Theorem in higher dimensions. Duke Math J. 97(2), 219-233 (1999). Correction: Duke Math J., vol 105 no. 1, 183-184 (2000)

4. Bringmann, K, Kane, B: Cycle integrals of meromorphic modular forms and CM-values of automorphic forms, submitted for publication. a.i. http://arxiv.org/abs/1409.0793

5. Bringmann, K, Kane, B, Kohnen, W: Locally harmonic Maass forms and the kernel of the Shintani lift. Int. Math. Res. Not. 2015(11), 3185-3224 (2015)

6. Bruinier, JH: Borcherds products on $O(2, l)$ and Chern classes of Heegner divisors, Lecture Notes in Mathematics 1780, p. 154. Springer-Verlag, Berlin, Heidelberg, New York, (2002)

7. Bruinier, JH, Funke, J: On two geometric theta lifts. Duke Math J. 125(1), 45-90 (2004)

8. Bruinier, JH, Ono, K: Heeger divisors, L-Functions, and harmonic weak maass forms. Ann. Math. 172, 2135-2181 (2010)

9. Bruinier, JH, van der Geer, G, Harder, G, Zagier, D: The 1-2-3 of Modular Forms, p. 266. Springer-Verlag, Berlin, Heidelberg, (2008)

10. Brylinski, J-L: Heights for local systems on curves. Duke Math. J. 105(1), 1-26 (1989)

11. Cohen, $\mathrm{H}$ : Sums Involving the Values at Negative Integers of L-Functions of Quadratic Characters. Math. Ann. 217 171-185 (1975)

12. Eichler, M, Zagier, D: The theory of Jacobi forms. Progress in Mathematics, vol. 55, Birkhäuser, Boston, Basel, Stuttgart (1985)

13. Funke, J, Millson, JJ: Cycles with local coefficients for orthogonal groups and vector-valued Siegel modular forms. Am. J. Math. 128, 899-948 (2006)

14. Funke, J, Millson, JJ: Spectacle cycles with coefficients and modular forms of half-integral weight, in Arithmetic Geometry and Automorphic Forms, in honor of Stephen S. Kudla. Higher Eduction Press and International Press (2011)

15. Gross, B, Kohnen, W, Zagier, D: Heegner points and derivatives of L-Series, II. Math. Ann. 278(1-4), 497-562 (1987)

16. Hirzebruch, F, Zagier, D: Intersection numbers of curves on Hilbert modular surfaces and modular forms of Nebentypus. Invent. Math. 36, 57-114 (1976)

17. Hopkins, K: Higher weight Heegner points. Exp. Math. 19(3), 257-266 (2010)

18. Kohnen, W: Modular forms of half-integral weight on $\Gamma_{0}(4)$. Math. Ann. 248, 249-266 (1980)

19. McGraw, WJ: Rationality of vector valued modular forms associated to the weil representation. Math. Annalen. $\mathbf{3 2 6}$ 105-122 (2003) 
20. Milne, J: Abelian Varieties (v2.00), 166+vi (2008). Available on www.jmilne.org/math/

21. Nakajima, S: On invariant differential operators on bounded symmetric domains of type IV. Proc. Japan Acad. 58(Ser. A), 235-238 (1982)

22. Scheithauer, NR: The Weil representation of $S L_{2}(\mathbb{Z})$ and some applications. Int. Math. Res. Not. 2009(8), 1488-1545 (2009)

23. Schoen, C: Complex multiplication cycles on elliptic modular threefolds. Duke Math. J. 53, 771-794 (1986)

24. Scholl, AJ: Fourier coefficients of Eisenstein series on non-congruence subgroups. Math. Proc. Camb. Phil. Soc. 99, $11-17(1986)$

25. Shintani, T: On Construction of Holomorphic Cusp Forms of Half Integral Weight. Nagoya Math. J. 58, 83-126 (1975)

26. Strömberg, F: Weil Representations Associated to Finite Quadratic Modules. Math. Z. 275(1), 509-527 (2013)

27. Vignéras, MF: Arithmétique des Algèbres des Quaternions, Lecture Note in Math. 800 (1980)

28. Zemel, S: A $p$-adic approach to the weil representation of discriminant forms arising from even lattices. Math. Ann. Québec. 39(1), 61-89 (2015)

29. Zemel, S: Weight changing operators for automorphic forms on grassmannians and differential properties of certain theta lifts, submitted for publication. a.i. http://arxiv.org/abs/1409.0793

30. Zemel, S: Regularized Pairings of Meromorphic Modular Forms and Theta Lifts, submitted for publication. a.i. http://arxiv.org/abs/1409.0793

31. Zhang, S: Heights of Heegner Cycles and Derivatives of L-Series. Invent. Math. 130(1), 99-152 (1997)

Submit your manuscript to a SpringerOpen ${ }^{\circ}$ journal and benefit from:

$\checkmark$ Convenient online submission

- Rigorous peer review

- Immediate publication on acceptance

- Open access: articles freely available online

- High visibility within the field

Retaining the copyright to your article

Submit your next manuscript at $\boldsymbol{\triangleright}$ springeropen.com 